\title{
Synthesis, structure and applications of graphene-based 2D heterostructures
}

DOI:

10.1039/C7CS00160F

\section{Document Version}

Accepted author manuscript

Link to publication record in Manchester Research Explorer

\section{Citation for published version (APA):}

Solís-Fernández, P., Bissett, M., \& Ago, H. (2017). Synthesis, structure and applications of graphene-based 2D heterostructures. Chemical Society Reviews, 46, 4572-4613. https://doi.org/10.1039/C7CS00160F

\section{Published in:}

Chemical Society Reviews

\section{Citing this paper}

Please note that where the full-text provided on Manchester Research Explorer is the Author Accepted Manuscript or Proof version this may differ from the final Published version. If citing, it is advised that you check and use the publisher's definitive version.

\section{General rights}

Copyright and moral rights for the publications made accessible in the Research Explorer are retained by the authors and/or other copyright owners and it is a condition of accessing publications that users recognise and abide by the legal requirements associated with these rights.

\section{Takedown policy}

If you believe that this document breaches copyright please refer to the University of Manchester's Takedown Procedures [http://man.ac.uk/04Y6Bo] or contact uml.scholarlycommunications@manchester.ac.uk providing relevant details, so we can investigate your claim.

\section{OPEN ACCESS}




\section{Chem Soc Rev}

\section{Synthesis, structure and applications of graphene-based 2D heterostructures}

\begin{tabular}{|r|l|}
\hline Journal: & Chemical Society Reviews \\
\hline Manuscript ID & CS-REV-03-2017-000160.R2 \\
\hline Article Type: & Review Article \\
\hline Date Submitted by the Author: & $20-J u n-2017$ \\
\hline Complete List of Authors: & $\begin{array}{l}\text { Solís-Fernández, Pablo; Kyushu University, Global Innovation Center } \\
\text { Bissett, Mark; University of Manchester, School of Materials } \\
\text { Ago, Hiroki; Kyushu University, Global Innovation Center }\end{array}$ \\
\hline \multicolumn{2}{l}{} \\
\hline
\end{tabular}

\section{SCHOLARONE ${ }^{\text {TH }}$ \\ Manuscripts}




\title{
Journal Name
}

\section{ARTICLE}

\section{Synthesis, structure and applications of graphene-based 2D heterostructures}

Received 00th January 20xx, Accepted 00th January 20xx

DOI: $10.1039 / x 0 x \times 00000 x$ www.rsc.org/

\begin{abstract}
Pablo Solís-Fernández, ${ }^{a}$ Mark Bissett ${ }^{\mathrm{b}}$ and Hiroki Ago* ${ }^{\mathrm{a}, \mathrm{c}, \mathrm{d}}$
With the profuse amount of discovered two-dimensional (2D) materials and the improvements in their synthesis and handling, the field of 2D heterostructures is gaining an increased interest in the recent years. Such heterostructures not only overcome the inherent limitations of each of the materials, but also allow to realize novel properties by their proper combination. The physical and mechanical properties of graphene confer it a prominent place in the area of $2 \mathrm{D}$ heterostructures. In this review, we will discuss the evolution and current state in the synthesis and applications of graphene-based 2D heterostructures. In addition to stacked and in-plane heterostructures with other 2D materials and their potential applications, we will also cover heterostructures realized with lower dimensionality materials, along with intercalation in few-layer graphene as a special case of heterostructure. Finally, graphene heterostructures produced by liquid phase exfoliation techniques and their applications to energy storage will be reviewed.
\end{abstract}

\section{General introduction}

Graphene is an atomically thin single layer of carbon atoms covalently bound in a honeycomb lattice, and the basic building block of graphite. This structure confers to graphene some exceptional characteristics, including its mechanical strength and flexibility, high charge carrier mobility, high optical transparency and a work function that can be tuned by electrostatic or chemical doping. ${ }^{1}$ However, it was not until 2004 when a method was developed to exfoliate graphite into single graphene layers by simply using adhesive tape. ${ }^{2}$ Although obtained by this simple method, this work demonstrated changes in the resistivity by a factor of 100 by electrostatic doping at low temperatures, and carrier mobilities up to $10^{4} \mathrm{~cm}^{2} \mathrm{~V}^{-1} \mathrm{~s}^{-1}$. Within a few weeks, another group independently reported the growth of ultrathin graphite films on SiC, providing a method for the growth of graphene at large scales. ${ }^{3}$ These groundbreaking works set the focus on graphene, the first 2D material ever obtained. Very soon other elaborate methods were developed to produce graphene. These include the already mentioned epitaxial growth on $\mathrm{SiC}$; chemical vapor deposition (CVD) on several metal catalysts using a wide range of gaseous, liquid and solid precursors; ${ }^{4}$ exfoliation and reduction of graphite oxide $;^{5}$ and several liquid phase exfoliation techniques. ${ }^{6}$

However, the limitations of graphene soon became evident.

\footnotetext{
a. Global Innovation Center (GIC), Kyushu University, Fukuoka 816-8580, Japan.

E-mail: h-ago@gic.kyushu-u.ac.jp

${ }^{b .}$ National Graphene Institute, School of Materials, University of Manchester, Manchester M13-9PL, United Kingdom.

c. Interdisciplinary Graduate School of Engineering Sciences, Kyushu University, Fukuoka 816-8580, Japan.

d. PRESTO, Japan Science and Technology Agency (JST), Saitama 332-0012, Japan.
}

Among these, probably the most important issue is the lack of a bandgap in its electronic structure. ${ }^{2}$ This has prevented the efficient use of graphene for several electronic and optoelectronic applications. As a consequence of these limitations, interest in new families of 2D layered materials has emerged in recent years. ${ }^{7}$ The most remarkable of these, given their technological importance, is hexagonal boron nitride ( $h-$ $\mathrm{BN}),{ }^{7,8}$ a layered insulator with a structure similar to that of graphene, as well as the family of transition metal dichalcogenides (TMDs) from the group $\mathrm{VI}^{7,9}$ which are semiconductors with bandgaps between 1 and $2 \mathrm{eV}$. Interestingly, 2D heterostructures can be made by combining graphene with one or more of these materials, as illustrated in Figure 1 . Such heterostructures not only serve to overcome the inherent limitations of each of the materials, ${ }^{10}$ but novel properties can be realized by their proper combination. For the sake of brevity, from now on the term heterostructure in this text will refer to a heterostructure of graphene with other materials, unless otherwise noted.

In this review, we will describe the evolution and the current state in the synthesis and applications of graphene-based heterostructures. After a brief introduction in section 2 to some of the already synthesized 2D materials, section 3 will cover the existing methods to synthesize thin-film heterostructures of graphene with other 2D materials, along with their properties and potential applications. Heterostructures with lower dimension materials and those obtained by intercalation in bi- to few-layer graphene will also be addressed in this section. Section 4 will be devoted to heterostructures processed in liquid solutions, which we will denote hybrid composites. As will be shown, the importance of liquid processed composites resides in the large throughput obtained, along with their convenience for applications related 
with energy production and storage.

\section{Brief introduction to non-graphene 2D materials}

As general overviews to graphene can be found in different articles of this volume, here we will briefly introduce only other 2D materials that are commonly employed in heterostructures with graphene. Less than one year after their groundbreaking work on the mechanical exfoliation of singlelayer graphene, ${ }^{2}$ the same group reported the stability of many other layered 2D crystals at room temperature. ${ }^{7}$ Some of the materials thinned down to single or few layers in this pioneer work included $h-\mathrm{BN}$, some TMDs and $\mathrm{Bi}_{2} \mathrm{Sr}_{2} \mathrm{CaCu}_{2} \mathrm{O}_{\mathrm{x}}$, a high temperature bulk superconductor. As in the case of graphene, the obtained 2D crystals are the building blocks of layered materials held together by weak van der Waals interactions. The methods to obtain isolated single layers have greatly evolved since these first attempts, both for top-down exfoliation techniques and for bottom-up approaches. Currently there exist a wide range of techniques to produce 2D materials, the choice of which depends on the specific material, and on the required applications. The most common synthesis methods include bottom-up approaches, such as CVD, chemical vapor transport (CVT) or other physical deposition methods (molecular beam epitaxy (MBE), magnetron sputtering or physical vapor deposition); $;^{11,12}$ and colloidal growth. ${ }^{13}$ Top-down approaches other than mechanical exfoliation are also widely extended, commonly involving exfoliation in some liquid medium and sometimes assisted with chemical reactions. ${ }^{14}$ Interestingly, most of these mentioned methods can be adapted to the production of heterostructures with graphene.

Simultaneous to the evolution of synthesis techniques, the amount of discovered and produced 2D materials have significantly increased during the last decade, ${ }^{14-16}$ with the new materials covering an extensive spectrum of electronic properties that vastly expand the potential applications from those expected for the original graphene. Among the family of 2D materials there are insulators, such as $\mathrm{h}-\mathrm{BN} ;^{8}$ semiconductors covering a wide range of band gaps, such as $\mathrm{MoS}_{2}{ }^{9}$ or phosphorene; ${ }^{17}$ zero-gap semiconductors such as graphene; metals, such as $\mathrm{NbS}_{2}{ }^{18}$ or the unstable $1 \mathrm{~T}$ phase of some TMDs; ${ }^{19}$ and superconductors, such as $\mathrm{NbSe}_{2},{ }^{20} \mathrm{FeSe}^{21}$ or $\mathrm{Bi}_{2} \mathrm{Sr}_{2} \mathrm{CaCu}_{2} \mathrm{O}_{8+\times \cdot}{ }^{22}$ Molecular nanosheets are also an emerging new class of $2 \mathrm{D}$ materials with a great potential in heterostructures, ${ }^{23,24}$ which will be briefly introduced in section 3.2.5 of this review. Structures of some of the most representative 2D materials are shown in Figure 2. Among the different $2 \mathrm{D}$ materials, there are truly atomically thin ones, as is the case of graphene or $h-\mathrm{BN}$, and those whose structure comprises a few atomic layers, such as the case of TMDs, or with a crumpled structure, as in the case of phosphorene. However, in either case the estimated thickness is below or around $1 \mathrm{~nm}$. Although non-layered 2D materials, such as $\mathrm{WO}_{3}, \mathrm{PbS}$ or ultrathin metal films, are out of the scope of this review, recently they have been attracting an increasing interest for their wide range of applications. ${ }^{25}$

Owing to their technological importance, $h-\mathrm{BN}$ and the family of TMDs are among the most prominent and studied layered materials. Boron nitride can present several crystalline forms, the most stable being the hexagonal boron nitride, often abbreviated as $h-\mathrm{BN}$. $h-\mathrm{BN}$ possess a hexagonal lattice analogous to that of graphene, although with boron and nitrogen atoms respectively occupying the two inequivalent sublattices (Figure $2 \mathrm{~b}$ ). Owing to the ionic nature of the bonding of the $\mathrm{B}$ and $\mathrm{N}$ atoms, $h-\mathrm{BN}$ is an insulator with a large band gap of $\sim 5.97 \mathrm{eV}$, in contrast with the gapless nature of graphene. The lattice constants of graphene and $h-$ $\mathrm{BN}$ are also quite similar, with a lattice mismatch of only $1.8 \%$. As we shall see in the following sections, this mismatch induces some interesting changes in the electronic structure of graphene when it is in the proximity of $h-\mathrm{BN}$.

The family of the TMDs present the general form $\mathrm{MX}_{2}$, where $M$ is a transition metal, commonly from the groups IV-VI, such as $W$ and $M o$, and $X$ is an element from the group of the chalcogens, usually $S$ and $S e$, and to a less amount Te. These materials are composed of a 3-atom layer of the form $\mathrm{X}-\mathrm{M}-\mathrm{X}$, with a plane of hexagonally packed metal atoms separating two planes of chalcogen atoms. The bonds formed between the metal and the chalcogen are predominantly covalent. Depending on the geometrical coordination of the metal atoms, the structures of the TMD present two different polymorphs known as $2 \mathrm{H}$ (trigonal prismatic) or $1 \mathrm{~T}$ (octahedral). The electronic properties of TMDs cover a wide range from metals to insulators. From here we will mostly focus on the group VI of TMDs $\left(\mathrm{MoS}_{2}, \mathrm{WS}_{2}, \mathrm{MoSe}_{2}\right.$, and WSe $\mathrm{WS}_{2}$ ) for which the $2 \mathrm{H}$ is the thermodynamically stable coordination (Figure 2c). ${ }^{26}$ In the $2 \mathrm{H}$ configuration these TMDs are semiconductors, covering a range of gaps between 1 and $2 \mathrm{eV}$, while they are metallic in the less stable $1 \mathrm{~T}$ polymorph. Although the bandgap is indirect in the case of the bulk materials, it becomes a direct when they are thinned down to single layers. The direct bandgap, along with their high absorption coefficients $\left(>10^{7} \mathrm{~m}^{-1}\right),{ }^{27}$ make TMDs interesting materials for optoelectronic applications.

\section{Graphene-based heterostructures}

\subsection{Thin film graphene heterostructures}

As mentioned in the introduction, the extraordinary properties of graphene are not exempt of some drawbacks, in most cases related with the absence of a bandgap. ${ }^{2}$ Considering the wide range of existing 2D materials, and with some of them exhibiting complementary properties, their integration into heterostructures is interesting and promising. ${ }^{10}$ Heterostructures can vastly increase the potential of such materials, bringing unique functionalities for such thin materials. The properties of the different materials in the heterostructures can be combined to produce all-2D devices with new and interesting properties and applications, including novel electronic and optoelectronic devices. ${ }^{28}$ Among the most 
significant properties that graphene can contribute for heterostructures are its high conductivity and carrier mobility, along with its transparency and mechanical flexibility. ${ }^{29}$

Given the layered nature of most of the 2D materials, the most evident and feasible way to construct such heterostructures is by artificially stacking layers of the different materials. ${ }^{10,28}$ Van der Waals 2D layered materials present a high anisotropy between the in-plane and out-of-plane directions, with strong covalent bonds within the layer but weak van der Waals interactions between the layers. As already seen, this weak inter-layer interaction is essential to isolate single layers by exfoliation, ${ }^{7}$ but also permits the stacking of completely different materials without restrictions arising from the mismatch in their crystal lattices. ${ }^{28}$ This allows the fabrication of virtually any kind of vertical heterostructures by mechanically piling up the different layers in the desired sequence. ${ }^{10}$ As we will see in the following sections, the fast advance in the processing of $2 \mathrm{D}$ materials has provided an easy way to produce quite complex vertical heterostructures with diverse functionalities. Routes to synthesize such heterostructures by avoiding mechanical stacking are being pursued at the same time, in order to achieve high throughputs required for their mass production. ${ }^{28}$

Lateral, in-plane heterostructures in which one-dimensional junctions are created between the different materials (Figure 1 ), are also interesting. ${ }^{30-32}$ This kind of heterostructures opens the possibility to obtain ultimately thin devices. However, the development in this case has been slower than for vertical heterostructures. There are two main reasons for this. The first and foremost is the lack of a straightforward method to produce such lateral heterostructures, similar to that of mechanical stacking for vertical heterostructures. Thus, all of the existing methods rely on the bottom-up synthesis of at least one of the materials. The second reason arises from the necessity to realize in-situ processing techniques, in which one of the materials starts to grow from the edge of the other. This can result in damage of the original material, requiring the development of synthesis techniques compatible for all the involved materials. Nonetheless, as will be seen in the corresponding section, some interesting advances have been realized in the last years and a few in-plane graphene-based heterostructures have being reported. Although in most cases the heterostructures present a small overlap in the junction, some cases of truly 1D stitching have been observed at the interface between graphene and $h-\mathrm{BN}^{33-35}$

\subsection{Stacked van der Waals graphene heterostructures}

\subsubsection{Synthesis methods: mechanical stacking vs. direct growth}

Mechanical stacking of 2D layered materials was first demonstrated in 2010 by Dean et al. for the production of graphene FET devices supported by $h-\mathrm{BN} .^{8}$ The process started with the mechanical exfoliation of a thin film of $h-\mathrm{BN}$ onto the target substrate, along with the exfoliation of the graphene on a PMMA thin membrane. Graphene was then carefully aligned with the $h-\mathrm{BN}$, and both were brought into contact with the aid of a micromanipulator (Figure 3a). By repeating this procedure it is possible to stack an arbitrary number of additional layers of different materials, ${ }^{36-41}$ thus increasing the complexity and the functionality of the potential heterostructures that can be assembled (Figure 3b). However, this method is likely to introduce some contamination to the interface of the stacked layers, due to the processes involved during the transfer of the different layers. ${ }^{42}$ The presence of these contaminants has a negative effect on the quality of the heterostructure. ${ }^{41}$ Almost pristine interfaces could be realized after annealing the heterostructure at moderate temperatures $\left(300^{\circ} \mathrm{C}\right)$, which induces the aggregation of most of the contaminants into small delimited regions of the interface. ${ }^{41,43}$ However, even these low temperatures may result in permanent damage for certain heterostructures. ${ }^{44}$ The methods for mechanically stacking 2D materials have evolved through the years by exploiting the van der Waals interactions existing between them, resulting in different dry transfer methods. In dry transfer methods the first exfoliated flake is employed to progressively pick up the rest of the exfoliated layers that will conform the final heterostructures (Figure 3c). ${ }^{44-47}$ This method results in cleaner interfaces between the stacked layers by avoiding a direct contact of the interface surfaces to polymers or solvents. It should still be noted that these surfaces are in general exposed to ambient conditions during the exfoliation and stacking, leading to the unavoidable presence of water, hydrocarbons and other kinds of contamination. ${ }^{48}$ Transfer in inert atmosphere can however be realized in the case of materials unstable in air, such as $\mathrm{NbSe}_{2}{ }^{49}$ Currently employed stack techniques allow the realization of highly oriented heterostructures with misalignments of the lattices below $2^{\circ}$, with enough precision to fabricate artificial stacks of Bernal-stacked bilayer graphene. ${ }^{50}$ Moreover, van der Waals interactions between the layers can also assist to increase the degree of alignment of the heterostructures, by adjusting the relative angle to the most stable configuration. ${ }^{51}$ In this sense, recent works have demonstrated the self-alignment of large flakes of drytransferred graphene on $h-\mathrm{BN}$ by annealing at temperatures over $100{ }^{\circ} \mathrm{C}$ in $\mathrm{Ar}$ or $\mathrm{Ar} / \mathrm{H}_{2}$ (Figure 3d). ${ }^{51,52}$ The misalign angle ends up being below $\sim 0.7^{\circ}$ after the annealing, producing highly aligned heterostructures. ${ }^{51}$ Although $0^{\circ}$ is the most stable configuration, flakes initially twisted by $\sim 20^{\circ}$ tend to rotate to $30^{\circ}$ during the annealing. ${ }^{52}$ The thermal selfalignment is driven by the minimization of interaction energy between the layers, so it is expected to be also present for other different layers, and in fact has been successfully tested for graphene-on-graphene stacks. ${ }^{51,53}$ Solely relying on the van der Waals interaction between the different 2D layers, the drystacking methods can result in a low transfer yield. The transfer process can be improved by exploiting the different thermal coefficients of the polymers employed for the transfer, and those of the $2 \mathrm{D}$ layers. ${ }^{43}$ Recently, a new technique has been developed to exfoliate and transfer largearea single-layer TMDs onto several substrates. ${ }^{54} \mathrm{~A}$ thin film of $\mathrm{Au}$ is evaporated on top of the bulk TMD and then peeled with a thermal release tape. The large affinity between the Au film and the chalcogen ensures the peeling of only the top layer of 
the TMD, allowing to obtain large, isolated single layers with lateral sizes up to a few hundred microns. The optical and electronic quality of these samples rival those obtained by other exfoliation techniques, while the size and homogeneity obtained are far superior. ${ }^{54}$

The use of layers exfoliated from the bulk materials make the mechanical stacking methods appropriate for fast prototyping of layered heterostructures without the need to develop methods to directly grow $2 \mathrm{D}$ versions of the materials. Another advantage of these approaches is that a lattice match between the two materials forming the interface is not required, and thus completely different materials can be piled up together without any restriction. Moreover, control of the relative rotation between the different layers can be attained with a high precision, ${ }^{50}$ allowing the fine tuning of the properties of the heterostructures. Consequently, these methods have been widely used for fundamental studies to produce a vast amount of different heterostructures, such as several kinds of TMDs with graphene, including $\mathrm{MoS}_{2}{ }^{44,55-57,27,58-62}$ and $\mathrm{WS}_{2}{ }^{44,27,63}$ the mentioned case of $h-\mathrm{BN}$ and graphene; $8,44,36-38,40,41,45,63-67$ mixed heterostructures containing graphene, $h-\mathrm{BN}$ and TMDs; ${ }^{65,66}$ and graphene heterostructures with other materials. $^{44,57}$

Although quite convenient from the point of view of basic research, mechanical stacking methods are not yet suitable for production of graphene heterostructures at industrial scale. Efficient fabrication methods that can be easily scaled-up to mass production are thus required. Methods for the direct growth of graphene heterostructures have been developed, aimed to increase the productivity. At the same time, direct growth methods can provide much cleaner interfaces than those obtained in mechanical stacked samples, especially when two-step growth approaches are employed. Direct bottom-up growth methods have already been reported for the synthesis of $h-\mathrm{BN}$ on top of graphene ${ }^{68,69}$ and of graphene on $h-\mathrm{BN} ;{ }^{70-84}$ of several TMDs on graphene, including $\mathrm{MoS}_{2},{ }^{69,85-96} \mathrm{WS}_{2},{ }^{97-100} \mathrm{MoSe}_{2}{ }^{101}{ }^{101} \mathrm{WSe}_{2}{ }^{69,90,102,103} \mathrm{NbS}_{2}{ }^{18}$ and $\mathrm{NbSe}_{2} ;{ }^{104}$ of successive stacks of different TMDs on graphene; ${ }^{105}$ and of other materials such as GaSe, ${ }^{106,107}$ nontransition metal chalcogenides ${ }^{108-110}$, single layers of metal oxides such as $\mathrm{ZnO},{ }^{111}$ or perovskites ${ }^{112}$ on graphene. Most of those methods to synthesize heterostructures have evolved along with the possibility to synthesize isolated layers of the respective materials by CVD or related methods. In the following sections, some of these methods will be covered in more detail.

\subsection{2. h-BN/graphene heterostructures}

Apart from methods based on mechanical stacking, several procedures have been developed for the growth of $h-\mathrm{BN}$ on graphene. A few methods have been reported for the direct synthesis of $h-\mathrm{BN}$ on graphene. ${ }^{68,69}$ Liu et al. reported the twostep synthesis of $h-\mathrm{BN}$ on graphene. ${ }^{68}$ First graphene is grown on a $\mathrm{Cu}$ foil by $\mathrm{CVD}$ at $950{ }^{\circ} \mathrm{C}$ using hexane as the carbon feedstock. The $h-\mathrm{BN}$ was then grown on the graphene by a second CVD with ammonia borane as the precursor. The thermal decomposition of the ammonia borane at $1000{ }^{\circ} \mathrm{C}$ results in a film of $h-\mathrm{BN}$ almost homogeneously covering the graphene. However, Raman spectroscopy reveals that graphene is damaged during the second CVD.

The synthesis of graphene on $h-\mathrm{BN}$ is more widely studied than the opposite, as the $h-\mathrm{BN}$ is better able to resist the second CVD growth compared to graphene, and such a structure is more useful from the point of view of applications. ${ }^{70-74,76-84}$ In early attempts to grow single-layer graphene/ $h-\mathrm{BN}$ heterostructures, graphene was grown on an epitaxial $h-\mathrm{BN}$ film on $\mathrm{Ni}(111)$ by exposure to large amounts of benzene at $800{ }^{\circ} \mathrm{C}^{70}$ More recent works show the CVD growth of multilayer graphene on $h-\mathrm{BN}$ flakes $^{71}$ and of single-layer graphene on CVD-grown $h-\mathrm{BN},{ }^{74,75}$ both conducted at $1000^{\circ} \mathrm{C}$ by using $\mathrm{CH}_{4}$ as the carbon feedstock (Figure $4 a, b$ ). The growth of graphene by CVD using $\mathrm{CH}_{4}$ usually requires a catalytic surface to decompose the $\mathrm{CH}_{4}$ molecules, with the growth being self-limiting as the graphene starts to cover the catalyst. The mechanisms for the growth of graphene on $h-\mathrm{BN}$ are not completely clear yet, but most probably involve some catalytic transparency of thin $h-\mathrm{BN}$ layers when supported on a surface such as $\mathrm{Cu}^{75,84}$ Even though, the growth procedures should require certain adjustments with respect to the growth on catalytic substrates, such as increases in the feedstock partial pressures, processing temperatures and growth times. ${ }^{77}$ Molecular beam epitaxy (MBE) growth of graphene on exfoliated $h-\mathrm{BN}$ has also been demonstrated at temperatures of $930{ }^{\circ} \mathrm{C}$, although the quality of the graphene is not as good as in the case of CVD methods. ${ }^{72}$ The temperature of the CVD synthesis can be decreased with plasma enhanced CVD (PECVD), which also makes unnecessary the use of a catalyst to decompose the $\mathrm{CH}_{4}$ molecules. The use of PE-CVD allows the growth of graphene on exfoliated $h-\mathrm{BN}$ using $\mathrm{CH}_{4}$ as feedstock at temperatures as low as $500{ }^{\circ} \mathrm{C} .{ }^{78}$ However, these procedures usually result in small graphene grains with sizes well below $1 \mu \mathrm{m}$. Graphene can also be synthesized on $h-\mathrm{BN}$ by LPCVD using a liquid precursors. ${ }^{77,83}$ By using $h-$ $\mathrm{BN} / \mathrm{Cu}(111)^{77}$ and $h-\mathrm{BN} / \mathrm{Rh}(111)^{83}$ as the growth substrates, Roth et al. demonstrated that graphene can be grown with 3pentanone at $\sim 830{ }^{\circ} \mathrm{C}$ and $\sim 880^{\circ} \mathrm{C}$ respectively by LPCVD. In the case of $h-\mathrm{BN} / \mathrm{Rh}(111)$, a two-step LPCVD procedure was used to first decouple the $h-\mathrm{BN}$ from the substrate, resulting in a flatter surface. ${ }^{83}$ In both cases, the graphene is completely aligned with the underlying $h-\mathrm{BN}$, even though the lattice mismatch existing between them ( $1.8 \%$ ) (Figure $4 \mathrm{c}, \mathrm{d}){ }^{77,83}$ Graphene/ $h-\mathrm{BN}$ heterostructures have also been obtained by the confined growth of graphene in the space between $\mathrm{Ni}(111)$ and a CVD-grown disoriented $h-\mathrm{BN}$ layer (Figure $4 \mathrm{e}){ }^{82}$ The growth of the graphene in this case is done by LPCVD at a relatively low temperature $\left(540{ }^{\circ} \mathrm{C}\right)$, using ethylene $\left(\mathrm{C}_{2} \mathrm{H}_{4}\right)$ as the carbon feedstock. Song et al. have recently developed a procedure for the growth of patterned graphene on $h-\mathrm{BN}$ by using PMMA seeds as nucleation points for the graphene and benzoic acid as the precursor (Figure $4 \mathrm{f}-\mathrm{h}$ ). ${ }^{84}$ The growth of patterned heterostructures can be interesting for upscaling the growth of 2D-based electronic circuits.

The use of $h-\mathrm{BN}$ as a substrate for graphene originates from 
the limitations arising from using commonly employed substrates, such as $\mathrm{SiO}_{2}$. These limitations include the surface roughness of the substrate, the presence of charge traps and surface optical phonons, and other inhomogeneities. ${ }^{8,113,114}$ All of these have a negative impact on the performance of graphene-based electronic devices. The benefits of $h-\mathrm{BN}$ as a substrate compared to $\mathrm{SiO}_{2}$ include its chemical inertness, and its ultra-flat surface free from dangling bonds and of charge impurities. ${ }^{8}$ Recent LEEM based studies have determined that for misoriented lattices there is no electronic coupling between graphene and $h-\mathrm{BN} .{ }^{115}$ Thus, the band structure of graphene in the proximity of the Dirac point is not perturbed. As already mentioned, mechanically exfoliated $h-\mathrm{BN}^{7}$ was first employed as a substrate for graphene in $2010 .{ }^{8}$ Graphene on $h-\mathrm{BN}$ has proved to be much flatter than on $\mathrm{SiO}_{2}$ (Figure $5 \mathrm{a}$ ), with a surface roughness similar to those measured for graphene on other atomically flat substrates such as mica or of freshly cleaved graphite, and without surface charge inhomogeneities (see Figure $5 b, c) .^{8,113,116}$ This is accompanied by an improvement of the electronic properties of graphene, with the mobility increasing roughly an order of magnitude and almost no doping induced from the substrate, as shown in (Figure 5d). 8,64,116-118 Taking a step forward, the electronic properties of graphene can be further improved by encapsulating it between $h$-BN layers, attaining mobilities over $10^{5} \mathrm{~cm}^{2} \mathrm{~V}^{-1} \mathrm{~s}^{-1}$ at room temperature. ${ }^{36,119}$ It should be noted that the mobilities achieved on $\mathrm{SiO}_{2}$ rarely exceed values of $2 \times$ $10^{4} \mathrm{~cm}^{2} \mathrm{~V}^{-1} \mathrm{~s}^{-1} \cdot 2,8$ By optimizing the transfer process of the layers, mobilities up to $1.5 \times 10^{5} \mathrm{~cm}^{2} \mathrm{~V}^{-1} \mathrm{~s}^{-1}$ have been realized on exfoliated graphene at room temperature, and of $\sim 5 \times 10^{5}$ $\mathrm{cm}^{2} \mathrm{~V}^{-1} \mathrm{~s}^{-1}$ at temperatures below $20 \mathrm{~K}^{44}$ In the case of CVD graphene, carrier mobilities have been reported as large as 3.5 $\times 10^{5} \mathrm{~cm}^{2} \mathrm{~V}^{-1} \mathrm{~s}^{-1}(1.6 \mathrm{~K})$, and of $3 \times 10^{6}(1.8 \mathrm{~K})$ after optimization of the transfer. ${ }^{67}$ Graphene encapsulated in $h-\mathrm{BN}$ layers shows ballistic transport at micrometer scale, for distances of $\sim 1 \mu \mathrm{m}$ at $200 \mathrm{~K}$ and of more than $28 \mu \mathrm{m}$ at temperatures below $2 \mathrm{~K}^{36,44,45,67}$ Furthermore, encapsulation with $h$-BN layers protects the graphene against the environment. Thus, devices show similar behavior in air and in vacuum, ${ }^{119}$ while the chemical stability of $h-\mathrm{BN}$ preserves the integrity of graphene under extreme environments, such as oxidizing atmospheres at high temperatures. ${ }^{120}$ Encapsulation with $2 \mathrm{D}$ materials can also be used as a general way to protect other 2D materials in harsh conditions, as shown in the decreased damage in graphene-encapsulated 2D materials exposed to the high energy electron beams of TEM. ${ }^{121,122,23}$ As we will cover in following sections, $h-\mathrm{BN}$ has also proved to be an efficient ultrathin dielectric gate ${ }^{36,123}$ and a capable thin tunnel barrier in the fabrication of vertical 2D heterostructures, with the barrier thickness going down to a single layer. ${ }^{40,124}$

Apart from the aforementioned increase of the carrier mobility of graphene, $h-\mathrm{BN}$ has demonstrated to be an ideal substrate to realize some of the most exotic properties of graphene. These include fractional quantum Hall states and broken symmetries, ${ }^{125-127}$ long-distance spin transport over lengths of $20 \mu \mathrm{m}^{128}$ and quantum spin Hall states, ${ }^{129}$ among many others.
Furthermore, specific effects dependent on the stacking angle arise from the small lattice mismatch between the graphene and the $h-\mathrm{BN}$. The presence of $h-\mathrm{BN}$ induces a periodic potential characterized by a Moiré pattern, which can be imaged by STM and AFM (Figure 6a-c). ${ }^{52,78,130}$ For a relative orientation $(\phi)$ between the $h-\mathrm{BN}$ and the graphene, the wavelength of the formed superlattice is given by $\lambda=$ $\frac{(1+\delta) a}{\left(2(1+\delta)(1-\cos \phi)+\delta^{2}\right)^{1 / 2}}$, where $a$ is the lattice constant of graphene and $\delta$ the lattice mismatch with the $h-\mathrm{BN}^{130}$ The superlattice is oriented by an angle $(\theta)$ with respect to the graphene, given by the expression $\tan \theta=\frac{\sin \phi}{(1+\delta)-\cos \phi}$. As reflected in the expression, $\theta$ depends on the relative orientation of the graphene and the $h-\mathrm{BN}(\phi)$, being $0^{\circ}$ when both materials are oriented. ${ }^{130}$ The presence of this periodic potential can significantly alter the electronic properties of graphene. One of the effects of the small lattice mismatch is the possibility to open a bandgap in the graphene. ${ }^{131}$ Transport measurements indicate that the periodic potential induced by the lattice mismatch of $h-\mathrm{BN}$ and graphene can open a measurable bandgap at the main Dirac point of the latter of $27 \mathrm{meV}$ when both are highly aligned (Figure 6d,e). ${ }^{132}$ The magnitude of such bandgap decreases as the lattices misalign, being absent in highly twisted heterostructures. ${ }^{116,132}$ Recently, an even larger bandgap ( $160 \mathrm{meV}$ ) has been observed by ARPES for aligned graphene on $h-\mathrm{BN} .{ }^{133}$ The bandgap arises from an asymmetry between the two sublattices of graphene when is placed near the $h-\mathrm{BN}^{116}$ However, the lattice mismatch between $h-\mathrm{BN}$ and graphene reinstates the symmetry on a spatial average, and hence no bandgap should be observed. In this sense, a gap has not been observed on other works by STS, ${ }^{116,130}$ or in ARPES for aligned graphene grown on $h-\mathrm{BN} / \mathrm{Cu}(111)^{77}$ and on $h-\mathrm{BN}$ decoupled from $\mathrm{Rh}(111) .^{83}$ The experimental discrepancies probably arise from the strain-induced increase of the level of commensuration observed for small misalignment angles, which leads to a global disappearance of the symmetry on the graphene sublattices. ${ }^{134}$ These commensurate areas have been observed to disappear when graphene is encapsulated in $h-\mathrm{BN}$, leading to the vanishing of the bandgap (see Figure $6 f$ ). ${ }^{134}$ On the other hand, recent experiments have shown that the commensuration level can be increased by low temperature annealing in $\mathrm{Ar} / \mathrm{H}_{2}$ due to an increase of alignment. ${ }^{51}$

Another effect of the superlattice periodic potential caused by the $h-\mathrm{BN}$ is the emergence of different sets of Dirac cones. A set of first-generation Dirac cones (FDC) appears at the same energy level of the original Dirac cone in the center of the six nearest superlattice Brillouin zones (SBZ). These have been reported by ARPES both on aligned and misaligned graphene/ $h-\mathrm{BN} .^{133,135} \mathrm{~A}$ set of secondary Dirac cones (SDC) also appear in highly aligned heterostructures on the edges of the SBZ, both on the valence and conduction bands of graphene (Figure 6e). The presence of the SDC have been observed by STS (Figure 6g), ${ }^{130}$ transport measurements $^{52,78,132,136}$ and ARPES ${ }^{133}$. The energy difference of the SDCs with respect to the original Dirac cone increases with the misalignment of the graphene and $h-\mathrm{BN}$ (Figure 
$6 \mathrm{~g}, \mathrm{~h}) .{ }^{52,130}$ This, along with weaker interaction between graphene and $h-\mathrm{BN}$ at large misorientation angles makes it difficult to observe the SDCs for misaligned samples. ${ }^{135}$ ARPES measurements have determined the existence of a bandgap of $\sim 100 \mathrm{meV}$ for the SDCs in highly aligned heterostructures, similar to that observed for the original Dirac cone (Figure $6 i, j) .{ }^{133}$ The modification of the band structure of graphene with superlattices is interesting from the viewpoint of applications, as it allows the tuning of the electronic properties of the heterostructures. For example, anisotropic graphene heterostructures can be realized, as the additional Dirac points induce an anisotropy in the velocities of the charge carriers in graphene. ${ }^{130}$ The presence of the satellite Dirac points have been observed to cause Fabry-Pérot resonances, indicating the possibility to confine electrons by engineering the periodic potential caused by the $h-\mathrm{BN} .^{137}$

\subsection{3. $\mathrm{TMD} / \mathrm{graphene}$ heterostructures}

Heterostacks of graphene and TMDs can be obtained by the general mechanical stacking methods covered in section 3.2.1..$^{44,55-57,27,58-63}$ Here, we will briefly introduce other synthesis methods and some of the most relevant kind of heterostructures that have been realized up to date. Several TMDs have been successfully grown on graphene. ${ }^{18,69,85-105}$ Apart from the mechanical stacking, the most widespread methods to synthesize graphene/TMD heterostructures rely on the CVD synthesis of the TMD over the graphene. Usual temperatures to grow high-quality graphene by CVD (> 1000 ${ }^{\circ} \mathrm{C}$ ) can damage the TMD. This, along with the need of a metal catalyst surface to grow graphene, makes this approach more prevalent than the growth of the graphene on the TMD. The most studied TMD to date is $\mathrm{MoS}_{2}$, and hence the literature of $\mathrm{MoS}_{2}$ grown on graphene outnumbers that of other TMDs. ${ }^{69,85-96}$ One of the earliest reported attempts to grow few layer $\mathrm{MoS}_{2}$ on graphene was by Shi et al., ${ }^{85}$ using ammonium thiomolybdate $\left(\left(\mathrm{NH}_{4}\right)_{2} \mathrm{MoS}_{4}\right)$ deposited on the graphene (Figure 7a). The growth was done by the sequential decomposition of $\left(\mathrm{NH}_{4}\right)_{2} \mathrm{MoS}_{4}$ at $400{ }^{\circ} \mathrm{C}$ in the presence of hydrogen. However, this approach is complicated and requires several steps. Besides, the control on the number of layers is not assured, while the $\mathrm{MoS}_{2}$ is composed of randomly oriented small crystals. Using copper hexadecafluorophthalocyanine $\left(\mathrm{F}_{16} \mathrm{CuPc}\right)$ as a seed promoter, Ling et al. demonstrated that it is possible to grow $\mathrm{MoS}_{2}$ on exfoliated graphene by APCVD at the relatively low temperature of $650{ }^{\circ} \mathrm{C}$ (Figure $7 \mathrm{~b}$ )..$^{86}$ By using higher temperatures for the graphene substrate $\left(\sim 960^{\circ} \mathrm{C}\right)$, it has been demonstrated the growth of $\mathrm{MoS}_{2}$ on graphene by APCVD without promoters (Figure 7c,d). ${ }^{87,94}$ Given that the graphene is obtained by CVD, it should be noted that large scale heterostructures are also possible. The orientation of the $\mathrm{MoS}_{2}$ coincided in this case with that of the graphene template, indicating heteroepitaxial growth (Figure 7d). This method has also been used on graphene nanoribbons, allowing the growth of nanoribbon heterostacks of $\mathrm{MoS}_{2}$ /graphene with both partial and total $\mathrm{MoS}_{2}$ coverages. ${ }^{88}$ The use of low pressures during the CVD also allows the rotationally commensurate growth of $\mathrm{MoS}_{2}$ on epitaxial graphene (EG) at $800{ }^{\circ} \mathrm{C}$ (Figure 7e,f). ${ }^{93}$ Control of the orientation results essential, as the electronic structure of TMDs/graphene depends on the relative orientation, ${ }^{138}$ as in the case of graphene/h-BN.

For the CVD growth of $\mathrm{MoS}_{2}$ on graphene, the most used precursors are $\mathrm{S}$ and $\mathrm{MoO}_{3}$, although $\mathrm{Cl}$-based compounds can also be used as the Mo feedstock. ${ }^{139} \mathrm{MoO}_{3}$ is located on the hot area of the furnace and usually close to the substrate, while $S$ is placed upstream on a colder area $\left(<200{ }^{\circ} \mathrm{C}\right)$, and both are carried to the growth area by a stream of either Ar or $\mathrm{Ar} / \mathrm{H}_{2}$. Thus, the precursors can be changed for the growth of different TMDs, e.g. using $\mathrm{WO}_{3}$ and Se instead of $\mathrm{MoO}_{3}$ and $\mathrm{S}$ respectively, to obtain $W_{S e_{2}}$. In this way, $W_{2}$ has been epitaxially grown on CVD graphene as well as on EG on SiC using $\mathrm{WO}_{3}$ and $\mathrm{S}$ as precursors (Figure 7g). ${ }^{98,99}$ Kim et al. developed a slightly modified approach in which a thin film of $\mathrm{WO}_{3}$ deposited on $\mathrm{SiO}_{2}$ is put in direct contact with the graphene during the growth process. ${ }^{99}$ By using Se, the CVD growth of $\mathrm{MoSe}_{2}{ }^{101}$ and of $\mathrm{WSe}_{2}{ }^{69,102}$ on graphene has also been demonstrated. Direct growth of a thin film of $\mathrm{NbS}_{2}$ has been also achieved on graphene using $\mathrm{NbCl}_{5}$ and $\mathrm{S}$ as precursors (Figure $7 \mathrm{~h}$ ). ${ }^{18}$ In this case, graphene proved to be a critical factor to favor the lateral growth of the $\mathrm{NbS}_{2}$ nanosheets.

In the previously mentioned works the TMD is grown either on exfoliated graphene or on already synthesized graphene in a different process. It is interesting to grow the heterostructures sequentially, without the need to remove the sample from the processing chamber between the growths of the different materials. In this sense, Shi et al. have reported the production of $\mathrm{MoS}_{2}$-graphene heterostructures in a two-step CVD. ${ }^{91}$ First, graphene is grown on $\mathrm{Au}$ using $\mathrm{CH}_{4}$ at ambient pressure, and then a subsequent step was conducted at low pressure for the growth of $\mathrm{MoS}_{2}$ from $\mathrm{MoO}_{3}$ and S (Figure 8a). However, the $\mathrm{MoS}_{2}$ grains are randomly oriented with respect to the underlying graphene, as opposed to other works (Figure 8b). The two-step CVD growth of heterostructures is still interesting in terms of processing at large scales and to obtain cleaner heterostructure interfaces. Although scarce, other methods apart from CVD have been reported for the growth of TMDs on graphene, such as MBE, ${ }^{92,96}$ sulfurization of deposited metal $^{89,95}$ and metal-organic CVD (MOCVD). ${ }^{103}$

The possibility to control the orientation of TMD grains grown on graphene ${ }^{87,93}$ allows to manipulate and restrict the kind of grain boundaries formed in the TMD $(\mathrm{GBs}) .{ }^{140}$ This results interesting to obtain more reproducible heterostructures, while also opens the opportunity for the tuning of their electronic properties in a similar way as for graphene/ $h$ BN. ${ }^{140,141}$ The oriented growth of TMDs has also been used to visualize the crystal orientation and grain structure of the underlying graphene (Figure $8 \mathrm{c}$ ).$^{94}$ This is done by comparing the relative orientation of $\mathrm{MoS}_{2}$ grains across the surface, which allows the mapping of the crystal structure of the underlying polycrystalline graphene given the existing epitaxial relation (Figure $8 \mathrm{~d}, \mathrm{e}$ ). As the $\mathrm{MoS}_{2}$ nucleation is promoted at the grain boundaries of the graphene, this technique also 
allows their direct visualization.

An improvement in the stability in air of TMDs directly grown on graphene has been observed without the need of encapsulation. ${ }^{99}$ This is attributed to the strong interlayer interaction and to the higher crystallinity of the TMD crystals synthesized on the graphene. On the other hand, an enhancement of the electronic properties of graphene has been observed when using some TMDs as substrate, although the performance is below that attained for $h-\mathrm{BN}$ substrates. ${ }^{59,142}$ Some other reports indicate that the structural defects on the crystal structure of the TMDs can have a negative impact on the electronic properties of the supported graphene. ${ }^{143}$ When enclosed between $h-\mathrm{BN}$ and a TMD (either $\mathrm{MoS}_{2}$ or $\mathrm{WS}_{2}$ ), graphene presented high carrier mobilities up to $6 \times 10^{4} \mathrm{~cm}^{2} \mathrm{~V}^{-1} \mathrm{~s}^{-1}$ (Figure 8f). ${ }^{44}$ However, encapsulation between flat layered oxides (such as mica, BSCCO or $\mathrm{V}_{2} \mathrm{O}_{5}$ ) resulted in graphene mobilities of only $\sim 10^{3} \mathrm{~cm}^{2} \mathrm{~V}^{-1} \mathrm{~s}^{-1}$.

\subsubsection{Applications}

\section{a) Optoelectronics}

Among the many applications of graphene-based heterostructures, the most interesting ones are those in the field of optoelectronics. Optoelectronic devices are electronic devices with the potential to interact with electromagnetic radiation, mainly in the visible region of the spectrum. These devices are mostly used to control, detect or generate light. Graphene can interact with light with a broad range of wavelengths, with a wavelength independent adsorption of $2.3 \%$ of the incident light in the visible and infrared range for a single-layer. ${ }^{29}$ This, along with its high response speed and carrier mobility, and its flexibility, are interesting properties for optoelectronic applications. However, the lack of a bandgap also translates into optoelectronic devices with very poor characteristics due to the short lifetime of the exciton separation. ${ }^{29}$ On the other hand, most TMDs are directbandgap semiconductors when thinned down to single layers, also exhibiting large optical absorptions $\left(>10^{7} \mathrm{~m}^{-1}\right) .^{27}$ Moreover, the interaction of graphene with the TMDs can be used to modify their electronic and optoelectronic properties, $^{144,145}$ which opens new ways to realize optoelectronic devices. In the following sections we will show some recent results of graphene heterostructures with application in optoelectronics. In most of these heterostructrures, the TMD is used as the optical active material.

\section{b) Broadband ultrafast photodetection and light harvesting}

Photodetectors are devices designed to convert optical signals to electric signals. This is generally conducted in three steps, with the light generating charge carriers, which then need to be separated and carried to each electrode as a photocurrent. The efficiency of a photodetector is usually expressed by the external quantum efficiency (EQE) (sometimes expressed as $\eta$ ), a parameter that represents the fraction of incident photons that are converted in photocarriers. Another commonly employed parameter is the responsivity, $R$, a measure of the electrical output per optical input and with units of $A W^{-1}$. The responsivity and the EQE are related by the expression $R=\eta \frac{e}{h v}$, with $e$ being the elementary charge and $h v$ the energy of the incident photons.

Stacks of CVD-grown graphene with TMDs have been extensively studied to produce highly sensitive photodetectors. One of the approaches to make graphene/TMD photodetectors is to use the TMD as a photogate, with the photocurrent flowing through the graphene. In this way, Roy et al. fabricated photodetectors by stacking single-layer graphene on few-layer $\mathrm{MoS}_{2}$ (5 to 10 layers) (Figure 9a)..$^{55}$ The transfer characteristics of the graphene is influenced by the underlying $\mathrm{MoS}_{2}$, which screens the gate for gate voltages $\left(V_{G}\right)$ larger than the $M_{0} S_{2}$ threshold voltage $\left(V_{T}\right)$ (Figure 9a). Upon illumination for $V_{G}<V_{T}$, a gatedependent photocurrent $\left(I_{P}\right)$ is observed in the graphene due to the injection of photoelectrons from the $\mathrm{MoS}_{2}$. At low illumination powers $\left(\sim 1-6 \mathrm{~mW} \mathrm{~m}^{-2}\right)$ these devices show high responsivities of $5 \times 10^{8} \mathrm{AW}^{-1}$ and $10^{10} \mathrm{AW}^{-1}$ at RT and at $130 \mathrm{~K}$ respectively, with a calculated external quantum efficiency (EQE) of $\sim 32 \%$ at $130 \mathrm{~K} .{ }^{55} \mathrm{~A}$ higher recombination induced by the increase of electron-hole pairs results in decrease of responsivity with increasing illuminating power, to values of $6 \times 10^{7} \mathrm{AW}^{-1}$ at $130 \mathrm{~K}$ for a power of $400 \mathrm{~mW} \mathrm{~m}^{-2}$. The responsivity also decreases for thinner $\mathrm{MoS}_{2}$ flakes, ${ }^{55}$ with similar works showing maximum responsivities for single-layer $\mathrm{MoS}_{2}$ of $\sim 10^{7} \mathrm{AW}^{-1}$ for $10 \mathrm{~mW} \mathrm{~m}^{-260}$ and of $10 \mathrm{AW}^{-1}$ for a power of $2.2 \mathrm{~W} \mathrm{~m}^{-2}{ }^{146}$ The trapped holes in the $\mathrm{MoS}_{2}$ also induce a slower response of the devices, with values over $10^{3}$ s. ${ }^{55}$ As we shall see later, this persistent photocurrent can be used to produce memory devices (see Figure 9b). ${ }^{55}$

Another kind of photodetector relies on the encapsulation of one or several TMD with graphene ${ }^{27,58}$ (Figure 9c). In this case, the TMD is used as the absorption layer, while the graphene layers act as the top and bottom electrodes, owing to its transparency and good electronic properties. If a bias is applied between the two graphene layers or they are doped differently (either chemically or by an electric gating), the photocarriers are extracted from the TMD as a photocurrent. The generated photocurrent is observed only when the areas in which all the layers overlap are illuminated (Figure 9d). ${ }^{27}$ It is worth noting in these vertical devices that the direction of the photocurrent is the same as that of the built-in electric field generated by the gate voltage or by doping the graphene layers. Thus it is possible to modulate the photocurrent with the gate, and hence these devices can be operated at zero bias voltage (Figure 9d). ${ }^{27,58}$ This greatly reduces the dark current that originates from biasing the device, decreasing the power consumption of the devices. The transparency of the graphene electrodes allows for it to cover the active areas of the device. Thus, the collection of the photocarriers can be done vertically instead of the lateral collection occurring with lateral metal contacts. The charge extraction is then faster, which decreases the number of carriers lost due to recombination. This can be seen in the responsivity increase reported for $\mathrm{MoS}_{2} / \mathrm{WSe}_{2}$ stacks with $\left(\sim 10 \mathrm{mAW}^{-1}\right.$ at $\left.920 \mathrm{Wcm}^{-2}\right)$ compared to without 
$\left(\sim 2 \mathrm{mAW}^{-1}\right.$ at $\left.100 \mathrm{Wcm}^{-2}\right)$ graphene electrodes. ${ }^{147}$ This fast extraction, along with the high mobility of graphene, also results in ultrafast photodetectors. Response times of 5.5 ps have been reported on graphene/WSe 2 /graphene photodetectors by Massicotte et al., ${ }^{148}$ which is comparable to that of graphene. These devices present IQE values exceeding $70 \%$ in thin $\mathrm{WSe}_{2}$ devices (3 layers) and low dark currents, although the EQE is low (7.3\%) due to the low optical absorption. Consequently, the EQE can be increased for thicker TMD (up to $50 \mathrm{~nm}$ ), with reported values up to $27 \%\left(\mathrm{MoS}_{2}\right)$, ${ }^{58}$ $33 \%\left(\mathrm{WS}_{2}\right)^{27}$ and $34 \%\left(\mathrm{MoS}_{2} / \mathrm{WSe}_{2}\right.$ junction). ${ }^{147}$ In the case of thin TMDs, responsivities of $2.5 \mathrm{AW}^{-1}$ and $3.5 \mathrm{AW}^{-1}$ have been demonstrated in the case of single- and double-layer $W_{2}$, respectively. ${ }^{149}$ Interestingly, these values were obtained under ambient conditions for a large illumination power $\left(\sim 10^{4}\right.$ $W_{\left(\mathrm{cm}^{-2}\right.}{ }^{-2}$. Introducing a relatively thick $h-\mathrm{BN}$ tunnel barrier between the $\mathrm{MoS}_{2}$ and graphene have recently proved to be an effective method to decrease the dark current in photodetectors (Figure 9e). ${ }^{150}$ Photodetectors with responsivities as high as $180 \mathrm{AW}^{-1}$ were fabricated by using a 7 $\mathrm{nm}$ thick $h-\mathrm{BN}$ layer, with relatively fast response times of $0.25 \mathrm{~s}$.

Configurations in which one of the graphene electrodes is replaced by a metal have also been tested. In these configurations, the TMD makes a Schottky contact with graphene and an almost ohmic contact with the metal (Figure $9 f, g) .{ }^{58}$ An increment of the photocurrent and of the EQE can be observed in this configuration compared with the conventional one, which in the case of $\mathrm{MoS}_{2}$ implies an increase from $27 \%$ to $55 \%$.

Recently, ultrafast photodetection has also been demonstrated on $h-\mathrm{BN} /$ graphene/ $h-\mathrm{BN}$ heterostacks on top of a photonic integrated circuit, and with a top electrolyte gate (Figure 9h). ${ }^{151}$ The large mobility attained by encapsulating the graphene in $h-\mathrm{BN}$ (up to $60000 \mathrm{~cm}^{2} \mathrm{~V}^{-1} \mathrm{~s}^{-1}$ ) resulted in response times as short as $3 \mathrm{ps}$. The use of the integrated waveguide and the top gate allows responsivities up to $0.36 \mathrm{AW}^{-1}$, which is a 3-fold increase with respect to previous graphene photodetectors integrated in a waveguide. ${ }^{151}$

The optical properties of the graphene heterostructures can be also applied for light harvesting, transforming the incident light into an electric current. Heterostructures containing TMDs have become an attractive alternative to traditional semiconductors, owing to their bandgaps in the visible range of the electromagnetic spectrum, their high optical absorption coefficients, and the relative accessibility and abundance of their compounds. The efficiency of ultrathin graphene-based photovoltaic devices cannot directly compete with that of much thicker devices based on conventional semiconductors (e.g. $1 \mu \mathrm{m}$ thick GaAs based solar cells), ${ }^{152}$ but the power densities can be 3 orders of magnitude higher than in conventional solar cells. ${ }^{152}$ Some recent attempts have shown photoelectric conversion efficiency of $3.3 \%$ under solar simulator (AM 1.5) illumination for few-layer graphene/WS heterostructures with an Al bottom electrode. ${ }^{153}$ By inserting a $\mathrm{MoS}_{2}$ film in a graphene/Si Schottky junction solar cell, Tsuboi et al. have shown conversion efficiencies up to $11 \%$ under
AM 1.5 illumination. ${ }^{154}$

\section{c) Light emission}

Graphene heterostructures are also being studied for light emitting diodes (LED) applications. Such LED devices operate by the recombination of electron and holes in the junction of the different layers of the heterostructure. These typically contain a tunnel junction, which in most of the cases is made of $h-\mathrm{BN}$. The main role of graphene in these cases is to inject electrons and holes into the active junction area. Withers et al. demonstrated the possibility to produce highly efficient proofof-concept LEDs composed of mechanically stacked 2D layers (Figure 10a). ${ }^{155}$ The band structure of such LEDs is tailored by inserting a TMD layer between two graphene electrodes. Two additional layers of $h-\mathrm{BN}$ serve as tunnel barriers between the graphene and the TMD, with the latter acting as a quantum well (QW). When a large enough bias is applied between the graphene layers, holes and electrons are tunneled into the QW (Figure 10b). For $h-\mathrm{BN}$ thicknesses of 2 layers or more, the lifetime of the injected carriers is long enough to form excitons that then recombine emitting photons (Figure 10c,d). The wavelength of the emitted photons can be tuned by selecting a specific TMD. Also, several QW can be piled up to increase the efficiency of the LEDs. The EQE achieved at low temperatures (below $150 \mathrm{~K}$ ) is $\sim 1 \%$ for single QW and up to $\sim 8.4 \%$ for devices with four QW, which is comparable to the values of organic LEDs. However, the efficiencies for $\mathrm{MoS}_{2}$ or $\mathrm{MoSe}_{2}$ QWs decrease when increasing the temperature. The efficiency at room temperature can be increased by using $\mathrm{WSe}_{2}$, whose electroluminescence shows an opposed temperature dependence to that of Mo-based TMDs due to the opposite orientation of the spin in the valence and conduction bands on $\mathrm{WSe}_{2}$, which makes the lowest-energy exciton dark (Figure 10e). ${ }^{156}$ This allows the production of LEDs with efficiencies of $\sim 5 \%$ for a single $Q W$ at room temperature and with current densities up to $1000 \mathrm{~A} \mathrm{~cm}^{-2}$, a 250 fold increase compared to $\mathrm{MoS}_{2}$ QWs. ${ }^{156}$ Interestingly, the presence of point defects on the TMD can be exploited to further tune the properties of the 2D LEDs. The defects act as single-photon sources, ${ }^{157}$ emitting spatially localized electroluminescence tuned by adjusting the vertical bias on the heterostructure (Figure 10f). ${ }^{157-159}$ The electroluminescence from the defect-bound excitons present narrow emission lines, located at energies below those of the intrinsic excitons. ${ }^{158}$

\section{d) Increasing the efficiency of optoelectronic devices}

Being extremely thin, the interaction of light with graphene 2D heterostructures is lower than in bulk 3D devices. Methods to increase the amount of absorbed or emitted light by 2D heterostructures without increasing their thickness are of a great importance. The coupling of 2D heterostructures with photonic integrated circuits, such as photonic crystal cavities or waveguides (Figure 9h), can greatly enhance the light adsorption ${ }^{151,160}$ or emission. ${ }^{161}$ In this sense, integration of a LED with a photonic crystal cavity have shown a 4-fold 
enhancement of the electroluminescence, producing a linearly polarized emission along the cavity mode. ${ }^{161}$ The interaction with light has also been improved through the use of plasmonic nanoparticles. An example of this is the observed enhancement in the light absorption induced by $\mathrm{Au}$ nanoparticles $(5-10 \mathrm{~nm})$ deposited on graphene/ $\mathrm{WS}_{2} /$ graphene heterostructures, which result in an increase of the photocurrent by a factor of $10 .^{27}$

\section{e) Memory devices}

One of the strategies to produce memory devices is based on using a floating gate structure. The basic mechanism relies on modulating the conductivity of a channel by adjusting the charge stored in an electrically isolated gate. Bertolazzi et al. realized a nonvolatile cell from a graphene/ $\mathrm{MoS}_{2}$ heterostructure. ${ }^{56}$ The device is composed of a FET with a $\mathrm{MoS}_{2}$ channel and graphene contacts. A floating gate is placed on top of the channel, consisting of few-layer graphene electrically isolated from the rest of the device by a $\mathrm{HfO}_{2}$ dielectric layer (Figure 11a). The polarity of the voltage applied to the floating gate electrode will make that electrons tunnel from the $\mathrm{MoS}_{2}$ channel into the floating gate (positive voltage, program state), or in the reverse direction (negative voltage, erase state). This will induce a shift of the threshold voltage in the transfer characteristics, with ratios of current for the erase/program states of $\sim 10^{4}$ (Figure $11 b, c$ ). As the induced shift depends on the amount of charge stored on the floating gate, multiple levels could be used to store information instead of just two. The charge retention of the memory proved to be enough for a few hours, with the device retaining an erase/program ratio of $\sim 10^{3}$ within $1 \mathrm{~h}$ (Figure 11c). Independently, Choi et al. realized a similar structure but using a $h$-BN barrier. ${ }^{65}$ The $h-\mathrm{BN}$ barrier allowed for similar erase/program ratios that are maintained without significant losses for at least $1400 \mathrm{~s}$. The performance of such memories are comparable or even better than those of organic memories. ${ }^{56,65}$ More recently, a new concept for a twoterminal floating memory without a gate electrode has been proposed. ${ }^{162}$ It consists of a MoS 2 channel, a graphene floating gate and an $h-\mathrm{BN}$ barrier (Figure 11d). By applying drain voltages larger than the operational ones, the graphene can be charged or discharged by tunneling through the $h-\mathrm{BN}$ from the top electrodes. Erase/program ratios as high as $10^{9}$ can be obtained by adjusting the $h$-BN thickness, with retention times over $10^{4} \mathrm{~s}$ (Figure 11e). The absence of the gate in these devices also allows easy production of flexible devices.

By taking advantage of the light sensitivity of some 2D materials, light programmable memories can be also realized. Roy et al. demonstrated the operation of one of those memories, consisting of a graphene FET on top of a multilayer $\mathrm{MoS}_{2}$, assembled by exfoliation and stacking techniques (Figure 9a). ${ }^{55}$ These devices revealed a sensitivity to light, which induces variations in the drain-source currents. These variations do not completely disappear with the light source, and instead present logarithmic relaxation times for periods of $\sim 100 \mathrm{~s}$ (Figure 9b). A similar kind of programmable memories have been studied, consisting of graphene FETs supported on $h-\mathrm{BN} .^{163,164}$ In this case, illumination of the graphene/ $h-\mathrm{BN}$ heterostructure under an applied gate voltage induces an electrostatic doping of the graphene due to defect charge migration on the $h-\mathrm{BN}$ (Figure 11f). ${ }^{163}$

\section{f) Graphene as electrode for FETs and logic gates}

Given its outstanding electronic properties, graphene can act as an ideal contact to other 2D materials. Thus, as we have seen, in many heterostructures graphene plays the role of electrode. An example of this is the use of graphene in highly sensitive $\mathrm{MoS}_{2}$-based $\mathrm{NO}_{2}$ gas sensors. ${ }^{165}$ The ability to tune the work function of graphene with a gate results in the possibility to adjust the Schottky barrier with other materials. ${ }^{166-168}$ This allows the formation of almost ohmic contacts of graphene with TMDs, as opposed to the case of junctions of TMDs with metals. Based on this, graphene can be employed to substitute metal electrodes for conventional devices with other $2 \mathrm{D}$ materials, such as TMD-channel FETs and in more complex logic gates. ${ }^{166,167}$ In these devices, graphene is used as an ohmic contact to the TMD channel (e.g. $\mathrm{MoS}_{2}$ ), and also as an electric contact for the top gate. This allows the fabrication of electronic circuits entirely using $2 \mathrm{D}$ materials. The devices with graphene contacts outperformed those with conventional metal contacts, with a tenfold increase in the measured carrier mobility. ${ }^{166}$ Interestingly, the actual processing techniques for $2 \mathrm{D}$ materials allow for fabrication of all-2D circuits with these geometries at large scales. This have been demonstrated by making, devices including isolated FETs and transistors integrated into logic gates. ${ }^{166}$ Techniques to realize $1 \mathrm{D}$ contacts can also be applied to such heterostructures, which is expected to improve performances. $^{45,169}$

\section{g) Vertical and tunnel FETs}

The lack of a band gap in graphene results in FETs with low on/off ratios, rendering them inadequate for implementation in integrated circuits. To overcome this limitation, graphene can be stacked with other 2D materials to fabricate ultrathin vertical FETs (VFET), in which the current flows in the vertical direction through the different layers. The VFET structure proposed by Britnell et al. is composed of a vertical heterostructure of two graphene electrodes separated by a thin $h-\mathrm{BN}$ insulating barrier (Figure 12a,b). ${ }^{40}$ The device was prepared by the stacking of mechanically exfoliated flakes, which are then encapsulated in $h-B N$. A tunnel current is observed when applying a bias voltage between the two graphene layers. This current can be modulated by shifting the graphene bands with a gate voltage. The weak screening of the gate produced by the bottom graphene layer results in the band structure of both layers being mainly aligned, while the bias can control the difference in the chemical potential. The band structure of graphene is crucial for the VFET to work efficiently, as it allows for large shifts of the bands with the applied gate voltage. Given the small thickness of the VFET, the transit time is expected to be of only a few femtoseconds. 
An on/off ratio of 50 was obtained at RT, higher than those usually achieved for planar graphene FETs at RT $(<10)$. The performance of the VFET can be increased by using a TMD barrier instead of $h-\mathrm{BN}$. Due to the smaller bandgap of the TMD, the changes in the Fermi level of the graphene induced by the back gate are now of the order of the barrier height. The gate can then switch the device between an off state governed by tunneling, and an on state governed by thermionic transport. ${ }^{63}$ This results in a further increase of the on/off ratios, with reported values at RT of $10^{4}$ for an $\mathrm{MoS}_{2}$ barrier (6 layer thickness), ${ }^{40}$ and of $10^{6}$ for $\mathrm{WS}_{2}$ (4-5 layer thickness). ${ }^{63}$ By using graphene as the conducting channel and $\mathrm{MoS}_{2}$ as barrier for the contact with electrodes, devices with on/off ratios up to 100 can be obtained that maintain most of graphene mobility. ${ }^{170}$

By increasing the applied bias voltages, the tunnel current in VFETs with an $h-B N$ barrier have been observed to achieve a relative maximum (Figure 12c). ${ }^{171-174}$ This maximum is followed by a region showing negative differential conductance (NDC), with peak to valley ratios of $\sim 4$ for temperatures $<10 \mathrm{~K}$ (Figure 12c). This behavior is a due to resonant tunneling occurring due to the control of the energy shift of the bands of the graphene layers with the gate and the bias voltages. The performance of the resonant tunnel VFET can be increased by aligning the two graphene layers, resulting in tunneling with energy and momentum conservation and with NDC persisting at RT. ${ }^{50,172}$ One of the important uses of devices with NDC is the fabrication of high-frequency electronic oscillators. A schematic of a high-frequency oscillator made with the resonant VFET coupled to an inductor-capacitor circuit can be seen in the inset of (Figure 12d). ${ }^{172}$ The circuit is able to generate oscillations in the $\mathrm{MHz}$ range (Figure $12 \mathrm{~d}$ ), although the short transition times in the femtosecond range potentially allows to reach the $\mathrm{THz}$ regime. Graphene can also be used as the electrode of TMD-based resonant tunnel diodes. TMD resonant tunnel diodes have been realized by the sequential CVD growth of $\mathrm{WSe}_{2}$ and $\mathrm{MoS}_{2}$ or $\mathrm{MoSe}_{2}$ on epitaxial graphene. ${ }^{105}$ Such TMD resonant tunnel diodes achieved a NDC of 2.2 at RT.

There exists another VFET configuration in which a TMD is vertically contacted by a graphene electrode, while the other contact is a metal electrode (Figure 12e). Such VFETs do not rely on tunnel currents, but on the Schottky contact between the graphene and the TMD. When a metal and a semiconductor are brought into contact, either an ohmic contact or a Schottky barrier is formed. In the latter case, a rectifying behavior can be observed. In the case of graphenesemiconductor junctions, the low density of states of graphene allows the tuning of the height of the Schottky barrier by the application of a gate voltage. ${ }^{166,167,175}$ As already mentioned, the contact can then be tuned from almost ohmic to rectifying, for low and high Schottky barriers respectively. Such kind of 3terminal variable barrier devices, sometimes known as barristors, allow a high modulation of the vertical current. By adjusting the thickness of the TMD, VFETs with on/off ratios at RT of $\sim 1500(\sim 30 \mathrm{~nm} \mathrm{MoS}$ and Ti contact $))^{57} 3 \times 10^{4}(\sim 11$ $\mathrm{nm} \mathrm{WSe} \mathrm{C}_{2}$ and Pt/Au contact), ${ }^{176}$ and of $10^{5}\left(\sim 30 \mathrm{~nm} \mathrm{MoSe} \mathrm{H}_{2}\right.$ and $\mathrm{Au} / \mathrm{Ti}$ contact) have been realized (Figure 12e,f). ${ }^{177}$ As opposed to the tunnel VFETs, here the TMD layer acts as a semiconducting channel instead of as a barrier. Thus, the obtained current densities are in general superior to those of tunnel-based VFETs, with values that that can go up to $3.5 \times$ $10^{5} \mathrm{~A} \mathrm{~cm}^{-2}{ }^{57}$ By replacing the back gate employed in these devices with an ion-gel gate, the voltage operation of the VFETs can be lowered to values below $3 \mathrm{~V}^{178}$ This result is essential for the operation at low power consumption. Integration of several of these VFETs into logic gates can be easily realized. As such, logical inverters with gains up to 2 have been demonstrated by combining a p-type (e.g. WSe ${ }_{2}$ or $\mathrm{Bi}_{2} \mathrm{Sr}_{2} \mathrm{CO}_{2} \mathrm{O}_{8}$ ) and a $\mathrm{n}$-type (e.g. $\mathrm{MoS}_{2}$ ) VFET in series (Figure $12 \mathrm{~g}) \cdot{ }^{57,178}$

\section{h) Transparent and flexible electronics}

Owing to their optical, mechanical and electrical properties, 2D heterostructures of graphene are an interesting option for integration into transparent and flexible electronic devices. Here we will briefly describe some examples of 2D devices mounted on flexible substrates that have been already developed, although more detailed discussions can be found in ref. ${ }^{179}$ for 2D materials in general, and in ref. ${ }^{180}$ for graphene. Different kinds of photodetectors have been demonstrated on flexible substrates, such as PET. ${ }^{27,146}$ Although their performances are not better than on $\mathrm{SiO}_{2}$, this is partially due to a performance increase on $\mathrm{SiO}_{2}$ due to the interference of light. ${ }^{27}$ However, heterostructures on PET can endure bending tests for up to 1000 cycles without any sign of fatigue (Figure $13 a, b) .{ }^{146}$ LEDs based on graphene/ $h-\mathrm{BN} / \mathrm{TMD} / h-$ $\mathrm{BN} /$ graphene heterostructures have been also realized on $\mathrm{PET}$, without any evident lost on the performance under an uniaxial strain of $\sim 1 \% .{ }^{155}$ FETs with $\mathrm{MoS}_{2}$ channel, graphene gate and $h-\mathrm{BN}$ dielectric gate were demonstrated on flexible substrates. ${ }^{66}$ These devices showed mobilities $\left(27 \mathrm{~cm}^{2} \mathrm{~V}^{-1} \mathrm{~s}^{-1}\right)$ only slightly lower than on a rigid $\mathrm{SiO}_{2}$ substrate $\left(45 \mathrm{~cm}^{2} \mathrm{~V}^{-1} \mathrm{~s}^{-1}\right)$. The performance was unaltered under uniaxial strains of up to $1.5 \%$, while for larger strains the performance decrease due damage on the bulk metal electrodes. Flexible floating-gate memories have been demonstrated on PET and PI/PDMS (Figure 13c). ${ }^{162}$ The erase/program ratio on these memories decreased from $10^{9}$ on the rigid substrate to $\sim 10^{4}$, but was able to work for strains up to $19 \%$. As the last example, a transparent tunnel VFET on PET has been realized, performing similar to that on a rigid substrate under strains of $\sim 4 \%$. ${ }^{63}$ Overall, most of the 2D heterostructures presented in the previous sections can be integrated into flexible substrates, which is interesting for emerging fields in wearable electronics. However, further advances are yet required with respect to the fabrication or even the direct synthesis of the flexible heterostructures by low temperature growth techniques.

\section{i) Spintronics and superconductivity}

Graphene is an interesting material for spintronics, with spin diffusion lengths and lifetimes at RT rivaling those of other materials. However, the spin-orbit coupling in graphene is very 
weak, as opposed to the case of certain TMDs. Interestingly, the spin-orbit coupling in graphene can be enhanced by the presence of nearby TMD layers. ${ }^{181-183}$ This enhancement enables the electric-field modulation of spin currents on graphene, while its electronic quality is still preserved. These works are expected to pave the way for future high quality graphene-based spintronic circuits. Graphene has also been employed to realize superconductive states on $\mathrm{NbSe}_{2}$, ${ }^{49,104}$ which is highly unstable in air. Encapsulating by graphene (top) and $h-\mathrm{BN}$ (bottom) protected $\mathrm{NbSe}_{2}$ from ambient degradation, allowing to achieve a superconductive state with $\mathrm{a}_{\mathrm{c}} \sim 2 \mathrm{~K}^{49}$

\subsubsection{Vertical heterostructures with other 2D materials:} Synthesis and applications

Some reports have been published on vertical heterostructures of graphene with other types of metal chalcogenides, mainly GaSe. GaSe is a layered semiconductor with a bandgap of $\sim 2.1 \mathrm{eV}$ for single layer thickness. This gap is direct independent of the number of layers, making it a promising material for optoelectronic applications. GaSe has been grown on graphene by vapor-phase deposition at temperatures of $750{ }^{\circ} \mathrm{C}$ and at low pressures ${ }^{106}$ and by MBE at $400^{\circ} \mathrm{C} .{ }^{107}$ An epitaxial relation has been observed between the GaSe and the graphene, although the relative orientation observed depends on the growth conditions. The GaSe shows misalignments of $\sim 10^{\circ}$ in the vapor-phase deposition growth, ${ }^{106}$ while no misalignment or rotations of $30^{\circ}$ were observed in the case of the MBE synthesis growth. ${ }^{107}$ Photodetectors have been realized by drop casting a solution of GaSe nanosheets on graphene. ${ }^{184}$ Such photodetectors show responsivities between $10^{2}$ and $3 \times 10^{5} \mathrm{AW}^{-1}$ for illumination powers of $10^{2}$ and $10^{-2} \mathrm{mWcm}^{-2}$ respectively (Figure 14a). However, photodetectors prepared by mechanical stacking of exfoliated GaSe thin layers showed lower efficiencies than for $\mathrm{WS}_{2}$ photodetectors. ${ }^{27}$

Non-transition metal chalcogenides have also been synthesized on graphene. The epitaxial growth of single to few-layers of semiconducting $\alpha-\ln _{2} \mathrm{Se}_{3}$ on graphene by physical vapor deposition has been achieved using $\ln _{2} \mathrm{Se}_{3}$ powder as precursor (Figure 14b). ${ }^{109}$ The orientation of $\ln _{2} \mathrm{Se}_{3}$ crystals is also determined by that of the underlying graphene, reflecting the epitaxial growth. The study of $\alpha-\mathrm{In}_{2} \mathrm{Se}_{3}$ heterostructures may be interesting for optoelectronic applications, as it shows a higher photosensitivity than $\mathrm{MoS}_{2} \cdot{ }^{109} \mathrm{Bi}_{2} \mathrm{Se}_{3}$ and $\mathrm{Bi}_{2} \mathrm{Te}_{2} \mathrm{Se}$, both topological insulators, are another example of nontransition metal chalcogenide that have been synthesized on graphene (Figure 14c). ${ }^{108,110}$ The thin layers of $\mathrm{Bi}_{2} \mathrm{Se}_{3}$ show defined orientations on the graphene, indicating the epitaxial growth. ${ }^{108}$ Vertical tunnel devices have been fabricated by mechanically stacking a $\mathrm{Bi}_{2} \mathrm{Se}_{3}$ flake on graphene, with the tunnel transport occurring through the interface at low temperatures. ${ }^{185}$ Spintronics devices have been demonstrated by heterostructures of graphene with other topological insulator, $\mathrm{Bi}_{2} \mathrm{Te}_{2} \mathrm{Se}^{110} \mathrm{~A}$ spin polarized current can be efficiently injected in the graphene, owing to the strong spin- orbit coupling with the $\mathrm{Bi}_{2} \mathrm{Te}_{2} \mathrm{Se}$. In general, thin layers of nontransition metal chalcogenides are currently far less explored than in the case of TMDs, although their exotic properties open new possibilities for their integration in heterostructures. Another interesting approach is the integration of graphene with organic materials or metal-organic hybrids. Covalent organic frameworks (COF) are porous periodic frameworks composed of covalently bonded light-weight elements. ${ }^{16}$ COFs can be semiconducting and photosensitive, and can exhibit relatively large mobilities. Their integration in practical devices is however difficult, as usual synthesis processes produce bulk powder without control on the pore structure. However, 2D COFs have been synthesized on graphene, showing improved crystallinities with respect to their respective bulk phase (Figure 14d). ${ }^{186,187}$ Given their porous nature, the study of 3D heterostructures of graphene and COFs is applicable for applications such as gas absorption and storage. However, 2D heterostructures with COF remain largely unexplored. Graphene has also been used to protect 2D sheets of conjugated polymers from high energy electron beams by encapsulation. ${ }^{23}$ Recently, it has been shown that carbon based nanomembranes can be used to fabricate graphene vertical heterostructures, providing an easy and flexible method for the functionalization of graphene and the engineering of its electronic properties. ${ }^{188}$ Heterostructures with pentacene thin films, another organic material, have also been studied. Pentacene is a flexible p-type semiconductor, with a low cost of fabrication. Therefore, it is widely used on organic thin-film transistors, although it presents a low mobility $\left(\sim 1 \mathrm{~cm}^{2} \mathrm{~V}^{-1} \mathrm{~s}^{-1}\right)$. So far, barristors composed of vertical heterostructures of graphene and pentacene have been reported with on/off ratios of $\sim 10^{4} .{ }^{189,190}$ Integration of such devices into flexible substrates have shown high stability for strains up to $4 \% .{ }^{190}$ Recently, Seo et al. have demonstrated a method to produce $p-n$ junctions on graphene by selective doping with 6,13-bis(triisopropylsilylethynyl) (TIPS) pentacene. ${ }^{191}$ As-deposited TIPS-pentacene does not induce a large doping in graphene FETs. However, the FET characteristics showed n-type doping after laser irradiation, due to the oxidation of the TIPS-pentacene (Figure 14e). The level of doping can be adjusted by the irradiation time or the intensity. By spatially controlling the exposure to the laser, p-n junctions with arbitrary shapes can be patterned on the graphene. ${ }^{191}$ Self-assembly is also used to produce thin films of organic molecules on graphene, such as perylene-3,4,9,10tetracarboxylic dianhydride (PTCDA), ${ }^{192}$ oleylamine $^{193}$ or phthalocyanine (Figure 14f). ${ }^{194}$ Such molecules present ordered patterns and relatively strong interactions with the graphene, ${ }^{194}$ and can be employed to modify its electronic properties. ${ }^{193}$ By adjusting the temperature of the graphene during the epitaxial deposition of certain molecules, it is possible to obtain ultrathin organic heterostructures with a precise control on the number of layers and the crystallinity of the deposited molecules. ${ }^{195}$ By the successive growth of layers of different organic molecules, ultrathin vertical $p-n$ junctions can be easily produced, showing a good rectifying behavior and with a photoresponsivity of $\sim 0.37 \mathrm{mAW}^{-1} .195$ 
Perovskite materials are those that share the structure of $\mathrm{CaTiO}_{3}$. Due to their remarkable optical properties, layered inorganic-organic perovskites have attracted a recent interest for their application in photovoltaic applications. ${ }^{112}$ Thus, studies on their integration with other 2D materials, especially graphene, have been rising for the last few years. ${ }^{112,196}$ Niu et al. have demonstrated the direct growth of a perovskite (methylammonium lead halide)/graphene heterostructures (Figure 14g). ${ }^{112}$ Their method consists of the PVD growth of $\mathrm{Pbl}_{2}$ nanoplatelets on the graphene, followed by the conversion into perovskite by the reaction with $\mathrm{CH}_{3} \mathrm{NH}_{3} \mathrm{I}$. Cheng et al. have devised a similar method in which $\mathrm{Pbl}_{2}$ crystals are mechanically exfoliated and deposited on the graphene. ${ }^{196}$ Encapsulation with 2D materials is also essential to protect the perovskite, which is unstable in ambient conditions. ${ }^{196}$ Patterning the 2D substrate before the growth of the perovskite allows to obtain patterned heterostructures. ${ }^{112}$ Vertical heterostructures of perovskite encapsulated by two graphene electrodes show photocurrents on the overlapping areas, with responsivities of $\sim 950 \mathrm{AW}^{-1}$ and response times of a few tens of ms (Figure 14h). ${ }^{196}$

\subsection{In-plane heterostructures with 2D materials}

While vertical heterostructures of $2 \mathrm{D}$ materials can be prepared by physically stacking the different layers, the fabrication of in-plane heterostructures with truly 1D contacts require more advanced synthesis methods. Advances on the synthesis procedures have allowed the development of such in-plane heterostructures of graphene with other 2D materials. The combination of metal, semiconductor and insulator 2D materials as in-plane heterostructures, is interesting for the development of continuous, single-atom thick, in-plane integrated circuits with ultimately thin contacts. ${ }^{31}$

Probably one of the first examples of synthesis of such in-plane heterostructure is that of $h-\mathrm{BN}$ and graphene in-plane hybrid sheets simultaneously grown during a single CVD process with $\mathrm{CH}_{4}$ and ammonia borane as precursors. ${ }^{197}$ The resulting sheets consisted of randomly located patches of both $h-\mathrm{BN}$ and graphene, and with thicknesses from a single to a few layers. This method produces hybrid sheets with a fixed ratio of the graphene and $h-\mathrm{BN}$, but does not provides control in the size or shape of the domains and their interfaces. To obtain controlled in-plane heterostructures, two-step growing processes were developed in which the materials are grown at different stages. In-plane heterostructures of graphene and $h-$ BN with controlled shape and sharp interfaces can thus be produced by patterning a CVD-grown graphene layer and then selectively growing the $h-\mathrm{BN}$ by a second $\mathrm{CVD},{ }^{30}$ or in the opposite order (Figure 15a). ${ }^{32,33}$ The direct growth of elaborate 2D graphene-based devices, such as multi-terminal transistors or band pass filters, are possible with this approach. Even though there is a slight mismatch on the crystal lattice of graphene and $h-\mathrm{BN}$ ( $1.7 \%)$, STM and HR-TEM studies indicated that it is possible to achieve a seamless stitching at atomic scale between both (Figure 15b). ${ }^{33-35}$ The two lattices are then oriented, with the junctions corresponding predominantly to zigzag edges. TEM inspections also showed evidences of hybridization, with bonding occurring between the $\mathrm{N}$ atoms of the $h-\mathrm{BN}$ and the $\mathrm{C}$ atoms of the graphene. ${ }^{35}$ These findings agree with DFT calculations, which show that zigzag junctions are more energetically favorable than armchair junctions, while there is not a large energy difference between $\mathrm{C}-\mathrm{N}$ and $\mathrm{C}-\mathrm{B}$ bonds and hence the kind of hybridization responds to the edge termination of the $h-\mathrm{BN}$ layer. ${ }^{3,198,199}$ In-plane hybrid sheets can also be obtained on dielectric $\mathrm{SiC}(0001)$ surfaces by a two-step growth processes. ${ }^{34}$ A $h$-BN layer is firstly grown by CVD on the SiC, while a subsequent annealing step at high temperatures introduces some graphene regions. The orientation of the hybrid sheet obtained in this way is completely determined by that of the underlying SiC. In-plane graphene/ $h-\mathrm{BN}$ junctions have been recently reported to grow in a TEM chamber (Figure 15b). ${ }^{35}$ Graphene grows from the edge of $h-\mathrm{BN}$ layers supported on graphene by the supply of carbon atoms from residual hydrocarbons in the chamber. Graphene islands can also be grown on holes performed on the $h-\mathrm{BN}$ by the electron beam, opening the possibility to produce quantum confined heterostructures with a high control.

In-plane heterostructures of graphene with some TMDs have been recently realized, although the works are yet scarce compared to the case of $h-\mathrm{BN} .^{200-203}$ In all of them, patterned heterostructures are obtained by pre-patterning the first material (either the graphene or the TMD) and then growing the second material by CVD (Figure 15c). A perfect planar stitching of the layers have not been realized so far, with TEM measurements showing an overlapping in the junction with sizes going from a few $\mathrm{nm}$ to some tens of $\mathrm{nm} \cdot{ }^{200,201}$ Evidence of weak van der Waals interaction in the overlapped region instead of covalent bonding between the two materials has been reported, owing to the large lattice mismatch between the two lattices. ${ }^{200}$ Consequently, no relation between the orientation of the two lattices have been reported, indicating that the edges simply act as nucleation centers but the growth is not epitaxial (Figure 15d,e). ${ }^{200,201}$ However, good ohmic contacts between the graphene and the TMD have been reported, making this a perfect method to integrate graphene as electrode for 2D devices. ${ }^{201,202}$ This, along with the scalability of the employed methods for the CVD synthesis, allows for the fabrication of high quality devices at large scales, including FETs, diodes, and NAND, NOR and inverter gates (Figure 15f,g). ${ }^{200,201}$

\subsection{Heterostructures with lower dimensionality materials}

In this section we will discuss heterostructures of graphene with lower dimensionality materials. Carbon nanotubes (CNTs) are one-dimensional structures composed of carbon atoms, and with a structure similar to that of graphene. Graphene heterostructures with CNTs have been prepared, either by deposition of aligned CNT arrays on the graphene, ${ }^{204}$ or by the CVD synthesis of graphene on a $\mathrm{Cu}$ substrate covered by functionalized CNTs (Figure 16a). ${ }^{205}$ In these structures, the CNTs are connected to the graphene sheet either by covalent 
or $\pi-\pi$ bonds. The presence of the CNTs provides a reinforcement to the graphene, and hence the name "rebar" graphene given sometimes to the heterostructure, from "reinforcement bar". The rebar graphene is strong enough to endure free-standing handling, with the possibility to realize polymer free transfer from the $\mathrm{Cu}$, or to use it as a holder for TEM specimens (Inset of Figure 16a). A further reinforcement can be attained by performing a second CVD with Fe particles deposited on the rebar structure. ${ }^{206}$ Rebar graphene with $h-$ BN nanotubes have been also demonstrated, showing mechanical properties similar to those of the CNT rebar. ${ }^{207}$ Other than for reinforcement, heterostructures of graphene with CNTs have been applied to electronic and optoelectronic devices. Broadband photodetectors composed of graphene and single-wall CNTs (SWNT) films showed responsivities of $120 \mathrm{AW}^{-1}$, with expected values $\sim 1000 \mathrm{AW}^{-1}$ for lower illumination powers (Figure 16b). ${ }^{208}$ The fabrication consists of coating a $\mathrm{SiO}_{2} / \mathrm{Si}$ substrate with a thin SWNTs network film and then transfer the graphene and fabricate the electrodes. As graphene retains its mobility almost intact, the response time of these photodetectors can be as low as $\sim 100 \mu$ s. Owing to its flexibility and transparency, graphene heterostructures with SWCNTs have also been used as electrode in CNT-based flexible electronics. ${ }^{209}$ The heterostructure was used for making the drain, source and gate electrodes of FET devices, for which the channel is a SWNT network. The whole device showed on/off ratios up to $10^{5}$ and mobilities of $40 \mathrm{~cm}^{2} \mathrm{~V}^{-1} \mathrm{~s}^{-1}$, which were stable for strains up to $20 \%$.

Heterostructures of graphene with zero-dimensionality materials have also been realized, posing a special interest for the increase of sensitivity of photoactive graphene devices. The responsivity of a purely graphene photodetector is usually $\sim 10 \mathrm{mAW}^{-1}$. By depositing a thin film $(\sim 80 \mathrm{~nm})$ of $\mathrm{PbS}$ quantum dots (QD) on bilayer graphene, photoresponsivities up to $10^{7}$ $\mathrm{AW}^{-1}$ have been reported by Konstantatos et al. (Figure 16c). ${ }^{210}$ This ranks among the highest sensitivities reported for a graphene based photodetectors. The improvement in the responsivity is due to generation of photocarriers in the $\mathrm{PbS}$ QDs and transferred to the graphene, along with the high carrier mobility of the graphene channel. The response times are however comparatively long, in the scale of seconds. The attained EQE is also of only $25 \%$, although it can be increased to values of $80 \%$ by using a top gate to direct the generated photocarriers to the graphene channel in a more efficient way. ${ }^{211}$ These devices can also be realized on flexible PET substrates and still maintain their efficiency after bending tests of 1000 cycles (Figure 16d). ${ }^{212}$ Apart from these, there exist theoretical and practical studies about the possibility to assemble more exotic heterostructures. ${ }^{213,214}$

\subsection{Intercalation}

Intercalation of foreign species in the space between layers is a procedure employed for a wide range of layered materials and intercalants. A recent comprehensive review on the intercalation on 2D materials can be found in ref. ${ }^{215}$. Here, we will introduce some of the most pertinent results on the intercalation of double- and few-layer graphene supported on a substrate. Intercalation of atomic or molecular species on the interlayer spaces of graphite, named graphite-intercalation compounds (GIC), is widely used to modify its physical and electrical properties. The space existing between the layers can also be used as a nanoreactor to produce reactions that require extreme pressures. ${ }^{216}$ The van der Waals interaction between the layers provides in localized areas of high pressures, which are able to induce chemical reactions between intercalated molecules. Intercalation of FLG has also led to the realization of magnetic, ${ }^{217,218}$ and superconducting ${ }^{219,220}$ 2D states for $\mathrm{FeCl}_{3}$ and $\mathrm{Ca}$ as intercalants, respectively.

Intercalation of $\mathrm{FeCl}_{3}$ in BLG and $\mathrm{FLG}$ have been widely investigated as a way to decrease the sheet resistance while keeping a high optical transmittance (Figure 16e-g). It is generally conducted by chemical vapor transport, with the graphene and the $\mathrm{FeCl}_{3}$ kept in separated zones of a glass ampoule sealed in vacuum. ${ }^{217,221} \mathrm{~A}$ differential of temperatures is then applied to the ampoule, with the graphene area being in the hot zone $\left(\sim 340-350^{\circ} \mathrm{C}\right)$, while the $\mathrm{FeCl}_{3}$ is maintained at a lower temperature $\left(\sim 290-320^{\circ} \mathrm{C}\right)$. The intercalation usually requires a few hours to complete $(\sim 10 \mathrm{~h})$, after which the ampoule can be brought to room temperature. By using this setup, Zhan et al. demonstrated the $\mathrm{FeCl}_{3}$ intercalation in exfoliated graphene flakes with thicknesses of 2, 3 and 4 layers. ${ }^{221}$ The intercalation can be homogeneous and show a high stability in air, with no evidence of degradation occurring for as long as 1 year in the best reported cases. ${ }^{221-223}$ Intercalation is stable in air even under high humidity conditions for a few weeks (relative humidity up to $100 \%$ ), or at temperatures of $150{ }^{\circ} \mathrm{C}$ for periods of hours. ${ }^{224}$ The charge transfer of the graphene can be monitored by Raman spectroscopy, which shows a stiffening of the $G$ and $2 D$ bands indicating a p-type doping of the graphene layers (Figure 16f). ${ }^{221,222}$ Calculations from Raman results provide an estimated shift of the Fermi level of $\sim 0.9 \mathrm{eV}$, corresponding to a hole density of $\sim 5.8 \times 10^{14} \mathrm{~cm}^{-2}$ for the stage- 1 intercalated samples. ${ }^{222}$ These values are on the same order of magnitude to those obtained from conductivity measurements $\left(\sim 5.8 \times 10^{14} \mathrm{~cm}^{-2}\right){ }^{223}$ After the intercalation, the $2 D$ band of few-layer graphene can be fitted by a single Lorenztian, ${ }^{222}$ indicating a decoupling between the layers induced by the increase of the inter-layer distance. This decoupling has been corroborated by observing the Shubnikov-de Haas oscillations of the longitudinal resistance in a perpendicular magnetic field of intercalated $B L G,{ }^{217}$ while partial decoupling has been observed in the case of FLG due to unintentional deintercalation in the two upper layers of the stack during the processing. ${ }^{223}$ The different environments to which the layers are exposed for thicknesses of 3 and more layers is evidenced by the splitting of the $G$ band into two modes (Figure 16f). ${ }^{21,222}$ The mode at lower wavelengths $\left(G_{1}\right)$ arises from the surface layers (top and bottom), while the mode at higher wavelengths $\left(G_{2}\right)$ corresponds to inner layers surrounded by $\mathrm{FeCl}_{3}$ on both sides (Figure 16e). ${ }^{221}$ By decreasing the intercalation time, selective intercalation of 
$\mathrm{FeCl}_{3}$ between some of the layers in FLG has been recently reported, ${ }^{225}$ which could result in the opening of a bandgap owing to the vertical electric field caused by the asymmetrical doping.

In terms of electronic properties, the hole doping caused by the $\mathrm{FeCl}_{3}$ intercalation on few-layer graphene leads to a decrease of the sheet resistance. Pristine, single-layer graphene has a high optical transmittance ( $97.7 \%)$, but its sheet resistance is high (usually over $1000 \Omega$ /sq for undoped single layer graphene) for the use of graphene as transparent electrode. Sheet resistance in FLG is still of a few hundred of $\Omega /$ sq. After intercalation with $\mathrm{FeCl}_{3}, \mathrm{FLG}$ shows a decrease of the sheet resistance to values as low as $8.8 \Omega / \mathrm{sq}$ at room temperature for a thickness of 5 layers. ${ }^{223}$ Interestingly, the intercalation does not severely decrease the optical transmittance of the FLG, that retains a value of $84 \%$ at 550 $\mathrm{nm}$ compared with the $88.5 \%$ for pristine 5 layers. These values can compete with those of ITO, with transparencies of $90 \%$ and sheet resistances between 10 to $50 \Omega$ /sq (Figure $16 \mathrm{~g}$ ). The relatively low temperatures employed for the intercalation and the inherent mechanical properties of FLG, allow for their use as flexible and transparent substrates, which provides a clear advantage compared with the rigid ITO. Overall, $\mathrm{FeCl}_{3}$ intercalated FLG is an interesting alternative material to the commonly employed ITO for diverse optoelectronic applications.

Another materials have been intercalated into substrate supported FLG, one of the most promising being Li. In-situ measurements on electrochemical intercalation of Li on FLG have recently been demonstrated to $n$-dope the FLG and decrease its sheet resistance (Figure 16h). ${ }^{226}$ Remarkably, the intercalation of $\mathrm{Li}$ also increases the optical transmittance of the FLG. On relatively thick flakes of 19 layers, optical transmittances up to $91.7 \%$ have been attained, with sheet resistances as low as $3 \Omega /$ sq (Figure 16i). The small size of a $\mathrm{Li}$ atom make it also convenient as an intermediate for the intercalation of larger atoms, such as $\mathrm{Ca}^{219,220} \mathrm{~A}$ detailed revision of graphene heterostructures for Li-ion batteries will be carried on in the section 4 .

\section{Solution-processed heterostructures}

\subsection{Scalable exfoliation of 2D materials}

Layered 2D materials can be produced by a variety of techniques as discussed in the previous sections, and while the material created by both micromechanical exfoliation and CVD are of high quality and large crystal size, these production methods lack scalability and remain costly, preventing their use for many applications. Wet chemical processes, such as liquid phase ultrasonication and electrochemical intercalation, can currently produce large volumes of dispersed monolayer and few layer flakes of various 2D materials, cheaply and with the potential for scale-up. ${ }^{14,227-233}$ These liquid-based exfoliation techniques allow the easy creation of thin films, coatings, or additives consisting of a large number of nanosheets, which is ideal in applications such as energy generation and storage. These different scalable liquid phase exfoliation techniques can be visualised schematically in (Figure 17).

The first large scale method of liquid exfoliation of 2D materials was for graphene oxide (GO), where graphite is first treated with strong oxidising agents such as sulphuric acid and potassium permanganate, which increases the hydrophilicity of the graphite. This increased hydrophilicity allows the graphite layers to be easily intercalated by water and separated by mild ultrasonic treatment, producing flakes down to monolayer thickness and hundreds of nanometres in lateral dimension in concentrations up to $1 \mathrm{mg} / \mathrm{ml}^{234}$ Intercalation of ionic species, typically by electrochemical methods, into a variety of layered species beyond simply graphite leads to increased interlayer spacing and the subsequently weakened interlayer coupling allows the material to be easily exfoliated. ${ }^{229}$ The resulting dispersion can also be stabilized by surfactants. Intercalation can also produce large quantities of monolayer flakes with dimensions of a few hundred nanometres. Ultrasonic treatment in specially chosen solvents can lead to direct exfoliation of many 2D materials. ${ }^{235-237}$ The ultrasonic sound waves produce cavitation bubbles, causing the $2 \mathrm{D}$ material to break up into individual nanosheets. Each of these techniques is able to produce large volumes of exfoliated nanosheets dispersions which can subsequently be processed to form a variety of devices. Although the quality of such exfoliated material is typically lower than that produced using mechanical cleavage or CVD, it is sufficient for a wide range of applications, particularly when different 2D materials are combined to form hybrid nanocomposites.

\subsection{Formation of graphene-based hybrid composites}

There has been an enormous effort focused on exploiting the many beneficial properties of 2D materials including graphene, however typically what is desired for the best device performance is a variety of properties from different materials. This has led to the exciting area of hybrid composite research; where multiple 2D materials are mixed together to synergistically improve the performance over any single component. This typically involves the addition of highly conductive graphene-derivatives to mixtures of other layered materials to increase the conductivity, while simultaneously altering the morphology and surface area of the resultant hybrid. The most common example of these hybrid composites, due to its applicability for electrical devices, is the TMD/graphene hybrid composite. Such composites of TMDs and graphene allow the conductivity of graphene to be exploited, while maintaining the unique chemistry of the TMD. ${ }^{238-240}$ Of these TMD/graphene composites studied so far the most common, due to its relative abundance and ease of production, is the $\mathrm{MoS}_{2}$ /graphene hybrid composite, which can act as a proxy for the performance of many similar TMDs. However, different TMDs, as well as other layered materials, have also gained interest for use in graphene based composites and these will be discussed in further detail later. 
These hybrid composites can be produced by several methods. The simplest is to exfoliate each of the individual 2D materials separately and then mix the dispersions together. This can be achieved by producing exfoliated dispersions of a selected material and then generating a film either by filtration onto a membrane or drop-casting directly onto the desired substrate. An example of this mixing procedure to form a $\mathrm{MoS}_{2}$ /graphene composite is shown schematically in (Figure 18a) and an SEM image showing the resultant layered composite structure is shown in (Figure 18b). ${ }^{241}$ This procedure has the benefit of being very simple and easily scalable. However, the interaction between each material typically occurs only when the solvent is removed from the dispersion, causing the mixture of flakes to form a composite film. By varying the relative ratio of each component it is possible to tune the properties of the laminar structure that is produced using this technique, to optimise performance for the desired application. ${ }^{242-245}$

Hydrothermal synthesis is another process that is widely used to produce these hybrid nanocomposites. The hydrothermal growth process typically involves the addition of an aqueous dispersion of chemical precursors in a sealed vessel, which is then heated $\left(\sim 200{ }^{\circ} \mathrm{C}\right)$ in an autoclave to form the desired crystalline material. ${ }^{246-249}$ Solvothermal growth is similar except that instead of water, a non-aqueous solvent is used. This in situ growth process allows for a close interaction between the different materials, with crystals of $\mathrm{MoS}_{2}$ being able to be grown directly on the surface of a graphene supporting layer to form the hybrid composites. Figure $18 \mathrm{c}$ shows schematically the process to hydrothermally produce a $\mathrm{GO} / \mathrm{MoS}_{2}$ hybrid composite structure. ${ }^{250}$ This was achieved by first taking Li-intercalation exfoliated $\mathrm{MoS}_{2}$ and mixing by ultrasonication with a GO dispersion, before treating at $180^{\circ} \mathrm{C}$ for $24 \mathrm{~h}$. This was then chemically reduced by $\mathrm{N}_{2} \mathrm{H}_{4} \cdot \mathrm{H}_{2} \mathrm{O}$ at 90 ${ }^{\circ} \mathrm{C}$ for $12 \mathrm{~h}$ followed by freeze-drying to produce a porous structure. Due to the high temperatures that the dispersions are exposed to, the interaction between the $\mathrm{MoS}_{2}$ and GO is strengthened, leading to good structural stability and fast charge transport. This freeze-drying process also allows for the formation of a highly porous 3D aerogel structure, which maximises available surface area. The formation of such a porous structure avoids the common drawback of solution exfoliated processes, which is the restacking of the exfoliated material which reduces available surface area and overall performance of subsequent devices. Figure 18d shows an SEM image illustrating this high porosity of the resulting $\mathrm{MoS}_{2}$ /graphene composite structure, where the ratio of $\mathrm{MoS}_{2}$ :graphene is $80: 20$. This simple and scalable procedure allows the formation of a unique 3D structure where the relatively larger graphene sheets are decorated with smaller $\mathrm{MoS}_{2}$ nanoflakes. This nanostructured morphology, with its high surface area conductive network of exfoliated material, is ideal for many applications, but in particular for electrochemical energy storage. ${ }^{251}$

\subsection{Hybrid composite supercapacitors}

2D materials are excellent candidates for electrochemical energy storage due to their high available specific surface area and versatile electronic structure. Electrochemical energy storage, such as lithium ion batteries or supercapacitors, are widely used in portable electronic devices, electric transportation, and even grid scale systems. The application of graphene in energy storage technology has been investigated widely due to the many excellent properties such as mechanical strength, high specific surface area $\left(2675 \mathrm{~m}^{2} / \mathrm{g}\right)$ and high electrical conductivity. ${ }^{252-254}$ Similarly-structured layered TMDs, particularly $\mathrm{MoS}_{2}$, have so far attracted less interest but are gaining increased popularity for integration into energy generation and storage devices due to similarities with graphene. ${ }^{255,256}$ Despite the increasing interest in the electrochemical properties of $\mathrm{MoS}_{2}$ the fundamental charge storage processes are still not well established, with some disagreement between reports of its electron transfer characteristics and pseudocapacitance. ${ }^{257-259}$ Hybrid composites consisting of multiple $2 \mathrm{D}$ materials are gaining increased interest for applications in energy storage and generation. This is due to the beneficial synergy that occurs when the high conductivity of graphene, can be used to enhance the intrinsic charge storage properties of another material, such as $\mathrm{MoS}_{2}$. Examples of such hybrid composites include graphene/metal oxides ${ }^{243,260-262}$ as well as graphene/TMD composites which will be discussed in further detail here. ${ }^{242,244,245,250,263-273}$

Electrochemical supercapacitors, sometimes referred to as ultracapacitors, have attracted increasing attention for their rapid charging capabilities, very long cycle lifetime and wide operating temperature range. ${ }^{274}$ The charge storage mechanism in supercapacitors consists of a combination of electric-double layer capacitance (EDLC), where electrolyte ions accumulate at the electrode interface, as well as pseudocapacative ion absorbance or intercalation where rapid redox processes can occur. As no chemical reactions are typically occurring during the charge/discharge of the device, supercapacitors can operate on very short time scales (i.e. high power density) with very long cycle lifetimes. Supercapacitor devices consist of two electrodes (either symmetrical or asymmetrical) separated from each other and filled with an electrolyte solution. An ideal electrode material is one that has both high conductivity and surface area to maximise the amount of charge stored in the double layer at the interface. This makes exfoliated graphene an ideal electrode material, with several reviews previously discussing the benefits of graphene for supercapacitor applications. ${ }^{254,275,276}$

The formation of hybrid composites of graphene with other 2D materials allows the optimisation of their performance by tuning the conductivity and morphology of the composite structure. Figure 19 shows an example of a graphene/MoS composite supercapacitor device created using solution based exfoliation processes. ${ }^{245}$ Commercially available graphene powder was first ultrasonicated to produce a stable dispersion, and a dispersion of $\mathrm{MoS}_{2}$ was similarly produced by solution exfoliation (seen in Figure 19a). These dispersions were then mixed and filtered to create thin $(\sim 500 \mathrm{~nm})$ flexible films as 
electrodes in a symmetrical supercapacitor arrangement using an aqueous electrolyte. Figure $19 \mathrm{~b}-\mathrm{d}$ shows a schematic of the coin-cell architecture that was used to test the composite films, and demonstrates the flexibility of the thin film electrodes. The use of this coin-cell structure allows for a realworld investigation of the performance for possible commercial applications. ${ }^{277}$ When tested using an aqueous electrolyte $\left(\mathrm{Na}_{2} \mathrm{SO}_{4}\right)$ it was found that the graphene by itself exhibits moderate performance and behaved as an ideal double-layer capacitor, as established in literature. ${ }^{254}$ On the other hand, $\mathrm{MoS}_{2}$ suffered from high internal resistance due to its semiconducting nature and so produced a lower capacitance. However, as seen in Figure 19e, when the graphene and $\mathrm{MoS}_{2}$ dispersions were combined in a 1:3 ratio the performance was significantly enhanced over either individual component. This was attributed to a combination of physical interaction between the two different materials, as the smaller $\mathrm{MoS}_{2}$ flakes could coat and prevent restacking of the larger graphene sheets, as well as the increased conductivity that occurs due to the network of graphene flakes and the ion adsorption pseudocapacitance that occurs due to the $\mathrm{MoS}_{2} \cdot{ }^{245}$ It has also been observed that with repeated charge/discharge cycling the measured capacitance can increase over time, due to the repeated intercalation/deintercalation of the electrolyte ions $\left(\mathrm{Na}^{+}\right)$into the composite film, leading to increased active surface areas with time. ${ }^{245,278,279}$

Maximising the electrode surface area and preventing restacking by creating a porous $3 \mathrm{D}$ structure is another route to increase device performance. Figure 19f,g shows the morphology of a $\mathrm{MoS}_{2}$ /graphene aerogel structure produced by hydrothermal synthesis, illustrating the high available surface area. Aerogel structures are ideal for energy storage applications as they provide increased active surface area by forming a large interconnected network of 2D materials. ${ }^{269,280,281}$ This aerogel structure allows the high specific surface areas of many 2D materials to be more properly exploited, but also suffers from issues with long term stability. ${ }^{282}$ The relative charge/discharge behaviour of this $\mathrm{MoS}_{2}$ /graphene aerogel electrode when tested in a supercapacitor device (Figure 19h), showed that the composite electrode outperformed either of the single component devices. This 3D structured electrode demonstrated both high capacity and stability with extended cycling due to the flexible nature of the graphene, showing that 3D hybrid composite structures are an ideal electrode morphology for electrochemical energy storage. ${ }^{270}$

Naturally occurring $\mathrm{MoS}_{2}$ exists in the stable $2 \mathrm{H}$ phase (Figure 2c), which possess a trigonal prismatic lattice arrangement and is semiconducting, as discussed previously. This can be distorted, typically through charge injection by intercalation from lithium ions, to form the metallic $1 T$ phase which has an octahedral arrangement. ${ }^{283,284}$ For energy storage applications, where conductivity of the electrode material is vitally important, it is beneficial to increase the percentage of the 1T polymorph present in the electrode material. The synthesis of a hybrid composite aerogel structure consisting of a mixture of
$\mathrm{MoS}_{2}$ in both the metallic $1 \mathrm{~T}$ and semiconducting $2 \mathrm{H}$ phase, combined with reduced graphene oxide $(\mathrm{rGO})$ is shown in (Figure 20). ${ }^{268}$ This mixture of crystal lattice structures is important as the metallic $1 \mathrm{~T}$ phase has already been shown to provide improved electrochemical charge storage properties due to a combination of its conductivity and the ability of the exfoliated and restacked $\mathrm{MoS}_{2}$ sheets to intercalate various electrolyte ions. ${ }^{285}$ The $1 \mathrm{~T} / 2 \mathrm{H}-\mathrm{MoS}_{2} /$ graphene composite structure was produced by forming $\mathrm{MoS}_{2}$ in situ through the reduction of phosphomolybdic acid hydrate $\left(\mathrm{H}_{3} \mathrm{PMo}_{12} \mathrm{O}_{40} \cdot \mathrm{xH}_{2} \mathrm{O}\right)$ in the presence of a sulphur precursor ( $\mathrm{L}$ cysteine). These precursors were combined with a GO dispersion and underwent hydrothermal treatment $\left(180^{\circ} \mathrm{C}\right.$ for $12 \mathrm{~h}$ ), followed by freeze drying to make the aerogel shown in (Figure 20a). In this work the in situ growth of the $\mathrm{MoS}_{2}$ directly onto the $\mathrm{GO}$ produces a strong interaction between the two materials, allowing for not only a robust 3D structure to be formed but also beneficial electrochemical performance. By maximising the interaction between the different materials in the heterostructure composite the charge transfer between the two is also maximised, leading to beneficial performance. These 3D networks of 2D materials are ideal for supercapacitor applications and (Figure 20b) compares the relative energy storage performance of the $\mathrm{rGO}$ aerogel by itself to the composite structure, clearly the composite performance is far superior to the single component. Hybrid composites made by simply mixing commercial $\mathrm{MoS}_{2}$ powders with $\mathrm{rGO}$ were also produced ( $r G O-\mathrm{MoS}_{2}-\mathrm{p}$, green curve) and compared to the in situ co-synthesised composite ( $\mathrm{rGO}-\mathrm{MoS}_{2}-\mathrm{co}$, blue), as well as $\mathrm{MoO}_{2}$ /graphene composites co-synthesised without the sulphur pre-cursor ( $\mathrm{rGO}-\mathrm{MoO}_{2}$, red). From the results shown in (Figure 20b) the superior performance of the in situ grown $\mathrm{MoS}_{2}$ can clearly be seen, illustrating the increased capacitance that can be achieved due to the close interaction between the two different materials. The high stability of this in situ produced composite structure was also demonstrated by measuring the change in capacitance with repeated cycling with very little degradation in performance over 50,000 cycles, better than either the $\mathrm{rGO}$ or $\mathrm{MoS}_{2}$ single component electrodes which reduced dramatically after 13,000.

The interaction between different $2 \mathrm{D}$ materials present in a hybrid composite can have an enormous effect on the electrochemical energy storage performance and requires further investigation. It has been shown that the interaction between $\mathrm{rGO}$ and $\mathrm{MoS}_{2}$ in a hybrid composite can even lead to a phase transition of the $\mathrm{MoS}_{2}$ lattice, shown schematically in Figure $20 \mathrm{c}$, from the $2 \mathrm{H}$ to the $1 \mathrm{~T}$ phase. ${ }^{286}$ Mahmood et al. demonstrated that when $\mathrm{MoS}_{2}$, produced by ultrasonication of bulk $\mathrm{MoS}_{2}$ powder, was mixed with a dispersion of $\mathrm{GO}$ and then heated to $200{ }^{\circ} \mathrm{C}$ for $12 \mathrm{~h}$ under vacuum before filtering to form a composite structure a unique interaction occurred. This procedure also thermally reduces the $\mathrm{GO}$ layers forming rGO. The hybridisation between the $\mathrm{MoS}_{2}$ and rGO nanosheets during the high temperature treatment forms a unique interlayer coupling, where negative charge was transferred from the $\mathrm{GO}$ into the $\mathrm{MoS}_{2}$ lattice leading to the observed phase transition. Evidence of this phase transition can be seen 
in the TEM image shown in Figure 20d. The $\mathrm{MoS}_{2}$ lattice in direct contact with the $\mathrm{rGO}$ at the interface shows an octahedral orientation, indicative of the $1 T$ phase, while layers that are not in contact show the typical $2 \mathrm{H}$ trigonal prismatic lattice structure. The transfer of negative charge from the GO into the closest $\mathrm{MoS}_{2}$ layer destabilises the $2 \mathrm{H}$ crystal lattice and favours the octahedral coordination. ${ }^{287}$ Typically the $1 \mathrm{~T}$ phase is metastable, reverting to the lower energy $2 \mathrm{H}$ phase over time. However, DFT calculations indicated that the interaction between the $1 \mathrm{~T}-\mathrm{MoS}_{2}$ and graphene are enhanced, providing a higher energy barrier for the $1 \mathrm{~T}$-to- $2 \mathrm{H}$ transition allowing the unstable $1 \mathrm{~T}$ phase to exist at the interface. ${ }^{286}$ As discussed previously the presence of this $1 \mathrm{~T}-\mathrm{MoS}_{2}$ phase can greatly improve the energy storage properties of a supercapacitor device, and so the presence of this mixed phase is beneficial for energy storage applications. ${ }^{285}$ This increased performance of the $1 \mathrm{~T}$ phase is related to the charge storage mechanisms present, which are a combination of proton intercalation in between layers of $\mathrm{MoS}_{2}$ and adsorption of the protons onto the surface of the layers. Figure 20e demonstrates the enhanced electrochemical charge storage behaviour that occurs from producing a $\mathrm{rGO} / \mathrm{MoS}_{2}$ composite (2D RM Hybrid) when compared to only bulk or exfoliated $\mathrm{MoS}_{2}$ (2D MoS ), in agreement with the previously shown examples. The high density of exposed redox active edge and pore sites on the composite surface provide a large number of active sites for charge storage, in the form of proton adsorption $\left(\mathrm{MoS}_{2}+\mathrm{xH}^{+}+\mathrm{xe} \mathrm{e}^{-} \leftarrow \rightarrow \mathrm{H}_{\mathrm{x}} \mathrm{MoS}_{2}\right)$, to occur. ${ }^{286}$ The peaks present in the curve in Figure 20e indicate that some Faradaic reactions are occurring, and these have been attributed to the adsorption/desorption of protons, as the electrolyte used was $\mathrm{H}_{2} \mathrm{SO}_{4}$, at the interface.

\subsection{Hybrid composite batteries}

\subsubsection{Lithium ion batteries}

Lithium ion batteries (LIB) are another key energy storage technology for which composites of 2D materials can produce large improvements in performance. LIBs are rechargeable energy storage devices which function by shuttling lithium ions between a negative electrode (anode) and a positive electrode (cathode). Unlike supercapacitors, which are of interest due to rapid charge/discharge times, LIBs are able to provide a sustained current output over a long time (i.e. high energy density) but suffer from fatigue with repeated use. The typical anode in commercial LIBs consists of a graphite electrode, however graphite anodes suffer from a low theoretical capacity (372 $\mathrm{mAh} / \mathrm{g}$ ) as well as susceptibility to structural damage with continued lithium intercalation/de-intercalation with repeated charge/discharge cycle. ${ }^{288}$ Graphene has already begun to attract attention for use in LIBs due to the increased performance that occurs when reduced to nanoscale dimensions, which produces increased capacity (600-1000 $\mathrm{mAh} / \mathrm{g}$ ) and mechanical flexibility to resist deformation. ${ }^{289,290}$ Interest has also been extended to TMDs, where higher capacities ( $670 \mathrm{mAh} / \mathrm{g}$ ) have been measured. ${ }^{291-293}$ Sulphide containing materials are of particular interest due to the difference in the lithium storage mechanism, unlike graphite where the ions are simply intercalated between the carbon layers, the majority of intercalated lithium is stored through a conversion reaction with the sulphide, forming $\mathrm{Li}_{2} \mathrm{~S}$. This allows between 2-6 electrons per single transition metal compared to only a single electron for a simple intercalation reaction. ${ }^{294}$ As with supercapacitors there is also increasing attention focused on improving the performance (up to $1675 \mathrm{mAh} / \mathrm{g}$ ) through the creation of graphene/ $\mathrm{MoS}_{2}$ hybrid composites. ${ }^{250,263,264,266,295}$ By creating these hybrid composites of nanoscale 2D materials it is possible to achieve the desired high capacity along with long-term cycling stability. As with the other nanostructured composites containing graphene, the carbon can provide a highly conductive network providing both a conductive pathway within the composite, along with a physical prevention of material re-agglomeration and restacking.

One method for achieving maximum surface area, while preserving the close interaction between the composite materials, is through direct growth, typically via hydrothermal chemistry, of $\mathrm{MoS}_{2}$ onto a graphene support. ${ }^{267-272}$ By maximising the connectivity between the different materials that make up the composite it is possible to ensure rapid charge transfer kinetics as well as structural stability to compensate for the volume changes that occur when the lithium ions are intercalated. By creating a graphene aerogel structure that acts as both a highly conductive and flexible backbone as well as a porous structural support which can be 'decorated' with $\mathrm{MoS}_{2}$ it is possible to create a highly efficient LIB electrode. This has been shown by Lee et al., where chemically synthesised $\mathrm{MoS}_{2}$ is added to a dispersion of GO before hydrothermal treatment $\left(80{ }^{\circ} \mathrm{C}\right.$ for $\left.6 \mathrm{~h}\right)$ to form a hydrogel, which is then dried and compressed to form an aerogel paper, shown schematically in Figure 21a. ${ }^{269}$ The $\mathrm{MoS}_{2}$ chemical synthesis process, instead of forming stacked laminar sheets leads to the growth of 'nanoflowers' and the introduction of these into the GO structure leads to the formation of pores that are ideal for lithium intercalation. The electrochemical performance of this hybrid structure, which can be seen in Figure 21b, shows the high capacity of the electrodes even at high current density and illustrates again how the composite material $\left(\mathrm{MoS}_{2}\right.$ Graphene Aerogel Paper, MGAP) outperformed both the pure $\mathrm{MoS}_{2}$ as well as the simple mixture of the $\mathrm{MoS}_{2}$ and $\mathrm{GO}$ without the hydrothermal treatment (MrGO) at low current density. As the current density was increased the other composites produce higher capacitance, however, with repeated cycling this was found to decrease significantly, while the MGAP composite retained its performance. SEM images show the morphology of the compressed aerogel paper electrode are shown in Figure $21 c, d$, and a photograph illustrating the flexibility of the paper electrode material in Figure 21e, indicating its applicability for flexible energy storage devices.

In the case of GO there is typically a negative surface charge due to the presence of oxygen functional groups on the surface and this prevents the use of typical molybdenum oxide ions $\left(\mathrm{MoO}_{4}{ }^{2-}\right)$ being used to couple to the surface due to 
electrostatic repulsion. To overcome this and ensure that $\mathrm{MoS}_{2}$ can be easily directly grown onto $\mathrm{GO}$ while remaining in an aqueous dispersion several researchers have attempted to functionalise the GO using cationic surfactants to neutralise its surface charge. ${ }^{247,296}$ As the surface charge on GO is strongly affected by the $\mathrm{pH}$ it is also possible to tune this charge by altering the acidity of the dispersion. By carefully controlling the $\mathrm{pH}$ it was demonstrated by Teng et al. that it is possible to selectively grow $\mathrm{MoS}_{2}$ nanosheets on $\mathrm{GO}$ using hydrothermal synthesis, leading to vertically aligned $\mathrm{MoS}_{2}$ sheets for use in LIBs. ${ }^{271}$ In this work, shown schematically in Figure 21f, the $\mathrm{MoS}_{2}$ nanosheets are directly coupled to the oxygen atoms present on the surface of the $\mathrm{GO}$ by hydrothermal synthesis. This leads to perpendicular alignment of the $\mathrm{MoS}_{2}$ which helps to prevent restacking of the composite structure, thereby maximising available surface area, as well as ensuring high structural stability during the cycling process. The GO backbone structure provides a conductive pathway for charge and lowers the diffusion distance required for the electrolyte ions. Similar to previously discussed work (section 4.3), the $\mathrm{MoS}_{2}$ sheets here are significantly smaller in diameter (100 $\mathrm{nm}$ ) than the GO sheets, and this small size is preferential due to the shorter diffusion lengths required for the lithium ions as well as promoting the electrode/electrolyte interface. The cycling performance of this $\mathrm{MoS}_{2}$ /graphene electrode is shown in Figure 21g, along with comparison to pure $\mathrm{MoS}_{2}$ and $\mathrm{rGO}$ electrodes. The initial capacity of the composite electrode is higher than either of the singular component electrodes (1160 $\mathrm{mAh} / \mathrm{g}$ ), and during initial cycling the composite and pure $\mathrm{MoS}_{2}$ electrodes undergo an increase in capacity. This is attributed to an increase in the available surface sites with repeated cycling, and matches what has been observed previously for supercapacitor devices. After $\sim 20$ cycles the pure $\mathrm{MoS}_{2}$ electrode undergoes a rapid decline in capacity, due to the combination of its low conductivity and inability to withstand the repeated volume changes. The composite however displays negligible capacity loss over 150 cycles, indicating the greatly enhanced stability and capacity of an in situ grown $\mathrm{MoS}_{2}$ /graphene composite.

\subsubsection{Sodium ion batteries}

Additional to the well-studied $\mathrm{MoS}_{2}$ /graphene Li-ion systems there has also be a recent increase in interest in sodium ion battery (SIB) systems using these hybrid composites. ${ }^{244,267,297}$ This increased interest is driven by the relative abundance combined with worldwide distribution of sodium reserves. Sodium intercalation chemistry is also very similar to lithium, making it simple to transition to as an alternative material. However, as the radius of $\mathrm{Na}^{+}$ions are $\sim 55 \%$ larger than $\mathrm{Li}^{+}$this means electrode materials must be able to withstand much greater physical deformation during charge/discharge cycles. An example of one $\mathrm{MoS}_{2} / \mathrm{GO}$ composite paper film for use in SIBs, where bulk $\mathrm{MoS}_{2}$ powder was first exfoliated using ultrasonication in acidic media and combined with a GO dispersion, is shown in Figure $22 a, b .^{244}$ This procedure, similar to the others discussed, produces a laminar structure where the large GO sheets are interleaved with smaller $\mathrm{MoS}_{2}$ flakes, providing a robust electrode architecture ideal for reversible storage of the large sodium ions, as shown schematically in Figure 22c. The incorporation of GO into this composite was found to have a non-linear effect on the measured conductivity of the resultant composite due to the established percolation theory that describes the formation of a single conductive pathway that can form when a conductive additive is inserted into an insulating matrix. ${ }^{244,298}$ The $1^{\text {st }}$ and $2^{\text {nd }}$ cycles of sodiation and de-sodiation are shown in Figure 22d for a $60 \% \mathrm{MoS}_{2}$ /graphene composite reaching a capacity of 230 $\mathrm{mAhg}^{-1}$ and a Coulombic efficiency of $99 \%$. The high irreversible capacity observed during the first cycle is attributed to the formation of the solid electrolyte interphase (SEI) layer typically observed in such battery systems. As the ratio of $\mathrm{MoS}_{2}$ exceeded $40 \%$ the electrochemical behaviour was dominated by the sodium interaction with $\mathrm{MoS}_{2}$ over that of the GO, indicating the ratio of each material in these composites is of importance. The mechanical strength of these composites were also investigated, as $2 \mathrm{D}$ materials are of great interest for flexible energy storage due to their flexibility, ${ }^{299}$ and the composite was found to be able to withstand 2-4 MPa of uniaxial tension. The electrochemical intercalation and deintercalation of sodium ions that occur during charge/discharge cycles also lead to phase transitions of the $\mathrm{MoS}_{2}$, as seen previously in the case of lithium, due to charge transfer from the sodium ions, as shown schematically in Figure 22e. This has been investigated, on the atomic-scale, to better understand this transition and was shown to occur in discrete stages where depending on the degree of intercalation the transition is reversible. ${ }^{300}$ During the charge cycle the sodium ions first intercalate into alternating layers of the $\mathrm{MoS}_{2}$, as all the layers are intercalated the large degree of strain on the lattice induces a glide of the sulphur atoms along the interlayer atomic plane causing the phase transition from $2 \mathrm{H}$-to- $1 \mathrm{~T}^{287,300}$ The partially intercalated $\mathrm{MoS}_{2}$ layers, with distinct combinations of both $1 \mathrm{~T}$ and $2 \mathrm{H}$ regions are shown in Figure 22f, along with a cross-section in Figure 22g showing the intercalated sodium ions (blue circles). Properly understanding the structural changes that occur within the $\mathrm{MoS}_{2}$ layers when intercalation occurs is important for the design and optimisation of composite devices for use in energy storage.

\subsection{Other TMDs for energy applications}

As discussed above so far the vast majority of hybrid composites that are present in the literature are combinations of $\mathrm{MoS}_{2}$ and graphene derivatives (graphene, GO and rGO). However, other TMDs have also been investigated for use in hybrid composites for applications in energy storage. Each of the most commonly used TMDs has previously been compared for their energy storage performance and due to differences in density, electronic structure, toxicity, as well as ease of production each are suited to different applications. ${ }^{256,301} \mathrm{An}$ example of the different capacitive performance for several TMDs, without the use of any carbon additives is shown in 
Figure 23a-c. ${ }^{256}$ The different TMDs investigated were $\mathrm{MoS}_{2}$, $\mathrm{WS}_{2}, \mathrm{TiS}_{2}$, and $\mathrm{MoSe}_{2}$ (crystal structures shown in Figure 23a) and each were prepared by ultrasonication. A photograph of the dispersions is shown in Figure 23b. From the comparison of the capacitive performance (Figure 23c) the best performing material was $\mathrm{TiS}_{2}$, which can be attributed to its metallic electronic structure. The use of $\mathrm{TiS}_{2}$ compared to other TMDs has already been demonstrated to provide improved performance for energy storage applications. ${ }^{302}$ This demonstrates that depending on the particular application careful thought must be given to which TMD will work best. Hybrid graphene-based composites of different TMDs have also been recently investigated, with $\mathrm{WS}_{2} / \mathrm{rGO}^{248}$ as well as $\mathrm{MoSe}_{2} / \mathrm{rGO}^{303}$ composites each produced from hydrothermal synthesis showing improved capacitive performance (Figure $23 d, e)$. The superior performance of $\mathrm{WS}_{2} /$ graphene ${ }^{304}$ and $\mathrm{MoSe}_{2} /$ graphene ${ }^{305}$ composites for LIB applications are also shown in Figure 23f,g, showing that as well as increased energy storage these composites provide improvements in cycle stability and device lifetimes. Each of these examples shows the universality of the improved performance that occurs when hybrid graphene based composites are compared to single component materials. The addition of a highly conductive yet flexible network is ideal for these applications. The use of in situ growth processes has also shown to provide improved performance for different material combinations as the strengthened interaction between the different materials leads to increased charge transfer and mechanical robustness.

\subsection{Phosphorene/graphene hybrid composites}

Recently some groups have begun to investigate graphene based composites with more exotic layered 2D materials, and one example of this that shows particular promise for energy storage applications is graphene/phosphorene composites. Phosphorene, which is exfoliated black phosphorous (BP), has already been demonstrated to be suitable for the same scalable liquid based exfoliation techniques that are applicable for TMDs. ${ }^{306-308}$ Phosphorene possesses high p-type carrier mobility ( $>1000 \mathrm{~cm}^{2} \mathrm{~V}^{-1} \mathrm{~s}^{-1}$ ) and a tuneable direct band gap in the range of $0.3-2 \mathrm{eV}$ making it of great interest for many electronic applications. ${ }^{308}$ The layered structure of phosphorene can be seen in Figure 24a along with an SEM image of bulk BP before exfoliation. By dispersing this BP in suitable solvents, such as $n$-methylpyrrolidone (NMP), it is possible to create stable dispersions (shown in Figure 24b) of few-layer BP, which is often referred to as phosphorene. TEM images of the structure of these exfoliated few-layer phosphorene flakes (Figure 24c-f) show the ordered lattice array. ${ }^{309}$ Typically phosphorene is considered highly unstable in air and degrades quickly; however, the process of solution exfoliation in NMP leads to a coating of residual solvent molecules which protect the phosphorene flakes from oxidation. ${ }^{308}$ This allows the solution exfoliated phosphorene flakes to be used in a much larger range of applications, including when exposed to aqueous solutions. This solution exfoliated phosphorene can also be combined with GO dispersions and used to create hybrid composite papers through vacuum filtration, such as seen in Figure $24 \mathrm{~g} .{ }^{310}$ To produce these papers dispersions of $\mathrm{GO}$ and solution exfoliated BP were combined and then filtered to produce the thin, flexible membrane. This membrane was subsequently thermally reduced $\left(300{ }^{\circ} \mathrm{C}\right.$ for $\left.1 \mathrm{~h}\right)$ to produce an $\mathrm{rGO} /$ phosphorene composite (PG) and also compared to a membrane that underwent spark plasma sintering $\left(300{ }^{\circ} \mathrm{C}, 10\right.$ $\mathrm{MPa}$ ) (PG-SPS). The cross-section SEM images (Figure $24 \mathrm{~h}-\mathrm{i}$ ) show that the PG-SPS sample is significantly denser than the thermally reduced sample (PG), attributed to a reduction in oxygen and water content. These phosphorene/graphene composites were then characterised for use in LIBs and the measured specific capacity, with increasing current densities, for both membranes is shown in Figure 24j. The PG-SPS electrode displayed a high reversible specific capacity (1302 $\mathrm{mAhg}^{-1}$ ) and displayed superior cycle lifetime illustrating the enhanced performance of these phosphorene based composite.

Phosphorene/graphene composites can also be used for SIBs and a schematic showing the process of sodium intercalation (sodiation) and de-intercalation (desodiation), where the sodium ions attach directly to the phosphorene flakes is shown in Figure $24 \mathrm{k}$. Phosphorus can react with sodium and lithium form $\mathrm{Na}_{3} \mathrm{P}$ and $\mathrm{Li}_{3} \mathrm{P}$ respectively, with a high theoretical specific capacity of $2,596 \mathrm{~mA} \mathrm{~h} \mathrm{~g}^{-1}$, which is significantly larger than other SIB materials currently available. ${ }^{311}$ Phosphorene/graphene composites created by mixing ultrasonicated dispersions of both graphene and phosphorene in NMP, were characterised during electrochemical sodium intercalation by Sun et al. ${ }^{311}$ During sodiation there was a significant volume expansion ( 160\%) and to ensure long cycle lifetime the use of smaller phosphorene flakes relative to the size of the graphene, similar to the results seen from the TMDs discussed previously, was suggested to be key. This provides an elastic 'buffer' space in between the small phosphorene flakes that are sandwiched between larger graphene flakes, this also minimises diffusion length for both electrons and sodium ions. The relative electrochemical performance for different ratio graphene/phosphorene composites is shown in Figure 24l, where the ratio between the graphene and phosphorene is presented as a carbon/phosphorous (C/P) ratio. The charge/discharge cycling showed that composites with a C/P ratio of 2.78:1 (blue line) and 3.46:1 (green line) maintained the highest capacity after 100 cycles. As the graphene by itself is electrochemically inactive for sodium the lower $C / P$ ratios also provide the highest measured capacities. This work and the previous example both demonstrate that hybrid composites of phosphorene/graphene are promising candidates for future studies into energy storage. The graphene provides elastic, highly conductive buffer layers that can allow for large volume expansion while maintaining a preferential pathway for electrons generated during charge/discharge allowing the capacity of the phosphorene to be exploited. 


\subsection{Hybrid composites for catalytic hydrogen evolution}

As well as energy storage technologies, such as supercapacitors and batteries, hybrid composites of 2D materials are ideal for catalytic energy production, such as catalytic hydrogen evolution (HER) reactions. ${ }^{312}$ Electrocatalytic production of hydrogen, through splitting of water, is a vitally important component of renewable energy. An ideal catalyst for HER should minimise the required overpotential required for hydrogen production, and the best materials for this currently are the platinum group metals. The volcano plot showing the relative catalytic performance of a variety of noble metals, as well as TMDs and graphene/TMD composites is shown in Figure $25 a$, where $\Delta G_{H}$ and $j_{0}$ are the Gibbs free energy of hydrogen evolution and current density respectively. The performance of the TMDs can be seen to approach that of the far more expensive noble metals (Pt \& $\mathrm{Pd}$ ). $\mathrm{MoS}_{2}$ in particular has been investigated for catalytic HER previously due to calculations and experimental results which show that the active sites on the $\mathrm{MoS}_{2}$ are the edges while the basal plane remains catalytically inert. ${ }^{239,313-315}$ Thus, exfoliated nanosheets of $\mathrm{MoS}_{2}$ are an ideal catalyst for this application due to the high density of edge sites.

Hybrid composites consisting of nanostructured $\mathrm{MoS}_{2}$ supported on conductive graphene based materials provide performance benefits due to the utilization of the high specific surface area combined with electrical conductance of the supporting material. Such hybrid composites where rGO sheets have been decorated with $\mathrm{MoS}_{2}$ created through a onestep solvothermal reaction in dimethylformamide have been demonstrated previously. ${ }^{316}$ During this process a precursor $\left(\left(\mathrm{NH}_{4}\right)_{2} \mathrm{MoS}_{4}\right)$ was reduced to form $\mathrm{MoS}_{2}$ on the surface of the $\mathrm{GO}$, while reducing the $\mathrm{GO}$ to form $\mathrm{rGO}$ by reacting with hydrazine. This procedure forms $\mathrm{rGO}$ sheets which are uniformly decorated with $\mathrm{MoS}_{2}$ nanoparticles, and it was observed that the $\mathrm{MoS}_{2}$ selectively formed in contact with the rGO sheets, with minimal formation of nanoparticles freely in solution. This mechanism behind this selective growth was attributed to the interaction between the oxygen functional groups present on the GO surface, in agreement with the previously discussed work, ${ }^{271}$ providing control over the heterostructure morphology. The catalytic performance of the individual components was poor while the hybrid composite displayed excellent catalytic activity, approaching that of theoretical maximum of platinum. This high catalytic performance was attributed to the strong electronic coupling between the small $\mathrm{MoS}_{2}$ nanosheets and $\mathrm{rGO}$ sheets, which maximises the availability of catalytically active edge sites, in agreement with previously observed increases when 2D materials are combined to form a synergistic composite structure.

The combination of metal nanoparticles into a composite $\mathrm{TMD} /$ graphene structure can also be used to further improve the catalytic efficiency, as demonstrated by Toth et al., and shown in Figure $25 \mathrm{~b}-\mathrm{d} .{ }^{317}$ To measure the catalytic activity and fundamental electrochemical properties of a graphene/ $\mathrm{MoS}_{2}$ heterostructure a 'sandwich' was created at the interface

$20 \mid$ J. Name., 2012, 00, 1-3 between two immiscible liquids. This liquid/liquid interface has been widely used in electrochemistry to investigate the properties of a material free from the interference that may be caused by interaction with a solid substrate. ${ }^{318,319}$ This interface also allows for the formation of films of material to be studied at a highly reproducible, molecularly sharp, and defect free focal plane and this has been demonstrated for a wide range of particles with different shapes and aspect ratios. ${ }^{320}$ By creating a range of different composite structures, shown schematically in Figure 25b, using CVD grown graphene as a supporting layer combined with either vertically aligned CVD grown $\mathrm{MoS}_{2}\left(\mathrm{VMoS}_{2}\right)$ or ultrasonication liquid exfoliated $\mathrm{MoS}_{2}\left(\mathrm{eMoS}_{2}\right)$ it was possible to investigate the behaviour of these composites at the liquid/liquid interface. This $\mathrm{MoS}_{2}$ /graphene sandwich could then be decorated with metal nanoparticles ( $\mathrm{Pd}, \mathrm{Pt}, \mathrm{Au}$ or $\mathrm{Ag}$ ) that were grown directly onto the heterostructure at the liquid interface by reducing metal salts present in the electrolyte solutions. This asymmetrical decoration, or Janus functionalization, can be used to tune the properties of different $2 \mathrm{D}$ materials and change their properties. ${ }^{321}$ The catalytic performance of these 'sandwiches' are shown in Figure 25c where the catalytic performance of the $\mathrm{MoS}_{2}$ /graphene composite is again greatly increased over the single component films, this can be enhanced further by the deposition of these metal nanoparticles. The most active composite structure was the vertically aligned CVD grown $\mathrm{MoS}_{2}$, due to the high density of exposed edge sites, combined with graphene and palladium nanoparticles $\left(\mathrm{vMoS}_{2} / \mathrm{Gr} / \mathrm{Pd}\right.$, shown in green). The capacitance of these composite 'sandwich' films was also measured, after transferring them onto a supporting substrate, shown in Figure $25 \mathrm{~d}$-e, again the $\mathrm{vMoS}_{2} / \mathrm{Gr} / \mathrm{Pd}$ composite exhibited superior performance. This increased capacitance was attributed to a high available surface area, and the ability to provide large amounts of active surface sites for the electrolyte ions $\left(\mathrm{Li}^{+}\right)$to adsorb/intercalate into the $\mathrm{MoS}_{2}$ surface. This work demonstrates a unique architecture to characterise the fundamental electrochemical behaviour of $\mathrm{MoS}_{2} /$ graphene heterostructure composites for both HER and capacitive energy storage.

\section{Summary}

In this review, we have overviewed the research on the graphenebased heterostructures which are prepared either by dry methods, such as mechanical stacking and CVD, or by wet methods, such as liquid-phase exfoliation and mixing processes. These heterostructures are composed of graphene with other 2D materials or with lower dimensionality materials, which are stacked, connected or intercalated in graphene material. Integration into heterostructure give us a new opportunity to bring out new functionalities due to synergetic effects which can be enhanced by van der Waals interaction. The integration also allows to overcome the drawbacks of graphene, such as the lack of a bandgap, and of other 2D materials, such as the low mobility of TMDs. Even though research of such heterostructured materials mainly started at the beginning of this decade, advances have been quite fast, relying on the accumulated knowledge on the synthesis and handling of $2 \mathrm{D}$ 
materials. These advances have allowed to fabricate almost any kind of heterostructure at the laboratory scale, with prototypes showing functionalities than in some cases approximate or even exceed those of current technologies. Also, there is still space for new 2D layered materials to emerge, which will definitely enrich the field of heterostructures. Finally, tailored physical properties and designed geometrical structures may be obtained by an appropriate selection of the preparation methods and materials. With further development of the production methods of graphene and other 2D materials as well as their heterostructures, advances are expected to develop these unique materials into practical applications in a wide variety of fields, including electronics, photonics, sensing technologies and production and storage of energy.

\section{Acknowledgements}

This work was supported by the PRESTO-JST and JSPS KAKENHI Grant Numbers JP15H03530, JP15K13304, JP16H00917. 


\section{References}

1 A. C. Ferrari, F. Bonaccorso, V. Fal'ko, K. S. Novoselov, S. Roche, P. Bøggild, S. Borini, F. H. L. Koppens, V. Palermo, N. Pugno, J. A. Garrido, R. Sordan, A. Bianco, L. Ballerini, M. Prato, E. Lidorikis, J. Kivioja, C. Marinelli, T. Ryhänen, A. Morpurgo, J. N. Coleman, V. Nicolosi, L. Colombo, A. Fert, M. Garcia-Hernandez, A. Bachtold, G. F. Schneider, F. Guinea, C. Dekker, M. Barbone, Z. Sun, C. Galiotis, A. N. Grigorenko, G. Konstantatos, A. Kis, M. Katsnelson, L. Vandersypen, A. Loiseau, V. Morandi, D. Neumaier, E. Treossi, V. Pellegrini, M. Polini, A. Tredicucci, G. M. Williams, B. H. Hong, J.-H. Ahn, J. M. Kim, H. Zirath, B. J. van Wees, $H$. van der Zant, $L$. Occhipinti, A. D. Matteo, I. A. Kinloch, T. Seyller, E. Quesnel, X. Feng, K. Teo, N. Rupesinghe, P. Hakonen, S. R. T. Neil, Q. Tannock, T. Löfwander and J. Kinaret, Nanoscale, 2015, 7, 4598-4810.

2 K. S. Novoselov, A. K. Geim, S. V. Morozov, D. Jiang, Y. Zhang, S. V. Dubonos, I. V. Grigorieva and A. A. Firsov, Science, 2004, 306, 666-669.

3 C. Berger, Z. Song, T. Li, X. Li, A. Y. Ogbazghi, R. Feng, Z. Dai, A. N. Marchenkov, E. H. Conrad, P. N. First and W. A. de Heer, J. Phys. Chem. B, 2004, 108, 19912-19916.

4 Y. Zhang, L. Zhang and C. Zhou, Acc. Chem. Res., 2013, 46, 2329-2339.

5 S. Stankovich, R. D. Piner, X. Chen, N. Wu, S. T. Nguyen and R. S. Ruoff, J. Mater. Chem., 2006, 16, 155-155.

6 J. I. Paredes, S. Villar-Rodil, P. Solís-Fernández, M. J. Fernández-Merino, L. Guardia, A. Martínez-Alonso and J. M. D. Tascón, J. Alloys Compd., 2012, 536, S450-S455.

7 K. S. Novoselov, D. Jiang, F. Schedin, T. J. Booth, V. V. Khotkevich, S. V. Morozov and A. K. Geim, Proc. Natl. Acad. Sci., 2005, 102, 10451-10453.

8 C. R. Dean, A. F. Young, I. Meric, C. Lee, L. Wang, S. Sorgenfrei, K. Watanabe, T. Taniguchi, P. Kim, K. L. Shepard and J. Hone, Nat. Nanotechnol., 2010, 5, 722-726.

9 B. Radisavljevic, A. Radenovic, J. Brivio, V. Giacometti and A. Kis, Nat. Nanotechnol., 2011, 6, 147-150.

10 A. K. Geim and I. V. Grigorieva, Nature, 2013, 499, 419-425.

11 R. Kitaura, Y. Miyata, R. Xiang, J. Hone, J. Kong, R. S. Ruoff and S. Maruyama, J. Phys. Soc. Jpn., 2015, 84, 121013.

12 G. R. Bhimanapati, Z. Lin, V. Meunier, Y. Jung, J. Cha, S. Das, D. Xiao, Y. Son, M. S. Strano, V. R. Cooper, L. Liang, S. G. Louie, E. Ringe, W. Zhou, S. S. Kim, R. R. Naik, B. G. Sumpter, H. Terrones, F. Xia, Y. Wang, J. Zhu, D. Akinwande, N. Alem, J. A. Schuller, R. E. Schaak, M. Terrones and J. A. Robinson, ACS Nano, 2015, 9, 11509-11539.

13 M. Nasilowski, B. Mahler, E. Lhuillier, S. Ithurria and B. Dubertret, Chem. Rev., 2016, 116, 10934-10982.

14 V. Nicolosi, M. Chhowalla, M. G. Kanatzidis, M. S. Strano and J. N. Coleman, Science, 2013, 340, 1226419.

15 M. Xu, T. Liang, M. Shi and H. Chen, Chem. Rev., 2013, 113, 3766-3798.

16 P. Miró, M. Audiffred and T. Heine, Chem. Soc. Rev., 2014, 43, 6537-6554.

17 H. Liu, A. T. Neal, Z. Zhu, Z. Luo, X. Xu, D. Tománek and P. D. Ye, ACS Nano, 2014, 8, 4033-4041.

18 W. Ge, K. Kawahara, M. Tsuji and H. Ago, Nanoscale, 2013, 5, 5773-5778.

19 A. Ambrosi, Z. Sofer and M. Pumera, Chem. Commun., 2015, 51, 8450-8453.

20 D. K. Efetov, L. Wang, C. Handschin, K. B. Efetov, J. Shuang, R. Cava, T. Taniguchi, K. Watanabe, J. Hone, C. R. Dean and P. Kim, Nat. Phys., 2015, 12, 328-332.

21 C.-L. Song, Y.-L. Wang, Y.-P. Jiang, Z. Li, L. Wang, K. He, X. Chen, X.-C. Ma and Q.-K. Xue, Phys. Rev. B, 2011, 84, 20503.
22 D. Jiang, T. Hu, L. You, Q. Li, A. Li, H. Wang, G. Mu, Z. Chen, H. Zhang, G. Yu, J. Zhu, Q. Sun, C. Lin, H. Xiao, X. Xie and M. Jiang, Nat. Commun., 2014, 5, 5708.

23 H. Sahabudeen, H. Qi, B. A. Glatz, D. Tranca, R. Dong, Y. Hou, T. Zhang, C. Kuttner, T. Lehnert, G. Seifert, U. Kaiser, A. Fery, Z. Zheng and X. Feng, Nat. Commun., 2016, 7, 13461.

24 A. Turchanin and A. Gölzhäuser, Adv. Mater., 2016, 28, 6075-6103.

25 C. Tan and H. Zhang, Nat. Commun., 2015, 6, 7873.

26 K.-A. N. Duerloo, Y. Li and E. J. Reed, Nat. Commun., 2014, 5, 4214.

27 L. Britnell, R. M. Ribeiro, A. Eckmann, R. Jalil, B. D. Belle, A. Mishchenko, Y.-J. Kim, R. V. Gorbachev, T. Georgiou, S. V. Morozov, A. N. Grigorenko, A. K. Geim, C. Casiraghi, A. H. C. Neto and K. S. Novoselov, Science, 2013, 340, 1311-1314.

28 Y. Liu, N. O. Weiss, X. Duan, H.-C. Cheng, Y. Huang and X. Duan, Nat. Rev. Mater., 2016, 1, 16042.

29 F. Bonaccorso, Z. Sun, T. Hasan and A. C. Ferrari, Nat. Photonics, 2010, 4, 611-622.

30 M. P. Levendorf, C.-J. Kim, L. Brown, P. Y. Huang, R. W. Havener, D. A. Muller and J. Park, Nature, 2012, 488, 627632.

31 G. Fiori, A. Betti, S. Bruzzone and G. lannaccone, ACS Nano, 2012, 6, 2642-2648.

32 Z. Liu, L. Ma, G. Shi, W. Zhou, Y. Gong, S. Lei, X. Yang, J. Zhang, J. Yu, K. P. Hackenberg, A. Babakhani, J.-C. Idrobo, R. Vajtai, J. Lou and P. M. Ajayan, Nat. Nanotechnol., 2013, 8, 119-124.

33 Y. Gao, Y. Zhang, P. Chen, Y. Li, M. Liu, T. Gao, D. Ma, Y. Chen, Z. Cheng, X. Qiu, W. Duan and Z. Liu, Nano Lett., 2013, 13, 3439-3443.

34 H.-C. Shin, Y. Jang, T.-H. Kim, J.-H. Lee, D.-H. Oh, S. J. Ahn, J. H. Lee, Y. Moon, J.-H. Park, S. J. Yoo, C.-Y. Park, D. Whang, C.W. Yang and J. R. Ahn, J. Am. Chem. Soc., 2015, 137, 68976905.

35 Z. Liu, L. H. G. Tizei, Y. Sato, Y.-C. Lin, C.-H. Yeh, P.-W. Chiu, M. Terauchi, S. lijima and K. Suenaga, Small, 2016, 12, 252259.

36 A. S. Mayorov, R. V. Gorbachev, S. V. Morozov, L. Britnell, R. Jalil, L. A. Ponomarenko, P. Blake, K. S. Novoselov, K. Watanabe, T. Taniguchi and A. K. Geim, Nano Lett., 2011, 11, 2396-2399.

37 L. A. Ponomarenko, A. K. Geim, A. A. Zhukov, R. Jalil, S. V. Morozov, K. S. Novoselov, I. V. Grigorieva, E. H. Hill, V. V. Cheianov, V. I. Fal'ko, K. Watanabe, T. Taniguchi and R. V. Gorbachev, Nat. Phys., 2011, 7, 958-961.

38 H. Wang, T. Taychatanapat, A. Hsu, K. Watanabe, T. Taniguchi, P. Jarillo-Herrero and T. Palacios, IEEE Electron Device Lett., 2011, 32, 1209-1211.

39 C. T. Nottbohm, A. Turchanin, A. Beyer, R. Stosch and A. Gölzhäuser, Small, 2011, 7, 874-883.

40 L. Britnell, R. V. Gorbachev, R. Jalil, B. D. Belle, F. Schedin, A. Mishchenko, T. Georgiou, M. I. Katsnelson, L. Eaves, S. V. Morozov, N. M. R. Peres, J. Leist, A. K. Geim, K. S. Novoselov and L. A. Ponomarenko, Science, 2012, 335, 947-950.

41 S. J. Haigh, A. Gholinia, R. Jalil, S. Romani, L. Britnell, D. C. Elias, K. S. Novoselov, L. A. Ponomarenko, A. K. Geim and R. Gorbachev, Nat. Mater., 2012, 11, 764-767.

42 Y.-C. Lin, C.-C. Lu, C.-H. Yeh, C. Jin, K. Suenaga and P.-W. Chiu, Nano Lett., 2012, 12, 414-419.

43 T. Uwanno, Y. Hattori, T. Taniguchi, K. Watanabe and K. Nagashio, 2D Mater., 2015, 2, 41002.

44 A. V. Kretinin, Y. Cao, J. S. Tu, G. L. Yu, R. Jalil, K. S. Novoselov, S. J. Haigh, A. Gholinia, A. Mishchenko, M. Lozada, T. Georgiou, C. R. Woods, F. Withers, P. Blake, G. Eda, A. Wirsig, C. Hucho, K. Watanabe, T. Taniguchi, A. K. Geim and R. V. Gorbachev, Nano Lett., 2014, 14, 3270-3276. 
45 L. Wang, I. Meric, P. Y. Huang, Q. Gao, Y. Gao, H. Tran, T. Taniguchi, K. Watanabe, L. M. Campos, D. A. Muller, J. Guo, P. Kim, J. Hone, K. L. Shepard and C. R. Dean, Science, 2013, 342, 614-617.

46 A. Castellanos-Gomez, M. Buscema, R. Molenaar, V. Singh, L. Janssen, H. S. J. van der Zant and G. A. Steele, 2D Mater., 2014, 1, 11002.

47 P. J. Zomer, M. H. D. Guimarães, J. C. Brant, N. Tombros and B. J. van Wees, Appl. Phys. Lett., 2014, 105, 13101.

48 M. H. Gass, U. Bangert, A. L. Bleloch, P. Wang, R. R. Nair and A. K. Geim, Nat. Nanotechnol., 2008, 3, 676-681.

49 Y. Cao, A. Mishchenko, G. L. Yu, E. Khestanova, A. P. Rooney, E. Prestat, A. V. Kretinin, P. Blake, M. B. Shalom, C. Woods, J. Chapman, G. Balakrishnan, I. V. Grigorieva, K. S. Novoselov, B. A. Piot, M. Potemski, K. Watanabe, T. Taniguchi, S. J. Haigh, A. K. Geim and R. V. Gorbachev, Nano Lett., 2015, 15, 4914-4921.

50 K. Kim, M. Yankowitz, B. Fallahazad, S. Kang, H. C. P. Movva, S. Huang, S. Larentis, C. M. Corbet, T. Taniguchi, K. Watanabe, S. K. Banerjee, B. J. LeRoy and E. Tutuc, Nano Lett., 2016, 16, 1989-1995.

51 C. R. Woods, F. Withers, M. J. Zhu, Y. Cao, G. Yu, A. Kozikov, M. B. Shalom, S. V. Morozov, M. M. van Wijk, A. Fasolino, M. I. Katsnelson, K. Watanabe, T. Taniguchi, A. K. Geim, A. Mishchenko and K. S. Novoselov, Nat. Commun., 2016, 7, 10800.

52 D. Wang, G. Chen, C. Li, M. Cheng, W. Yang, S. Wu, G. Xie, J. Zhang, J. Zhao, X. Lu, P. Chen, G. Wang, J. Meng, J. Tang, R. Yang, C. He, D. Liu, D. Shi, K. Watanabe, T. Taniguchi, J. Feng, Y. Zhang and G. Zhang, Phys. Rev. Lett., 2016, 116, 126101.

53 M. Zhu, D. Ghazaryan, S.-K. Son, C. R. Woods, A. Misra, L. He, Takashi Taniguchi, K. Watanabe, K. S. Novoselov, Y. Cao and A. Mishchenko, 2D Mater., 2017, 4, 11013.

54 S. B. Desai, S. R. Madhvapathy, M. Amani, D. Kiriya, M. Hettick, M. Tosun, Y. Zhou, M. Dubey, J. W. Ager, D. Chrzan and A. Javey, Adv. Mater., 2016, 28, 4053-4058.

55 K. Roy, M. Padmanabhan, S. Goswami, T. P. Sai, G. Ramalingam, S. Raghavan and A. Ghosh, Nat. Nanotechnol., 2013, 8, 826-830.

56 S. Bertolazzi, D. Krasnozhon and A. Kis, ACS Nano, 2013, 7, 3246-3252.

57 W. J. Yu, Z. Li, H. Zhou, Y. Chen, Y. Wang, Y. Huang and X. Duan, Nat. Mater., 2013, 12, 246-252.

58 W. J. Yu, Y. Liu, H. Zhou, A. Yin, Z. Li, Y. Huang and X. Duan, Nat. Nanotechnol., 2013, 8, 952-958.

59 S. Larentis, J. R. Tolsma, B. Fallahazad, D. C. Dillen, K. Kim, A. H. MacDonald and E. Tutuc, Nano Lett., 2014, 14, 20392045.

60 W. Zhang, C.-P. Chuu, J.-K. Huang, C.-H. Chen, M.-L. Tsai, Y.H. Chang, C.-T. Liang, Y.-Z. Chen, Y.-L. Chueh, J.-H. He, M.-Y. Chou and L.-J. Li, Sci. Rep., 2014, 4, 3826.

61 P. T. K. Loan, W. Zhang, C.-T. Lin, K.-H. Wei, L.-J. Li and C.-H. Chen, Adv. Mater., 2014, 26, 4838-4844.

62 D. Pierucci, H. Henck, C. H. Naylor, H. Sediri, E. Lhuillier, A. Balan, J. E. Rault, Y. J. Dappe, F. Bertran, P. L. Fèvre, A. T. C. Johnson and A. Ouerghi, Sci. Rep., 2016, 6, 26656.

63 T. Georgiou, R. Jalil, B. D. Belle, L. Britnell, R. V. Gorbachev, S. V. Morozov, Y.-J. Kim, A. Gholinia, S. J. Haigh, O. Makarovsky, L. Eaves, L. A. Ponomarenko, A. K. Geim, K. S. Novoselov and A. Mishchenko, Nat. Nanotechnol., 2013, 8, 100-103.

64 P. J. Zomer, S. P. Dash, N. Tombros and B. J. van Wees, Appl. Phys. Lett., 2011, 99, 232104.

65 M. S. Choi, G.-H. Lee, Y.-J. Yu, D.-Y. Lee, S. Hwan Lee, P. Kim, J. Hone and W. Jong Yoo, Nat. Commun., 2013, 4, 1624.

66 G.-H. Lee, Y.-J. Yu, X. Cui, N. Petrone, C.-H. Lee, M. S. Choi, D.-Y. Lee, C. Lee, W. J. Yoo, K. Watanabe, T. Taniguchi, C. Nuckolls, P. Kim and J. Hone, ACS Nano, 2013, 7, 7931-7936.
67 L. Banszerus, M. Schmitz, S. Engels, M. Goldsche, K. Watanabe, T. Taniguchi, B. Beschoten and C. Stampfer, Nano Lett., 2016, 16, 1387-1391.

68 Z. Liu, L. Song, S. Zhao, J. Huang, L. Ma, J. Zhang, J. Lou and P. M. Ajayan, Nano Lett., 2011, 11, 2032-2037.

69 Y.-C. Lin, N. Lu, N. Perea-Lopez, J. Li, Z. Lin, X. Peng, C. H. Lee, C. Sun, L. Calderin, P. N. Browning, M. S. Bresnehan, M. J. Kim, T. S. Mayer, M. Terrones and J. A. Robinson, ACS Nano, 2014, 8, 3715-3723.

70 C. Oshima, A. Itoh, E. Rokuta, T. Tanaka, K. Yamashita and T. Sakurai, Solid State Commun., 2000, 116, 37-40.

71 X. Ding, G. Ding, X. Xie, F. Huang and M. Jiang, Carbon, 2011, 49, 2522-2525.

72 J. M. Garcia, U. Wurstbauer, A. Levy, L. N. Pfeiffer, A. Pinczuk, A. S. Plaut, L. Wang, C. R. Dean, R. Buizza, A. M. Van Der Zande, J. Hone, K. Watanabe and T. Taniguchi, Solid State Commun., 2012, 152, 975-978.

73 S. Tang, G. Ding, X. Xie, J. Chen, C. Wang, X. Ding, F. Huang, W. Lu and M. Jiang, Carbon, 2012, 50, 329-331.

74 M. Wang, S. K. Jang, W.-J. Jang, M. Kim, S.-Y. Park, S.-W. Kim, S.-J. Kahng, J.-Y. Choi, R. S. Ruoff, Y. J. Song and S. Lee, Adv. Mater., 2013, 25, 2746-2752.

75 M. Wang, M. Kim, D. Odkhuu, N. Park, J. Lee, W.-J. Jang, S.-J. Kahng, R. S. Ruoff, Y. J. Song and S. Lee, ACS Nano, 2014, 8 , 5478-5483.

76 S. M. Kim, A. Hsu, P. T. Araujo, Y.-H. Lee, T. Palacios, M. Dresselhaus, J.-C. Idrobo, K. K. Kim and J. Kong, Nano Lett., 2013, 13, 933-941.

77 S. Roth, F. Matsui, T. Greber and J. Osterwalder, Nano Lett., 2013, 13, 2668-2675.

78 W. Yang, G. Chen, Z. Shi, C.-C. Liu, L. Zhang, G. Xie, M. Cheng, D. Wang, R. Yang, D. Shi, K. Watanabe, T. Taniguchi, Y. Yao, Y. Zhang and G. Zhang, Nat. Mater., 2013, 12, 792-797.

79 S. Tang, H. Wang, Y. Zhang, A. Li, H. Xie, X. Liu, L. Liu, T. Li, F. Huang, X. Xie and M. Jiang, Sci. Rep., 2013, 3, 2666.

80 J. H. Meng, X. W. Zhang, H. L. Wang, X. B. Ren, C. H. Jin, Z. G. Yin, X. Liu and H. Liu, Nanoscale, 2015, 7, 16046-16053.

81 C. Zhang, S. Zhao, C. Jin, A. L. Koh, Y. Zhou, W. Xu, Q. Li, Q. Xiong, H. Peng and Z. Liu, Nat. Commun., 2015, 6, 6519.

82 Y. Yang, Q. Fu, H. Li, M. Wei, J. Xiao, W. Wei and X. Bao, ACS Nano, 2015, 9, 11589-11598.

83 S. Roth, T. Greber and J. Osterwalder, ACS Nano, 2016, 10, 11187-11195.

84 X. Song, T. Gao, Y. Nie, J. Zhuang, J. Sun, D. Ma, J. Shi, Y. Lin, F. Ding, Y. Zhang and Z. Liu, Nano Lett., 2016, 16, 6109-6116.

85 Y. Shi, W. Zhou, A.-Y. Lu, W. Fang, Y.-H. Lee, A. L. Hsu, S. M. Kim, K. K. Kim, H. Y. Yang, L.-J. Li, J.-C. Idrobo and J. Kong, Nano Lett., 2012, 12, 2784-2791.

86 X. Ling, Y.-H. Lee, Y. Lin, W. Fang, L. Yu, M. S. Dresselhaus and J. Kong, Nano Lett., 2014, 14, 464-472.

87 H. Ago, H. Endo, P. Solís-Fernández, R. Takizawa, Y. Ohta, Y. Fujita, K. Yamamoto and M. Tsuji, ACS Appl. Mater. Interfaces, 2015, 7, 5265-5273.

88 R. M. Yunus, H. Endo, M. Tsuji and H. Ago, Phys. Chem. Chem. Phys., 2015, 17, 25210-25215.

89 J. A. Miwa, M. Dendzik, S. S. Grønborg, M. Bianchi, J. V. Lauritsen, P. Hofmann and S. Ulstrup, ACS Nano, 2015, 9, 6502-6510.

90 A. Azizi, S. Eichfeld, G. Geschwind, K. Zhang, B. Jiang, D. Mukherjee, L. Hossain, A. F. Piasecki, B. Kabius, J. A. Robinson and N. Alem, ACS Nano, 2015, 9, 4882-4890.

91 J. Shi, M. Liu, J. Wen, X. Ren, X. Zhou, Q. Ji, D. Ma, Y. Zhang, C. Jin, H. Chen, S. Deng, N. Xu, Z. Liu and Y. Zhang, Adv. Mater., 2015, 27, 7086-7092.

92 W. Wan, X. Li, X. Li, B. Xu, L. Zhan, Z. Zhao, P. Zhang, S. Q. Wu, Z. Zhu, H. Huang, Y. Zhou and W. Cai, RSC Adv., 2015, 6, 323-330. 
93 X. Liu, I. Balla, H. Bergeron, G. P. Campbell, M. J. Bedzyk and M. C. Hersam, ACS Nano, 2016, 10, 1067-1075.

$94 \mathrm{H}$. Ago, S. Fukamachi, H. Endo, P. Solís-Fernández, R. M. Yunus, Y. Uchida, V. Panchal, O. Kazakova and M. Tsuji, ACS Nano, 2016, 10, 3233-3240.

95 S. Ulstrup, A. G. Čabo, J. A. Miwa, J. M. Riley, S. S. Grønborg, J. C. Johannsen, C. Cacho, O. Alexander, R. T. Chapman, E. Springate, M. Bianchi, M. Dendzik, J. V. Lauritsen, P. D. C. King and P. Hofmann, ACS Nano, 2016, 10, 6315-6322.

96 W. Wan, L. Zhan, B. Xu, F. Zhao, Z. Zhu, Y. Zhou, Z. Yang, T. Shih and W. Cai, Small, 2017, n/a-n/a.

97 G. V. Bianco, M. Losurdo, M. M. Giangregorio, A. Sacchetti, P. Prete, N. Lovergine, P. Capezzuto and G. Bruno, RSC Adv., 2015, 5, 98700-98708.

98 A. Rossi, H. Büch, C. D. Rienzo, V. Miseikis, D. Convertino, Ameer Al-Temimy, V. Voliani, M. Gemmi, V. Piazza and C. Coletti, 2D Mater., 2016, 3, 31013.

99 S.-Y. Kim, J. Kwak, J. H. Kim, J.-U. Lee, Y. Jo, S. Y. Kim, H. Cheong, Zonghoon Lee and S.-Y. Kwon, 2D Mater., 2017, 4, 11007.

100 C. Lan, C. Li, S. Wang, T. He, Z. Zhou, D. Wei, H. Guo, H. Yang and Y. Liu, J. Mater. Chem. C, 2017.

101 G. W. Shim, K. Yoo, S.-B. Seo, J. Shin, D. Y. Jung, I.-S. Kang, C. W. Ahn, B. J. Cho and S.-Y. Choi, ACS Nano, 2014, 8, 66556662.

102 Y.-C. Lin, C.-Y. S. Chang, R. K. Ghosh, J. Li, H. Zhu, R. Addou, B. Diaconescu, T. Ohta, X. Peng, N. Lu, M. J. Kim, J. T. Robinson, R. M. Wallace, T. S. Mayer, S. Datta, L.-J. Li and J. A. Robinson, Nano Lett., 2014, 14, 6936-6941.

103 Y.-C. Lin, J. Li, S. C. de la Barrera, S. M. Eichfeld, Y. Nie, R. Addou, P. C. Mende, R. M. Wallace, K. Cho, R. M. Feenstra and J. A. Robinson, Nanoscale, 2016, 8, 8947-8954.

104 M. M. Ugeda, A. J. Bradley, Y. Zhang, S. Onishi, Y. Chen, W. Ruan, C. Ojeda-Aristizabal, H. Ryu, M. T. Edmonds, H.-Z. Tsai, A. Riss, S.-K. Mo, D. Lee, A. Zettl, Z. Hussain, Z.-X. Shen and M. F. Crommie, Nat. Phys., 2016, 12, 92-97.

105 Y.-C. Lin, R. K. Ghosh, R. Addou, N. Lu, S. M. Eichfeld, H. Zhu, M.-Y. Li, X. Peng, M. J. Kim, L.-J. Li, R. M. Wallace, S. Datta and J. A. Robinson, Nat. Commun., 2015, 6, 7311.

106 X. Li, L. Basile, B. Huang, C. Ma, J. Lee, I. V. Vlassiouk, A. A. Puretzky, M.-W. Lin, M. Yoon, M. Chi, J. C. Idrobo, C. M. Rouleau, B. G. Sumpter, D. B. Geohegan and K. Xiao, ACS Nano, 2015, 9, 8078-8088.

107 Z. Ben Aziza, H. Henck, D. Pierucci, M. G. Silly, E. Lhuillier, G. Patriarche, F. Sirotti, M. Eddrief and A. Ouerghi, ACS Nano, 2016, 10, 9679-9686.

108 W. Dang, H. Peng, H. Li, P. Wang and Z. Liu, Nano Lett., 2010, 10, 2870-2876.

109 M. Lin, D. Wu, Y. Zhou, W. Huang, W. Jiang, W. Zheng, S. Zhao, C. Jin, Y. Guo, H. Peng and Z. Liu, J. Am. Chem. Soc., 2013, 135, 13274-13277.

110 K. Vaklinova, A. Hoyer, M. Burghard and K. Kern, Nano Lett., 2016, 16, 2595-2602.

111 H.-K. Hong, J. Jo, D. Hwang, J. Lee, N. Y. Kim, S. Son, J. H. Kim, M.-J. Jin, Y. C. Jun, R. Erni, S. K. Kwak, J.-W. Yoo and Z. Lee, Nano Lett., 2017, 17, 120-127.

112 L. Niu, X. Liu, C. Cong, C. Wu, D. Wu, T. R. Chang, H. Wang, Q. Zeng, J. Zhou, X. Wang, W. Fu, P. Yu, Q. Fu, S. Najmaei, Z. Zhang, B. I. Yakobson, B. K. Tay, W. Zhou, H. T. Jeng, H. Lin, T. C. Sum, C. Jin, H. He, T. Yu and Z. Liu, Adv. Mater., 2015, 27, 7800-7808.

113 C. H. Lui, L. Liu, K. F. Mak, G. W. Flynn and T. F. Heinz, Nature, 2009, 462, 339-341.

114 Y. Zhang, V. W. Brar, C. Girit, A. Zettl and M. F. Crommie, Nat. Phys., 2009, 5, 722-726.

115 J. Jobst, A. J. H. van der Torren, E. E. Krasovskii, J. Balgley, C. R. Dean, R. M. Tromp and S. J. van der Molen, Nat. Commun., 2016, 7, 13621.
116 J. Xue, J. Sanchez-Yamagishi, D. Bulmash, P. Jacquod, A. Deshpande, K. Watanabe, T. Taniguchi, P. Jarillo-Herrero and B. J. LeRoy, Nat. Mater., 2011, 10, 282-285.

117 R. Decker, Y. Wang, V. W. Brar, W. Regan, H.-Z. Tsai, Q. Wu, W. Gannett, A. Zettl and M. F. Crommie, Nano Lett., 2011, 11, 2291-2295.

118 S. M. Kim, A. Hsu, M. H. Park, S. H. Chae, S. J. Yun, J. S. Lee, D.-H. Cho, W. Fang, C. Lee, T. Palacios, M. Dresselhaus, K. K. Kim, Y. H. Lee and J. Kong, Nat. Commun., 2015, 6, 8662.

119 D. H. Tien, J.-Y. Park, K. B. Kim, N. Lee, T. Choi, P. Kim, T. Taniguchi, K. Watanabe and Y. Seo, ACS Appl. Mater. Interfaces, 2016, 8, 3072-3078.

120 Z. Liu, Y. Gong, W. Zhou, L. Ma, J. Yu, J. C. Idrobo, J. Jung, A. H. MacDonald, R. Vajtai, J. Lou and P. M. Ajayan, Nat. Commun., 2013, 4, 2541.

121 R. Zan, Q. M. Ramasse, R. Jalil, T. Georgiou, U. Bangert and K. S. Novoselov, ACS Nano, 2013, 7, 10167-10174.

122 G. Algara-Siller, S. Kurasch, M. Sedighi, O. Lehtinen and U. Kaiser, Appl. Phys. Lett., 2013, 103, 203107.

123 A. M. Goossens, S. C. M. Driessen, T. A. Baart, K. Watanabe, T. Taniguchi and L. M. K. Vandersypen, Nano Lett., 2012, 12, 4656-4660.

124 L. Britnell, R. V. Gorbachev, R. Jalil, B. D. Belle, F. Schedin, M. I. Katsnelson, L. Eaves, S. V. Morozov, A. S. Mayorov, N. M. R. Peres, A. H. Castro Neto, J. Leist, A. K. Geim, L. A. Ponomarenko and K. S. Novoselov, Nano Lett., 2012, 12, 1707-1710.

125 C. R. Dean, A. F. Young, P. Cadden-Zimansky, L. Wang, H. Ren, K. Watanabe, T. Taniguchi, P. Kim, J. Hone and K. L. Shepard, Nat. Phys., 2011, 7, 693-696.

126 A. F. Young, C. R. Dean, L. Wang, H. Ren, P. CaddenZimansky, K. Watanabe, T. Taniguchi, J. Hone, K. L. Shepard and P. Kim, Nat. Phys., 2012, 8, 550-556.

127 F. Amet, A. J. Bestwick, J. R. Williams, L. Balicas, K. Watanabe, T. Taniguchi and D. Goldhaber-Gordon, Nat. Commun., 2015, 6, 5838.

128 P. J. Zomer, M. H. D. Guimarães, N. Tombros and B. J. van Wees, Phys. Rev. B, 2012, 86, 161416.

129 A. F. Young, J. D. Sanchez-Yamagishi, B. Hunt, S. H. Choi, K. Watanabe, T. Taniguchi, R. C. Ashoori and P. JarilloHerrero, Nature, 2014, 505, 528-532.

130 M. Yankowitz, J. Xue, D. Cormode, J. D. SanchezYamagishi, K. Watanabe, T. Taniguchi, P. Jarillo-Herrero, P. Jacquod and B. J. LeRoy, Nat. Phys., 2012, 8, 382-386.

131 C.-H. Park, L. Yang, Y.-W. Son, M. L. Cohen and S. G. Louie, Phys. Rev. Lett., 2008, 101, 126804.

132 B. Hunt, J. D. Sanchez-Yamagishi, A. F. Young, M. Yankowitz, B. J. LeRoy, K. Watanabe, T. Taniguchi, P. Moon, M. Koshino, P. Jarillo-Herrero and R. C. Ashoori, Science, 2013, 340, 1427-1430.

133 E. Wang, X. Lu, S. Ding, W. Yao, M. Yan, G. Wan, K. Deng, S. Wang, G. Chen, L. Ma, J. Jung, A. V. Fedorov, Y. Zhang, G. Zhang and S. Zhou, Nat. Phys., 2016, 12, 1111-1115.

134 C. R. Woods, L. Britnell, A. Eckmann, R. S. Ma, J. C. Lu, H. M. Guo, X. Lin, G. L. Yu, Y. Cao, R. V. Gorbachev, A. V. Kretinin, J. Park, L. A. Ponomarenko, M. I. Katsnelson, Y. N. Gornostyrev, K. Watanabe, T. Taniguchi, C. Casiraghi, H.-J. Gao, A. K. Geim and K. S. Novoselov, Nat. Phys., 2014, 10, 451-456.

135 E. Wang, G. Chen, G. Wan, X. Lu, C. Chen, J. Avila, A. V. Fedorov, Guangyu Zhang, M. C. Asensio, Y. Zhang and S. Zhou, J. Phys. Condens. Matter, 2016, 28, 444002.

136 L. A. Ponomarenko, R. V. Gorbachev, G. L. Yu, D. C. Elias, R. Jalil, A. A. Patel, A. Mishchenko, A. S. Mayorov, C. R. Woods, J. R. Wallbank, M. Mucha-Kruczynski, B. A. Piot, M. Potemski, I. V. Grigorieva, K. S. Novoselov, F. Guinea, V. I. Fal'ko and A. K. Geim, Nature, 2013, 497, 594-597. 
137 C. Handschin, P. Makk, P. Rickhaus, M.-H. Liu, K. Watanabe, T. Taniguchi, K. Richter and C. Schönenberger, Nano Lett., 2017, 17, 328-333.

138 D. Pierucci, H. Henck, J. Avila, A. Balan, C. H. Naylor, G. Patriarche, Y. J. Dappe, M. G. Silly, F. Sirotti, A. T. C. Johnson, M. C. Asensio and A. Ouerghi, Nano Lett., 2016, 16, 40544061.

139 K. M. McCreary, A. T. Hanbicki, J. T. Robinson, E. Cobas, J. C. Culbertson, A. L. Friedman, G. G. Jernigan and B. T. Jonker, Adv. Funct. Mater., 2014, 24, 6449-6454.

140 X. Liu, I. Balla, H. Bergeron and M. C. Hersam, J. Phys. Chem. C, 2016, 120, 20798-20805.

141 H. Coy Diaz, J. Avila, C. Chen, R. Addou, M. C. Asensio and M. Batzill, Nano Lett., 2015, 15, 1135-1140.

142 J. Y. Tan, A. Avsar, J. Balakrishnan, G. K. W. Koon, T. Taychatanapat, E. C. T. O'Farrell, K. Watanabe, T. Taniguchi, G. Eda, A. H. C. Neto and B. Özyilmaz, Appl. Phys. Lett., 2014, 104, 183504.

143 M. Yankowitz, S. Larentis, K. Kim, J. Xue, D. McKenzie, S. Huang, M. Paggen, M. N. Ali, R. J. Cava, E. Tutuc and B. J. LeRoy, Nano Lett., 2015, 15, 1925-1929.

144 H. Tan, Y. Fan, Y. Rong, B. Porter, C. S. Lau, Y. Zhou, Z. He, S. Wang, H. Bhaskaran and J. H. Warner, ACS Appl. Mater. Interfaces, 2016, 8, 1644-1652.

145 C. E. Giusca, I. Rungger, V. Panchal, C. Melios, Z. Lin, Y.-C. Lin, E. Kahn, A. L. Elías, J. A. Robinson, M. Terrones and O. Kazakova, ACS Nano, 2016, 10, 7840-7846.

146 H. Xu, J. Wu, Q. Feng, N. Mao, C. Wang and J. Zhang, Small, 2014, 10, 2300-2306.

147 C.-H. Lee, G.-H. Lee, A. M. van der Zande, W. Chen, Y. Li, M. Han, X. Cui, G. Arefe, C. Nuckolls, T. F. Heinz, J. Guo, J. Hone and P. Kim, Nat. Nanotechnol., 2014, 9, 676-681.

148 M. Massicotte, P. Schmidt, F. Vialla, K. G. Schädler, A. Reserbat-Plantey, K. Watanabe, T. Taniguchi, K. J. Tielrooij and F. H. L. Koppens, Nat. Nanotechnol., 2016, 11, 42-46.

149 H. Tan, Y. Fan, Y. Zhou, Q. Chen, W. Xu and J. H. Warner, ACS Nano, 2016, 10, 7866-7873.

150 Q. A. Vu, J. H. Lee, V. L. Nguyen, Y. S. Shin, S. C. Lim, K. Lee, J. Heo, S. Park, K. Kim, Y. H. Lee and W. J. Yu, Nano Lett., 2017, 17, 453-459.

151 R.-J. Shiue, Y. Gao, Y. Wang, C. Peng, A. D. Robertson, D. K. Efetov, S. Assefa, F. H. L. Koppens, J. Hone and D. Englund, Nano Lett., 2015, 15, 7288-7293.

152 M. Bernardi, M. Palummo and J. C. Grossman, Nano Lett., 2013, 13, 3664-3670.

153 M. Shanmugam, R. Jacobs-Gedrim, E. S. Song and B. Yu, Nanoscale, 2014, 6, 12682-12689.

154 Y. Tsuboi, F. Wang, D. Kozawa, K. Funahashi, S. Mouri, Y. Miyauchi, T. Takenobu and K. Matsuda, Nanoscale, 2015, 7, 14476-14482.

155 F. Withers, O. Del Pozo-Zamudio, A. Mishchenko, A. P. Rooney, A. Gholinia, K. Watanabe, T. Taniguchi, S. J. Haigh, A. K. Geim, A. I. Tartakovskii and K. S. Novoselov, Nat. Mater., 2015, 14, 301-306.

156 F. Withers, O. Del Pozo-Zamudio, S. Schwarz, S. Dufferwiel, P. M. Walker, T. Godde, A. P. Rooney, A. Gholinia, C. R. Woods, P. Blake, S. J. Haigh, K. Watanabe, T. Taniguchi, I. L. Aleiner, A. K. Geim, V. I. Fal'ko, A. I. Tartakovskii and K. S. Novoselov, Nano Lett., 2015, 15, 8223-8228.

157 C. Palacios-Berraquero, M. Barbone, D. M. Kara, X. Chen, I. Goykhman, D. Yoon, A. K. Ott, J. Beitner, K. Watanabe, T. Taniguchi, A. C. Ferrari and M. Atatüre, Nat. Commun., 2016, 7, 12978.

158 G. Clark, J. R. Schaibley, J. Ross, T. Taniguchi, K. Watanabe, J. R. Hendrickson, S. Mou, W. Yao and X. Xu, Nano Lett., 2016, 16, 3944-3948.

159 S. Schwarz, A. Kozikov, F. Withers, J. K. Maguire, A. P. Foster, S. Dufferwiel, L. Hague, M. N. Makhonin, L. R. Wilson,
A. K. Geim, K. S. Novoselov and A. I. Tartakovskii, 2D Mater., 2016, 3, 25038.

160 Y. Gao, R.-J. Shiue, X. Gan, L. Li, C. Peng, I. Meric, L. Wang, A. Szep, D. Walker, J. Hone and D. Englund, Nano Lett., 2015, 15, 2001-2005.

161 C.-H. Liu, G. Clark, T. Fryett, S. Wu, J. Zheng, F. Hatami, X. Xu and A. Majumdar, Nano Lett., 2017, 17, 200-205.

162 Q. A. Vu, Y. S. Shin, Y. R. Kim, V. L. Nguyen, W. T. Kang, H. Kim, D. H. Luong, I. M. Lee, K. Lee, D.-S. Ko, J. Heo, S. Park, Y. H. Lee and W. J. Yu, Nat. Commun., 2016, 7, 12725.

163 L. Ju, J. Velasco Jr, E. Huang, S. Kahn, C. Nosiglia, H.-Z. Tsai, W. Yang, T. Taniguchi, K. Watanabe, Y. Zhang, G. Zhang, M. Crommie, A. Zettl and F. Wang, Nat. Nanotechnol., 2014, 9, 348-352.

164 J. Velasco, L. Ju, D. Wong, S. Kahn, J. Lee, H.-Z. Tsai, C. Germany, S. Wickenburg, J. Lu, T. Taniguchi, K. Watanabe, A. Zettl, F. Wang and M. F. Crommie, Nano Lett., 2016, 16, 1620-1625.

165 B. Cho, J. Yoon, S. K. Lim, A. R. Kim, D.-H. Kim, S.-G. Park, J.-D. Kwon, Y.-J. Lee, K.-H. Lee, B. H. Lee, H. C. Ko and M. G. Hahm, ACS Appl. Mater. Interfaces, 2015, 7, 16775-16780.

166 L. Yu, Y.-H. Lee, X. Ling, E. J. G. Santos, Y. C. Shin, Y. Lin, M. Dubey, E. Kaxiras, J. Kong, H. Wang and T. Palacios, Nano Lett., 2014, 14, 3055-3063.

167 T. Roy, M. Tosun, J. S. Kang, A. B. Sachid, S. B. Desai, M. Hettick, C. C. Hu and A. Javey, ACS Nano, 2014, 8, 62596264.

168 H.-J. Chuang, X. Tan, N. J. Ghimire, M. M. Perera, B. Chamlagain, M. M.-C. Cheng, J. Yan, D. Mandrus, D. Tománek and Z. Zhou, Nano Lett., 2014, 14, 3594-3601.

169 X. Cui, G.-H. Lee, Y. D. Kim, G. Arefe, P. Y. Huang, C.-H. Lee, D. A. Chenet, X. Zhang, L. Wang, F. Ye, F. Pizzocchero, B. S. Jessen, K. Watanabe, T. Taniguchi, D. A. Muller, T. Low, P. Kim and J. Hone, Nat. Nanotechnol., 2015, 10, 534-540.

170 C.-J. Shih, Q. H. Wang, Y. Son, Z. Jin, D. Blankschtein and M. S. Strano, ACS Nano, 2014, 8, 5790-5798.

171 L. Britnell, R. V. Gorbachev, A. K. Geim, L. A. Ponomarenko, A. Mishchenko, M. T. Greenaway, T. M. Fromhold, K. S. Novoselov and L. Eaves, Nat. Commun., 2013, 4, 1794.

172 A. Mishchenko, J. S. Tu, Y. Cao, R. V. Gorbachev, J. R. Wallbank, M. T. Greenaway, V. E. Morozov, S. V. Morozov, M. J. Zhu, S. L. Wong, F. Withers, C. R. Woods, Y.-J. Kim, K. Watanabe, T. Taniguchi, E. E. Vdovin, O. Makarovsky, T. M. Fromhold, V. I. Fal'ko, A. K. Geim, L. Eaves and K. S. Novoselov, Nat. Nanotechnol., 2014, 9, 808-813.

173 S. Kang, B. Fallahazad, K. Lee, H. Movva, K. Kim, C. M. Corbet, T. Taniguchi, K. Watanabe, L. Colombo, L. F. Register, E. Tutuc and S. K. Banerjee, IEEE Electron Device Lett., 2015, 36, 405-407.

174 L. Brey, Phys. Rev. Appl., 2014, 2, 14003.

175 H. Tian, Z. Tan, C. Wu, X. Wang, M. A. Mohammad, D. Xie, Y. Yang, J. Wang, L.-J. Li, J. Xu and T.-L. Ren, Sci. Rep., 2014, 4.

176 J. Shim, H. S. Kim, Y. S. Shim, D.-H. Kang, H.-Y. Park, J. Lee, J. Jeon, S. J. Jung, Y. J. Song, W.-S. Jung, J. Lee, S. Park, J. Kim, S. Lee, Y.-H. Kim and J.-H. Park, Adv. Mater., 2016, 28, 52935299.

177 Y. Sata, R. Moriya, S. Morikawa, N. Yabuki, S. Masubuchi and T. Machida, Appl. Phys. Lett., 2015, 107, 23109.

178 Y. Choi, J. Kang, D. Jariwala, M. S. Kang, T. J. Marks, M. C. Hersam and J. H. Cho, Adv. Mater., 2016, 28, 3742-3748.

179 D. Akinwande, N. Petrone and J. Hone, Nat. Commun., 2014, 5, 5678.

180 H. Jang, Y. J. Park, X. Chen, T. Das, M.-S. Kim and J.-H. Ahn, Adv. Mater., 2016, 28, 4184-4202.

181 A. Avsar, J. Y. Tan, T. Taychatanapat, J. Balakrishnan, G. K. W. Koon, Y. Yeo, J. Lahiri, A. Carvalho, A. S. Rodin, E. C. T. 
O'Farrell, G. Eda, A. H. C. Neto and B. Özyilmaz, Nat. Commun., 2014, 5, 4875.

182 Z. Wang, D.-K. Ki, H. Chen, H. Berger, A. H. MacDonald and A. F. Morpurgo, Nat. Commun., 2015, 6, 8339.

183 Z. Wang, D.-K. Ki, J. Y. Khoo, D. Mauro, H. Berger, L. S. Levitov and A. F. Morpurgo, Phys. Rev. X, 2016, 6, 41020.

184 R. Lu, J. Liu, H. Luo, V. Chikan and J. Z. Wu, Sci. Rep., 2016, 6, 19161.

185 L. Zhang, Y. Yan, H.-C. Wu, D. Yu and Z.-M. Liao, ACS Nano, 2016, 10, 3816-3822.

186 J. W. Colson, A. R. Woll, A. Mukherjee, M. P. Levendorf, E. L. Spitler, V. B. Shields, M. G. Spencer, J. Park and W. R. Dichtel, Science, 2011, 332, 228-231.

187 L. Xu, X. Zhou, W. Q. Tian, T. Gao, Y. F. Zhang, S. Lei and Z. F. Liu, Angew. Chem. Int. Ed., 2014, 53, 9564-9568.

188 M. Woszczyna, A. Winter, M. Grothe, A. Willunat, S. Wundrack, R. Stosch, T. Weimann, F. Ahlers and A. Turchanin, Adv. Mater., 2014, 26, 4831-4837.

189 C. Ojeda-Aristizabal, W. Bao and M. S. Fuhrer, Phys. Rev. $B, 2013,88,35435$.

190 G. Oh, J.-S. Kim, J. H. Jeon, E. Won, J. W. Son, D. H. Lee, C. K. Kim, J. Jang, T. Lee and B. H. Park, ACS Nano, 2015, 9, 7515-7522.

191 B. H. Seo, J. Youn and M. Shim, ACS Nano, 2014, 8, 88318836.

192 Q. H. Wang and M. C. Hersam, Nat. Chem., 2009, 1, 206211.

193 B. Li, A. V. Klekachev, M. Cantoro, C. Huyghebaert, A. Stesmans, I. Asselberghs, S. D. Gendt and S. D. Feyter, Nanoscale, 2013, 5, 9640-9644.

194 Y. Ogawa, T. Niu, S. L. Wong, M. Tsuji, A. T. S. Wee, W. Chen and H. Ago, J. Phys. Chem. C, 2013, 117, 21849-21855.

195 B. Wu, Y. Zhao, H. Nan, Z. Yang, Y. Zhang, H. Zhao, D. He, Z. Jiang, X. Liu, Y. Li, Y. Shi, Z. Ni, J. Wang, J.-B. Xu and X. Wang, Nano Lett., 2016, 16, 3754-3759.

196 H.-C. Cheng, G. Wang, D. Li, Q. He, A. Yin, Y. Liu, H. Wu, M. Ding, Y. Huang and X. Duan, Nano Lett., 2016, 16, 367373.

197 L. Ci, L. Song, C. Jin, D. Jariwala, D. Wu, Y. Li, A. Srivastava, Z. F. Wang, K. Storr, L. Balicas, F. Liu and P. M. Ajayan, Nat. Mater., 2010, 9, 430-435.

198 J. Jung, Z. Qiao, Q. Niu and A. H. MacDonald, Nano Lett., 2012, 12, 2936-2940.

199 Q. Sun, Y. Dai, Y. Ma, W. Wei and B. Huang, RSC Adv., 2015, 5, 33037-33043.

200 X. Ling, Y. Lin, Q. Ma, Z. Wang, Y. Song, L. Yu, S. Huang, W. Fang, X. Zhang, A. L. Hsu, Y. Bie, Y.-H. Lee, Y. Zhu, L. Wu, J. Li, P. Jarillo-Herrero, M. Dresselhaus, T. Palacios and J. Kong, Adv. Mater., 2016, 28, 2322-2329.

201 M. Zhao, Y. Ye, Y. Han, Y. Xia, H. Zhu, S. Wang, Y. Wang, D. A. Muller and X. Zhang, Nat. Nanotechnol., 2016, 11, 954959.

202 M. H. D. Guimarães, H. Gao, Y. Han, K. Kang, S. Xie, C.-J. Kim, D. A. Muller, D. C. Ralph and J. Park, ACS Nano, 2016, 10, 6392-6399.

203 X. Chen, Y. Park, T. Das, H. Jang, J.-B. Lee and J.-H. Ahn, Nanoscale, 2016, 8, 15181-15188.

204 X. Lin, P. Liu, Y. Wei, Q. Li, J. Wang, Y. Wu, C. Feng, L. Zhang, S. Fan and K. Jiang, Nat. Commun., 2013, 4, 2920.

205 Z. Yan, Z. Peng, G. Casillas, J. Lin, C. Xiang, H. Zhou, Y. Yang, G. Ruan, A.-R. O. Raji, E. L. G. Samuel, R. H. Hauge, M. J. Yacaman and J. M. Tour, ACS Nano, 2014, 8, 5061-5068.

206 X. Li, J. Sha, S.-K. Lee, Y. Li, Y. Ji, Y. Zhao and J. M. Tour, ACS Nano, 2016, 10, 7307-7313.

207 Y. Li, Z. Peng, E. Larios, G. Wang, J. Lin, Z. Yan, F. RuizZepeda, M. José-Yacamán and J. M. Tour, ACS Nano, 2015, 9, 532-538.
208 Y. Liu, F. Wang, X. Wang, X. Wang, E. Flahaut, X. Liu, Y. Li, X. Wang, Y. Xu, Y. Shi and R. Zhang, Nat. Commun., 2015, 6, 8589.

209 S. H. Chae, W. J. Yu, J. J. Bae, D. L. Duong, D. Perello, H. Y. Jeong, Q. H. Ta, T. H. Ly, Q. A. Vu, M. Yun, X. Duan and Y. H. Lee, Nat. Mater., 2013, 12, 403-409.

210 G. Konstantatos, M. Badioli, L. Gaudreau, J. Osmond, M. Bernechea, F. P. G. de Arquer, F. Gatti and F. H. L. Koppens, Nat. Nanotechnol., 2012, 7, 363-368.

211 I. Nikitskiy, S. Goossens, D. Kufer, T. Lasanta, G. Navickaite, F. H. L. Koppens and G. Konstantatos, Nat. Commun., 2016, 7, 11954.

212 Z. Sun, Z. Liu, J. Li, G. Tai, S.-P. Lau and F. Yan, Adv. Mater., 2012, 24, 5878-5883.

213 L. A. Chernozatonskii, A. G. Kvashnin and P. B. Sorokin, Nanotechnology, 2016, 27, 365201.

214 Z. Zheng, X. Zhang, C. Neumann, D. Emmrich, A. Winter, H. Vieker, W. Liu, M. Lensen, A. Gölzhäuser and A. Turchanin, Nanoscale, 2015, 7, 13393-13397.

215 J. Wan, S. D. Lacey, J. Dai, W. Bao, M. S. Fuhrer and L. Hu, Chem. Soc. Rev., 2016, 45, 6742-6765.

216 K. S. Vasu, E. Prestat, J. Abraham, J. Dix, R. J. Kashtiban, J. Beheshtian, J. Sloan, P. Carbone, M. Neek-Amal, S. J. Haigh, A. K. Geim and R. R. Nair, Nat. Commun., 2016, 7, 12168.

217 N. Kim, K. S. Kim, N. Jung, L. Brus and P. Kim, Nano Lett., 2011, 11, 860-865.

218 T. H. Bointon, I. Khrapach, R. Yakimova, A. V. Shytov, M. F. Craciun and S. Russo, Nano Lett., 2014, 14, 1751-1755.

219 K. Kanetani, K. Sugawara, T. Sato, R. Shimizu, K. Iwaya, T. Hitosugi and T. Takahashi, Proc. Natl. Acad. Sci., 2012, 109, 19610-19613.

220 S. Ichinokura, K. Sugawara, A. Takayama, T. Takahashi and S. Hasegawa, ACS Nano, 2016, 10, 2761-2765.

221 D. Zhan, L. Sun, Z. H. Ni, L. Liu, X. F. Fan, Y. Wang, T. Yu, Y. M. Lam, W. Huang and Z. X. Shen, Adv. Funct. Mater., 2010, 20, 3504-3509.

222 W. Zhao, P. H. Tan, J. Liu and A. C. Ferrari, J. Am. Chem. Soc., 2011, 133, 5941-5946.

223 I. Khrapach, F. Withers, T. H. Bointon, D. K. Polyushkin, W. L. Barnes, S. Russo and M. F. Craciun, Adv. Mater., 2012, 24, 2844-2849.

224 D. J. Wehenkel, T. H. Bointon, T. Booth, P. Bøggild, M. F. Craciun and S. Russo, Sci. Rep., 2015, 5, 7609.

225 D. Zhan, J. X. Yan, Z. H. Ni, L. Sun, L. F. Lai, L. Liu, X. Y. Liu and Z. X. Shen, Small, 2015, 11, 1177-1182.

226 W. Bao, J. Wan, X. Han, X. Cai, H. Zhu, D. Kim, D. Ma, Y. Xu, J. N. Munday, H. D. Drew, M. S. Fuhrer and L. Hu, Nat. Commun., 2014, 5, 4224

227 R. J. Smith, P. J. King, M. Lotya, C. Wirtz, U. Khan, S. De, A. O'Neill, G. S. Duesberg, J. C. Grunlan, G. Moriarty, J. Chen, J. Wang, A. I. Minett, V. Nicolosi and J. N. Coleman, Adv. Mater., 2011, 23, 3944-3948.

228 J. Zheng, H. Zhang, S. Dong, Y. Liu, C. Tai Nai, H. Suk Shin, H. Young Jeong, B. Liu and K. Ping Loh, Nat. Commun., 2014, 5, 2995.

229 Y. Jung, Y. Zhou and J. J. Cha, Inorg. Chem. Front., 2016, 3, 452-463.

230 Z. Y. Xia, S. Pezzini, E. Treossi, G. Giambastiani, F. Corticelli, V. Morandi, A. Zanelli, V. Bellani and V. Palermo, Adv. Funct. Mater., 2013, 23, 4684-4693.

231 A. M. Abdelkader, A. J. Cooper, R. A. W. Dryfe and I. A. Kinloch, Nanoscale, 2015, 7, 6944-6956.

232 A. Ambrosi and M. Pumera, Chem. - Eur. J., 2016, 22, 153-159.

233 C. Backes, T. M. Higgins, A. Kelly, C. Boland, A. Harvey, D. Hanlon and J. N. Coleman, Chem. Mater., 2017, 29, 243-255.

234 D. R. Dreyer, S. Park, C. W. Bielawski and R. S. Ruoff, Chem. Soc. Rev., 2010, 39, 228-240. 
235 Y. Hernandez, V. Nicolosi, M. Lotya, F. M. Blighe, Z. Sun, S. De, I. T. McGovern, B. Holland, M. Byrne, Y. K. Gun'Ko, J. J. Boland, P. Niraj, G. Duesberg, S. Krishnamurthy, R. Goodhue, J. Hutchison, V. Scardaci, A. C. Ferrari and J. N. Coleman, Nat. Nanotechnol., 2008, 3, 563-568.

236 J. N. Coleman, M. Lotya, A. O'Neill, S. D. Bergin, P. J. King, U. Khan, K. Young, A. Gaucher, S. De, R. J. Smith, I. V. Shvets, S. K. Arora, G. Stanton, H.-Y. Kim, K. Lee, G. T. Kim, G. S. Duesberg, T. Hallam, J. J. Boland, J. J. Wang, J. F. Donegan, J. C. Grunlan, G. Moriarty, A. Shmeliov, R. J. Nicholls, J. M. Perkins, E. M. Grieveson, K. Theuwissen, D. W. McComb, P. D. Nellist and V. Nicolosi, Science, 2011, 331, 568-571.

237 L. Niu, J. N. Coleman, H. Zhang, H. Shin, M. Chhowalla and Z. Zheng, Small, 2016, 12, 272-293.

238 Q. H. Wang, K. Kalantar-Zadeh, A. Kis, J. N. Coleman and M. S. Strano, Nat. Nanotechnol., 2012, 7, 699-712.

239 M. Chhowalla, H. S. Shin, G. Eda, L.-J. Li, K. P. Loh and H. Zhang, Nat. Chem., 2013, 5, 263-275.

240 C. Tan and H. Zhang, Chem. Soc. Rev., 2015, 44, 27132731.

241 Y. Hu, X. Li, A. Lushington, M. Cai, D. Geng, M. N. Banis, R. Li and X. Sun, ECS J. Solid State Sci. Technol., 2013, 2, M3034-M3039.

242 K.-J. Huang, L. Wang, Y.-J. Liu, Y.-M. Liu, H.-B. Wang, T. Gan and L.-L. Wang, Int. J. Hydrog. Energy, 2013, 38, 1402714034.

243 L. Peng, X. Peng, B. Liu, C. Wu, Y. Xie and G. Yu, Nano Lett., 2013, 13, 2151-2157.

244 L. David, R. Bhandavat and G. Singh, ACS Nano, 2014, 8, 1759-1770.

245 M. A. Bissett, I. A. Kinloch and R. A. W. Dryfe, ACS Appl. Mater. Interfaces, 2015, 7, 17388-17398.

246 X. Feng, Z. Yan, N. Chen, Y. Zhang, Y. Ma, X. Liu, Q. Fan, L. Wang and W. Huang, J. Mater. Chem. A, 2013, 1, 1281812825.

247 G. Huang, T. Chen, W. Chen, Z. Wang, K. Chang, L. Ma, F. Huang, D. Chen and J. Y. Lee, Small, 2013, 9, 3693-3703.

248 S. Ratha and C. S. Rout, ACS Appl. Mater. Interfaces, 2013, 5, 11427-11433.

249 A. Ramadoss, T. Kim, G.-S. Kim and S. J. Kim, New J. Chem., 2014, 38, 2379-2385.

250 L. Jiang, B. Lin, X. Li, X. Song, H. Xia, L. Li and H. Zeng, ACS Appl. Mater. Interfaces, 2016, 8, 2680-2687.

251 D. R. Rolison, J. W. Long, J. C. Lytle, A. E. Fischer, C. P. Rhodes, T. M. McEvoy, M. E. Bourg and A. M. Lubers, Chem. Soc. Rev., 2009, 38, 226-252.

252 M. Pumera, Energy Environ. Sci., 2011, 4, 668-674.

253 D. Chen, L. Tang and J. Li, Chem. Soc. Rev., 2010, 39, 3157-3180.

254 R. Raccichini, A. Varzi, S. Passerini and B. Scrosati, Nat. Mater., 2015, 14, 271-279.

255 C. N. R. Rao, K. Gopalakrishnan and U. Maitra, ACS Appl. Mater. Interfaces, 2015, 7, 7809-7832.

256 M. A. Bissett, S. D. Worrall, I. A. Kinloch and R. A. W. Dryfe, Electrochimica Acta, 2016, 201, 30-37.

257 M. Velický, M. A. Bissett, P. S. Toth, H. V. Patten, S. D. Worrall, A. N. J. Rodgers, E. W. Hill, I. A. Kinloch, K. S. Novoselov, T. Georgiou, L. Britnell and R. A. W. Dryfe, Phys. Chem. Chem. Phys., 2015, 17, 17844-17853.

258 M. Velický, M. A. Bissett, C. R. Woods, P. S. Toth, T. Georgiou, I. A. Kinloch, K. S. Novoselov and R. A. W. Dryfe, Nano Lett., 2016, 16, 2023-2032.

259 B. Zhang, X. Ji, K. Xu, C. Chen, X. Xiong, J. Xiong, Y. Yao, L. Miao and J. Jiang, Electrochimica Acta, 2016, 217, 1-8.

260 S. Chen, J. Zhu, X. Wu, Q. Han and X. Wang, ACS Nano, 2010, 4, 2822-2830.

261 Z. Fan, J. Yan, T. Wei, L. Zhi, G. Ning, T. Li and F. Wei, Adv. Funct. Mater., 2011, 21, 2366-2375.
262 M. Zhi, C. Xiang, J. Li, M. Li and N. Wu, Nanoscale, 2013, 5, 72-88.

263 K. Chang and W. Chen, ACS Nano, 2011, 5, 4720-4728.

264 Z. Wang, T. Chen, W. Chen, K. Chang, L. Ma, G. Huang, D. Chen and J. Y. Lee, J. Mater. Chem. A, 2013, 1, 2202-2210.

265 E. G. da Silveira Firmiano, A. C. Rabelo, C. J. Dalmaschio, A. N. Pinheiro, E. C. Pereira, W. H. Schreiner and E. R. Leite, Adv. Energy Mater., 2014, 4, 1301380.

266 H. Li, K. Yu, H. Fu, B. Guo, X. Lei and Z. Zhu, J. Phys. Chem. C, 2015, 119, 7959-7968.

267 X. Xie, Z. Ao, D. Su, J. Zhang and G. Wang, Adv. Funct. Mater., 2015, 25, 1393-1403.

268 A. Gigot, M. Fontana, M. Serrapede, M. Castellino, S. Bianco, M. Armandi, B. Bonelli, C. F. Pirri, E. Tresso and P. Rivolo, ACS Appl. Mater. Interfaces, 2016, 8, 32842-32852.

269 W. S. V. Lee, E. Peng, T. A. J. Loh, X. Huang and J. M. Xue, Nanoscale, 2016, 8, 8042-8047.

270 T. Sun, Z. Li, X. Liu, L. Ma, J. Wang and S. Yang, J. Power Sources, 2016, 331, 180-188.

271 Y. Teng, H. Zhao, Z. Zhang, Z. Li, Q. Xia, Y. Zhang, L. Zhao, X. Du, Z. Du, P. Lv and K. Świerczek, ACS Nano, 2016, 10, 8526-8535.

272 R. Thangappan, S. Kalaiselvam, A. Elayaperumal, R. Jayavel, M. Arivanandhan, R. Karthikeyan and Y. Hayakawa, Dalton Trans., 2016, 45, 2637-2646.

273 N. Savjani, E. A. Lewis, M. A. Bissett, J. R. Brent, R. A. W. Dryfe, S. J. Haigh and P. O'Brien, Chem. Mater., 2016, 28, 657-664.

274 P. Simon and Y. Gogotsi, Nat. Mater., 2008, 7, 845-854.

275 J. Chen, C. Li and G. Shi, J. Phys. Chem. Lett., 2013, 4, 1244-1253.

276 G. Xiong, C. Meng, R. G. Reifenberger, P. P. Irazoqui and T. S. Fisher, Electroanalysis, 2014, 26, 30-51.

277 M. D. Stoller and R. S. Ruoff, Energy Environ. Sci., 2010, 3, 1294-1301.

278 Q. Cheng, J. Tang, J. Ma, H. Zhang, N. Shinya and L.-C. Qin, Carbon, 2011, 49, 2917-2925.

279 M. Beidaghi and C. Wang, Adv. Funct. Mater., 2012, 22, 4501-4510.

280 M. A. Worsley, S. J. Shin, M. D. Merrill, J. Lenhardt, A. J. Nelson, L. Y. Woo, A. E. Gash, T. F. Baumann and C. A. Orme, ACS Nano, 2015, 9, 4698-4705.

281 Y. Lin, F. Liu, G. Casano, R. Bhavsar, I. A. Kinloch and B. Derby, Adv. Mater., 2016, 28, 7993-8000.

282 J. Biener, M. Stadermann, M. Suss, M. A. Worsley, M. M. Biener, K. A. Rose and T. F. Baumann, Energy Environ. Sci., 2011, 4, 656-667.

283 M. A. Py and R. R. Haering, Can. J. Phys., 1983, 61, 76-84. 284 G. Eda, H. Yamaguchi, D. Voiry, T. Fujita, M. Chen and M. Chhowalla, Nano Lett., 2011, 11, 5111-5116.

285 M. Acerce, D. Voiry and M. Chhowalla, Nat. Nanotechnol., 2015, 10, 313-318.

286 Q. Mahmood, S. K. Park, K. D. Kwon, S.-J. Chang, J.-Y. Hong, G. Shen, Y. M. Jung, T. J. Park, S. W. Khang, W. S. Kim, J. Kong and H. S. Park, Adv. Energy Mater., 2016, 6, 1501115.

287 Y.-C. Lin, D. O. Dumcenco, Y.-S. Huang and K. Suenaga, Nat. Nanotechnol., 2014, 9, 391-396.

288 J. M. Tarascon and M. Armand, Nature, 2001, 414, 359367.

289 G. Wang, X. Shen, J. Yao and J. Park, Carbon, 2009, 47, 2049-2053.

290 G. Kucinskis, G. Bajars and J. Kleperis, J. Power Sources, 2013, 240, 66-79.

291 C. Feng, J. Ma, H. Li, R. Zeng, Z. Guo and H. Liu, Mater. Res. Bull., 2009, 44, 1811-1815.

292 X. Rui, H. Tan and Q. Yan, Nanoscale, 2014, 6, 98899924. 
293 X. Hu, W. Zhang, X. Liu, Y. Mei and Y. Huang, Chem. Soc. Rev., 2015, 44, 2376-2404.

294 J. Cabana, L. Monconduit, D. Larcher and M. R. Palacín, Adv. Mater., 2010, 22, E170-E192.

295 T. Stephenson, Z. Li, B. Olsen and D. Mitlin, Energy Environ. Sci., 2014, 7, 209-231.

296 Z. Wang, L. Ma, W. Chen, G. Huang, D. Chen, L. Wang and J. Y. Lee, RSC Adv., 2013, 3, 21675-21684.

297 Y.-X. Wang, S.-L. Chou, D. Wexler, H.-K. Liu and S.-X. Dou, Chem. - Eur. J., 2014, 20, 9607-9612.

298 G. Cunningham, M. Lotya, N. McEvoy, G. S. Duesberg, P. van der Schoot and J. N. Coleman, Nanoscale, 2012, 4, 62606264.

299 X. Wang, X. Lu, B. Liu, D. Chen, Y. Tong and G. Shen, Adv. Mater., 2014, 26, 4763-4782.

300 X. Wang, X. Shen, Z. Wang, R. Yu and L. Chen, ACS Nano, 2014, 8, 11394-11400.

301 C. C. Mayorga-Martinez, A. Ambrosi, A. Y. S. Eng, Z. Sofer and M. Pumera, Electrochem. Commun., 2015, 56, 24-28.

302 G. A. Muller, J. B. Cook, H.-S. Kim, S. H. Tolbert and B. Dunn, Nano Lett., 2015, 15, 1911-1917.

303 S. K. Balasingam, J. S. Lee and Y. Jun, Dalton Trans., 2016, 45, 9646-9653.

304 D. H. Youn, C. Jo, J. Y. Kim, J. Lee and J. S. Lee, J. Power Sources, 2015, 295, 228-234.

305 Z. Luo, J. Zhou, L. Wang, G. Fang, A. Pan and S. Liang, J. Mater. Chem. A, 2016, 4, 15302-15308.

306 J. R. Brent, N. Savjani, E. A. Lewis, S. J. Haigh, D. J. Lewis and P. O'Brien, Chem. Commun., 2014, 50, 13338-13341.

307 J. Kang, J. D. Wood, S. A. Wells, J.-H. Lee, X. Liu, K.-S. Chen and M. C. Hersam, ACS Nano, 2015, 9, 3596-3604.

308 E. A. Lewis, J. R. Brent, B. Derby, S. J. Haigh and D. J. Lewis, Chem. Commun., 2017, 53, 1445-1458.

309 D. Hanlon, C. Backes, E. Doherty, C. S. Cucinotta, N. C. Berner, C. Boland, K. Lee, A. Harvey, P. Lynch, Z. Gholamvand, S. Zhang, K. Wang, G. Moynihan, A. Pokle, Q. M. Ramasse, N. McEvoy, W. J. Blau, J. Wang, G. Abellan, F. Hauke, A. Hirsch, S. Sanvito, D. D. O'Regan, G. S. Duesberg, V. Nicolosi and J. N. Coleman, Nat. Commun., 2015, 6, 8563.

310 Y. Zhang, H. Wang, Z. Luo, H. T. Tan, B. Li, S. Sun, Z. Li, Y. Zong, Z. J. Xu, Y. Yang, K. A. Khor and Q. Yan, Adv. Energy Mater., 2016, 6, 1600453.

311 J. Sun, H.-W. Lee, M. Pasta, H. Yuan, G. Zheng, Y. Sun, Y. Li and Y. Cui, Nat. Nanotechnol., 2015, 10, 980-985.

312 D. Voiry, J. Yang and M. Chhowalla, Adv. Mater., 2016, 28, 6197-6206.

313 T. F. Jaramillo, K. P. Jørgensen, J. Bonde, J. H. Nielsen, S. Horch and I. Chorkendorff, Science, 2007, 317, 100-102.

314 H. Wang, Q. Zhang, H. Yao, Z. Liang, H.-W. Lee, P.-C. Hsu, G. Zheng and Y. Cui, Nano Lett., 2014, 14, 7138-7144.

315 Y.-R. An, X.-L. Fan, Z.-F. Luo and W.-M. Lau, Nano Lett., 2017, 17, 368-376.

316 Y. Li, H. Wang, L. Xie, Y. Liang, G. Hong and H. Dai, J. Am. Chem. Soc., 2011, 133, 7296-7299.

317 P. S. Toth, M. Velický, M. A. Bissett, T. J. A. Slater, N. Savjani, A. K. Rabiu, A. M. Rakowski, J. R. Brent, S. J. Haigh, P. O'Brien and R. A. W. Dryfe, Adv. Mater., 2016, 28, 82568264.

318 F. Reymond, D. Fermín, H. J. Lee and H. H. Girault, Electrochimica Acta, 2000, 45, 2647-2662.

319 F. Reymond and H. H. Girault, in Encyclopedia of Analytical Chemistry, John Wiley \& Sons, Ltd, 2006.

320 S. G. Booth and R. A. W. Dryfe, J. Phys. Chem. C, 2015, 119, 23295-23309.

321 M. A. Bissett, Y. Takesaki, M. Tsuji and H. Ago, RSC Adv., 2014, 4, 52215-52219. 

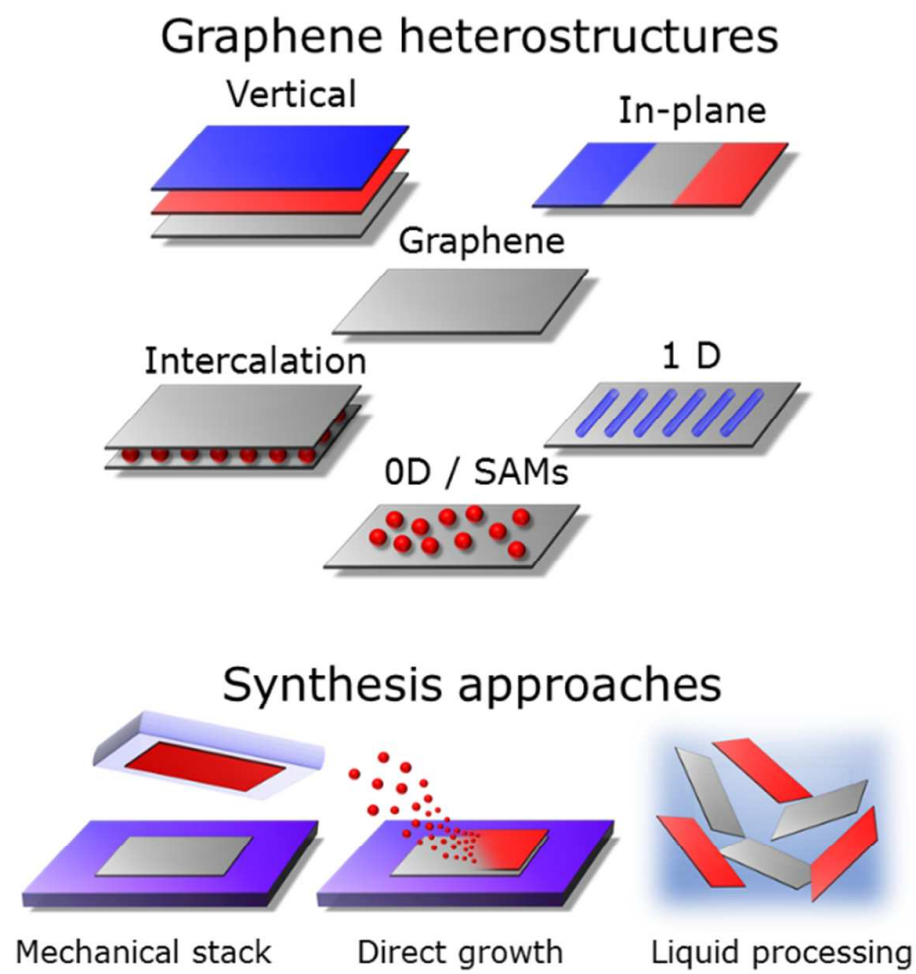

Figure 1. Schematic overview of the different kinds of graphene heterostructures introduced in in this word (top), along with the methods usually employed to fabricate them. 

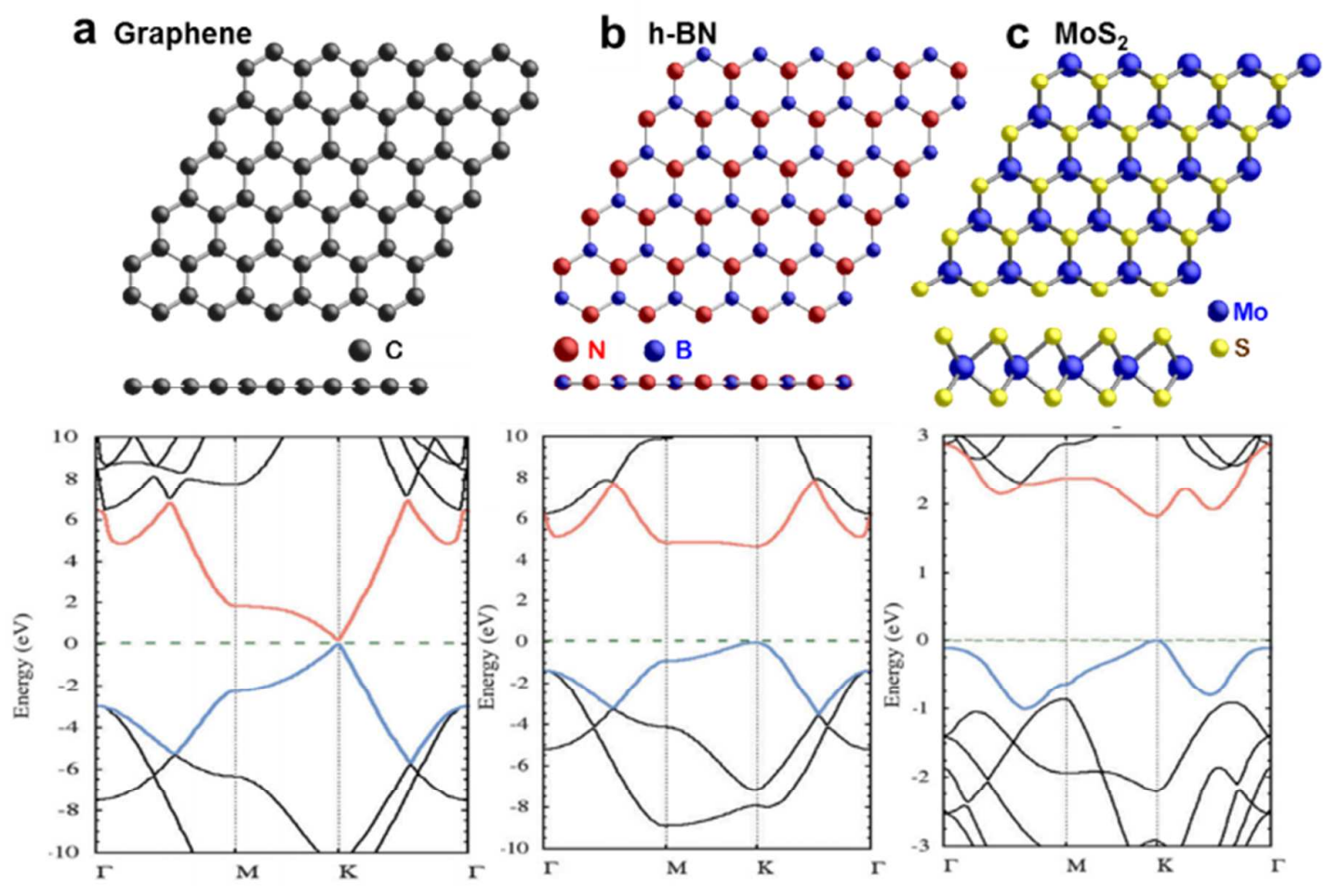

Figure 2. Chemical (top) and band (bottom) structures of some of the most representative 2D materials. (a) Graphene, (b) $h-\mathrm{BN}$, (c) $\mathrm{MoS}_{2}$. Band structures adapted from ref. 10 with permission from The Royal Society of Chemistry. 

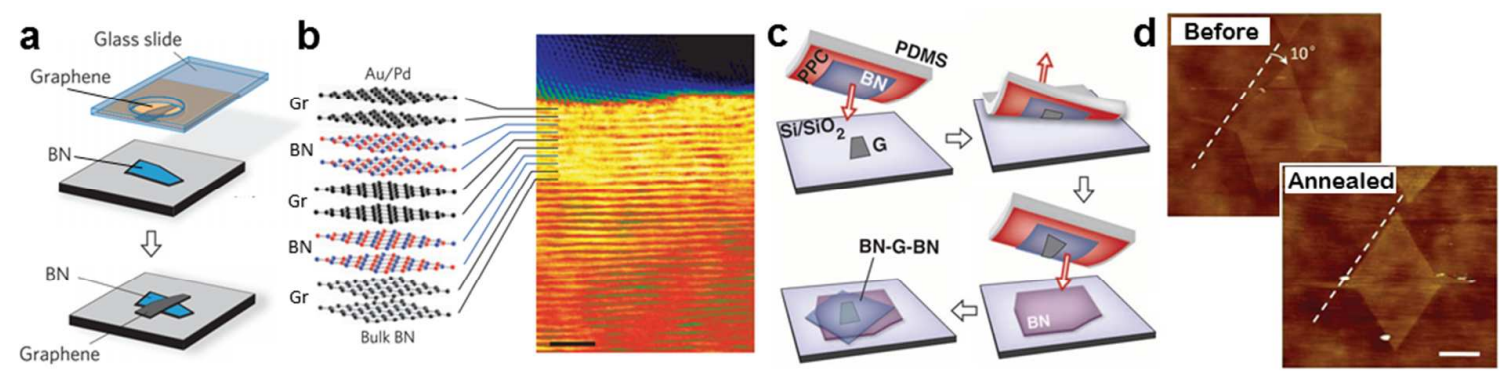

Figure 3. (a) Schematic of the alignment and stacking of a graphene flake on $h-B N$ with the aid of a micromanipulator. (b) Sectional STEM image and schematic of a heterostructure fabricated by the sequential stacking of graphene and $h-\mathrm{BN}$ layers. (c) Schematics for the polymer-free stacking of 2D materials aided by van der Waals interactions. In this case, graphene is picked up by an $h-B N$ flake, and then both are transferred onto another $h-B N$ flake to encapsulate the graphene. (d) AFM image showing the rotation of a graphene flake on $h-B N$ after annealing at $400^{\circ} \mathrm{C}$. (a) Adapted by permission from Macmillan Publishers Ltd: Nat. Nanotechnol. (ref. 11), copyright 2010. (b) Reprinted by permission from Macmillan Publishers Ltd: Nat. Mater. (ref. 25), copyright 2012. (c) From ref. 29. Reprinted with permission from AAAS. (d) Reprinted figure with permission from ref. 36. Copyright 2016 by the American Physical Society. 
a
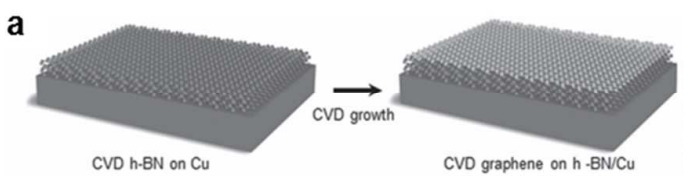

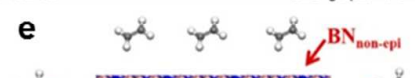

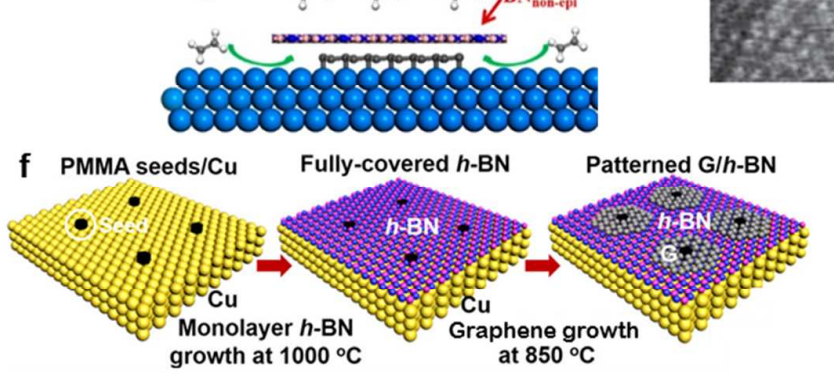

b
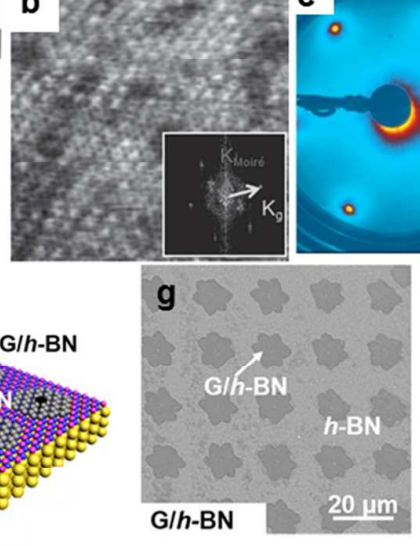
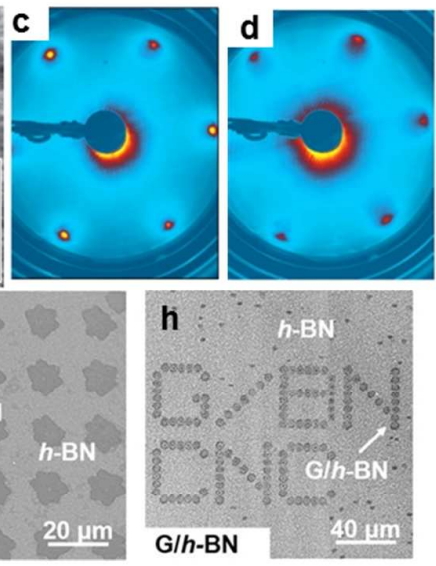

Figure 4. (a) Schematics of the growth of single-layer graphene on $h-\mathrm{BN}$ by CVD. (b) STM image of the graphene grown on $h-\mathrm{BN}$ showing a Moiré pattern with a period of $0.55 \mathrm{~nm}$. Size of the area is $10 \times 10 \mathrm{~nm}^{2}$. Inset is the FFT of the image. (c, d) LEED patterns taken from a layer of $h-\mathrm{BN}$ on $\mathrm{Cu}(111)$ (c) and after the growth of graphene (d). (e) Schematics representing the growth of graphene in the space between $\mathrm{Ni}(111)$ and a disoriented $h-\mathrm{BN}$ layer. (f) Schematics of the growth of patterned graphene/ $h-\mathrm{BN}$ heterostacks on $\mathrm{Cu}$ foils from PMMA seeds. (g, h) SEM images showing patterned domains. (a, b) Reproduced with permission from ref. 59. Copyright 2013, John Wiley and Sons. (c, d) Reprinted with permission from ref. 62. Copyright 2013 American Chemical Society. (e) Reprinted with permission from ref. 67. Copyright 2015 American Chemical Society. (f-h) Reprinted with permission from ref. 69. Copyright 2016 American Chemical Society. 

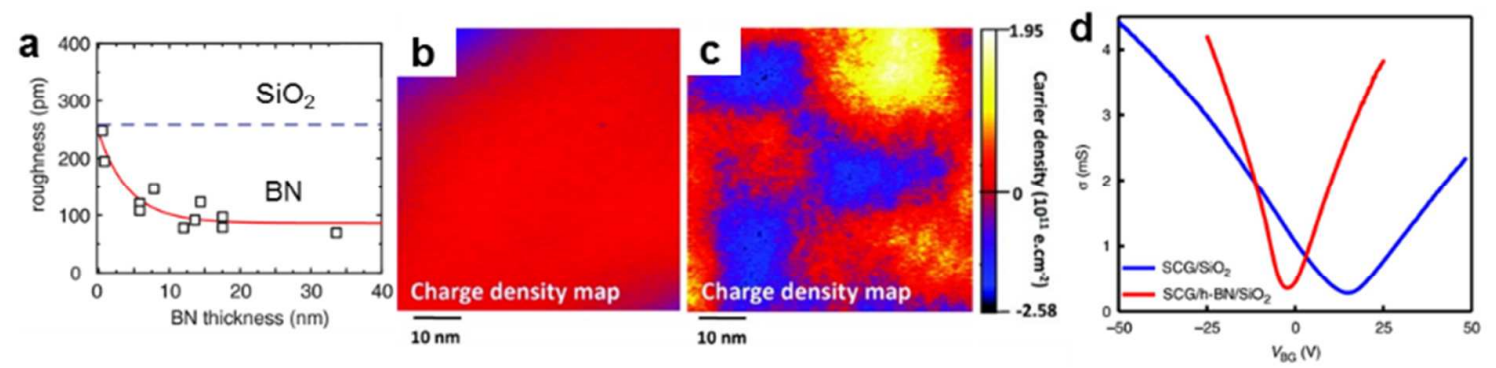

Figure 5. (a) Surface roughness of graphene on $\mathrm{SiO}_{2}$ and on $h-\mathrm{BN}$ with different thicknesses. (b,c) Charge density for graphene on $h-$ $\mathrm{BN}$ (b) and graphene on $\mathrm{SiO}_{2}$ (c). (d) Transfer characteristics of a single-layer graphene FET on $h-\mathrm{BN}$ (red) and on $\mathrm{SiO}_{2}$ (blue). (a) Adapted by permission from Macmillan Publishers Ltd: Nat. Nanotechnol. (ref. 11), copyright 2010. (b,c) Adapted with permission from ref. 102. Copyright 2011 American Chemical Society. (d) Reprinted with permission from ref. 103. 

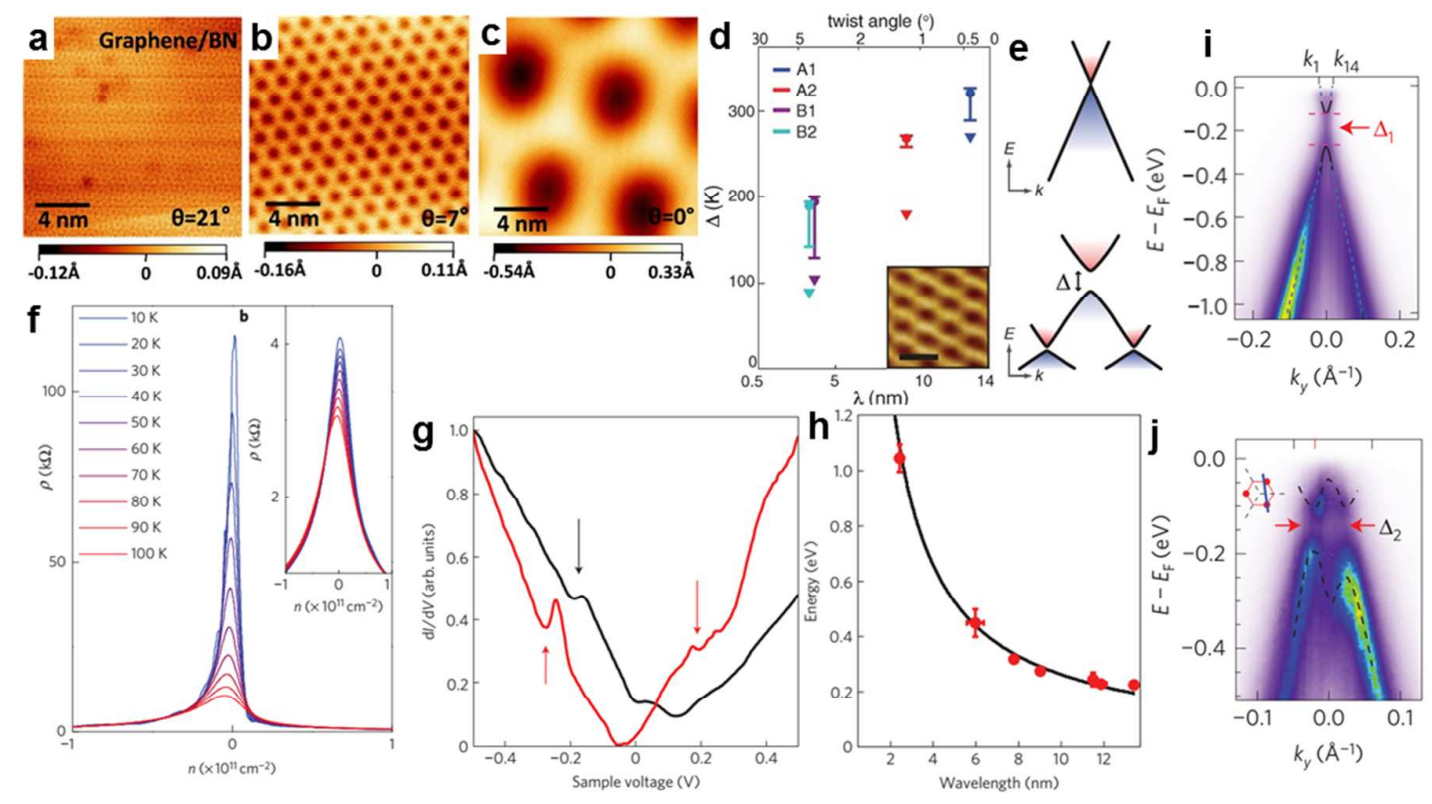

Figure 6. (a-c) Moiré patterns observed by STM for graphene on $h-\mathrm{BN}$ and dependence on the relative orientation between both. The relative orientations are $21^{\circ}(\mathrm{a}), 7^{\circ}$ (b) and $0^{\circ}$ (c). (d) Measured bandgaps for graphene deposited on $h-\mathrm{BN}$ as a function of their relative orientation. $\lambda$ is the wavelength of the Moiré pattern. (e) Schematics of the band structure of graphene (top) and of graphene on top of $h$-BN (bottom), showing the opened band gap $(\Delta)$ and the appearance of secondary Dirac points. (f) Longitudinal resistivity at different temperatures of graphene supported on $h-\mathrm{BN}$ before and after (inset) encapsulation with $h-\mathrm{BN}$. After the encapsulation the resistivity does not increase at lower temperatures, evidencing the disappearance of the gap. (g) STS dl/dV curves for graphene on $h-\mathrm{BN}$ with two different Moiré wavelengths (9.0 nm black curve, $13.4 \mathrm{~nm}$, red curve). The arrows indicate the position of the SDCs. (h) Energy difference between the SDCs and the original Dirac point respect to the Moiré wavelength. (i,j) ARPES measurement of graphene on aligned $h-B N$. (i) Band gap of the Dirac point, observed after doping the sample with rubidium so the gap appears in the ARPES. (j) Band gap at the SDCs. (a-c) Adapted with permission from ref. 102. Copyright 2011 American Chemical Society. (d, e) From ref. 115. Reprinted with permission from AAAS. (f) Reprinted by permission from Macmillan Publishers Ltd: Nat. Phys. (ref. 117), copyright 2014. (g, h) Reprinted by permission from Macmillan Publishers Ltd: Nat. Phys. (ref. 113), copyright 2012. (i, j) Adapted by permission from Macmillan Publishers Ltd: Nat. Phys. (ref. 116), copyright 2016. 

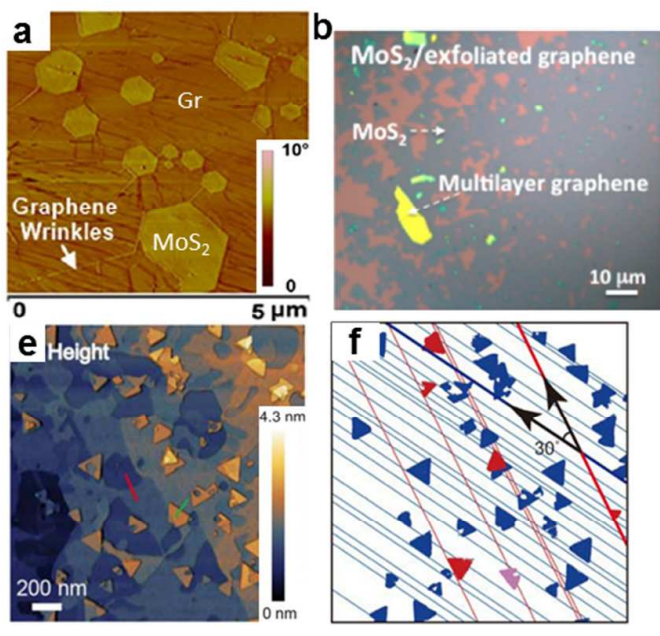
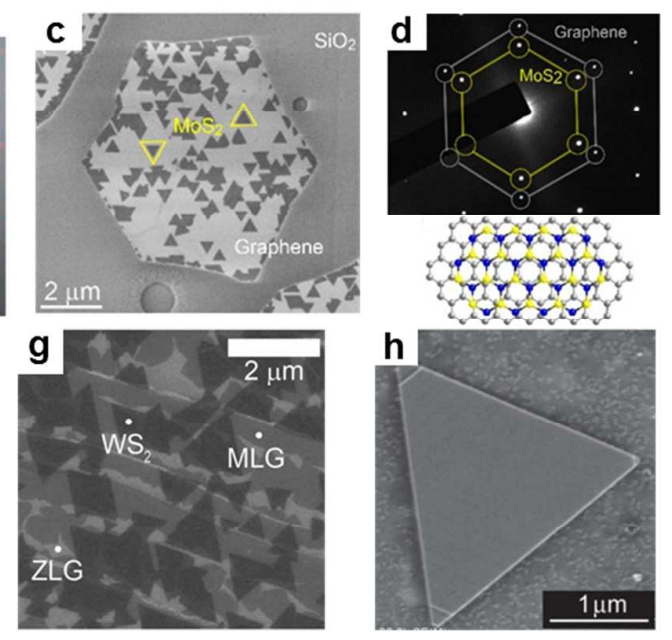

Figure 7. (a) AFM phase image of $\mathrm{MoS}_{2}$ grains grown on graphene/Cu. (b) Optical image of $\mathrm{MoS}_{2}$ grown on exfoliated graphene/SiO by using a seed promoter. (c, d) SEM image (c) and selected area diffraction (d) of $\mathrm{MoS}_{2}$ growth on graphene by CVD. Bottom of (d) shows an atomic model schematic of $\mathrm{MoS}_{2}$ grown on graphene determined by the SAED. (e, f) AFM image of MoS 2 grown on EG (e) and the grain orientation extracted from it (f). (g) SEM image of $\mathrm{WS}_{2}$ flakes grown on EG. (h) SEM image of an $\mathrm{NbS}_{2}$ grain grown on graphene by CVD. (a) Adapted with permission from ref. 70. Copyright 2012 American Chemical Society. (b) Reprinted with permission from ref. 71. Copyright 2014 American Chemical Society. (c, d) Adapted with permission from ref. 72,79. Copyright 2015 \& 2016 American Chemical Society. (e, f) Reprinted with permission from ref. 78. Copyright 2016 American Chemical Society. (g) Adapted with permission from ref. 83. (h) Reproduced from ref. 14 with permission from The Royal Society of Chemistry. 

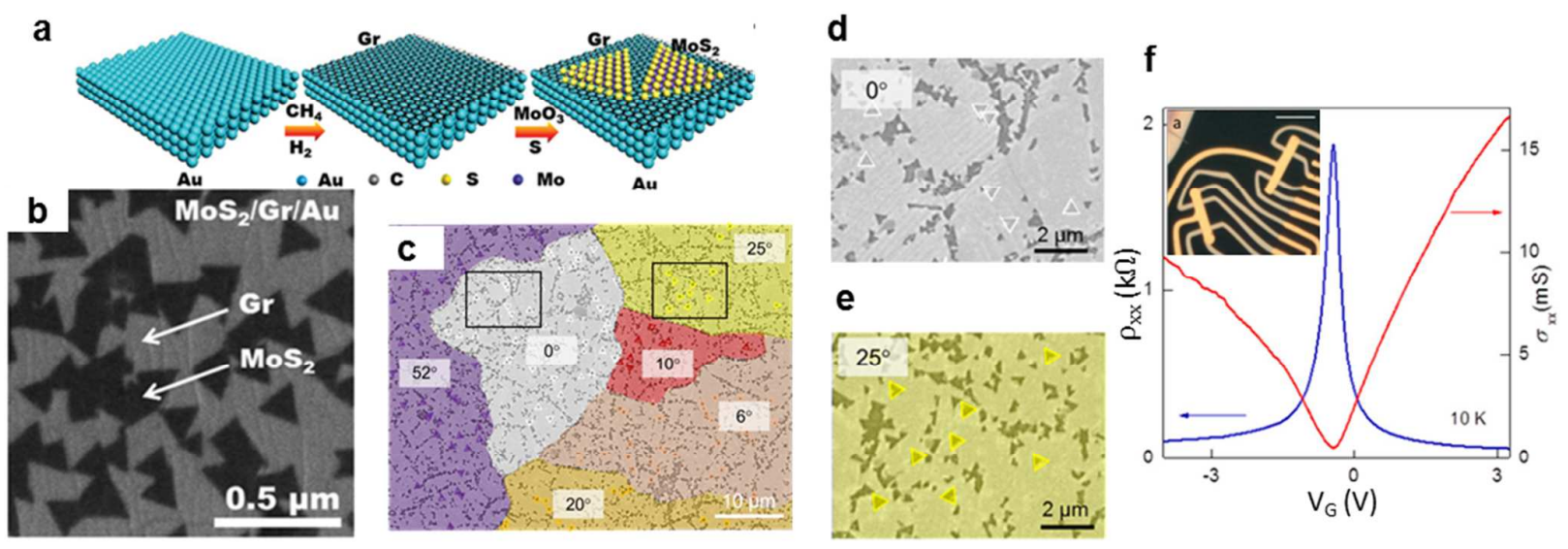

Figure 8. (a) Schematics for the two-step CVD growth of $\mathrm{MOS}_{2}$ on graphene. (b) SEM image of the as-grown MoS 2 /graphene on Au by the two-step CVD. (c-e) Determination of the grain structure of graphene by growing oriented $\mathrm{MoS}_{2}$ grains on it. SEM images showing a large region containing several domains (c) and enlarged images of some grains (d,e). The numbers represent the relative orientations of the grains. (f) Transfer curve of a graphene FET supported on $\mathrm{MoS}_{2}$ and encapsulated with an $h-\mathrm{BN}$ layer serving also as dielectric gate. Inset shows an image of the device. (a,b) Reproduced with permission from ref. 76 . Copyright 2015, John Wiley and Sons. (c-e) Adapted with permission from ref. 79. Copyright 2016 American Chemical Society. (f) Adapted with permission from ref. 28. Copyright 2014 American Chemical Society. 

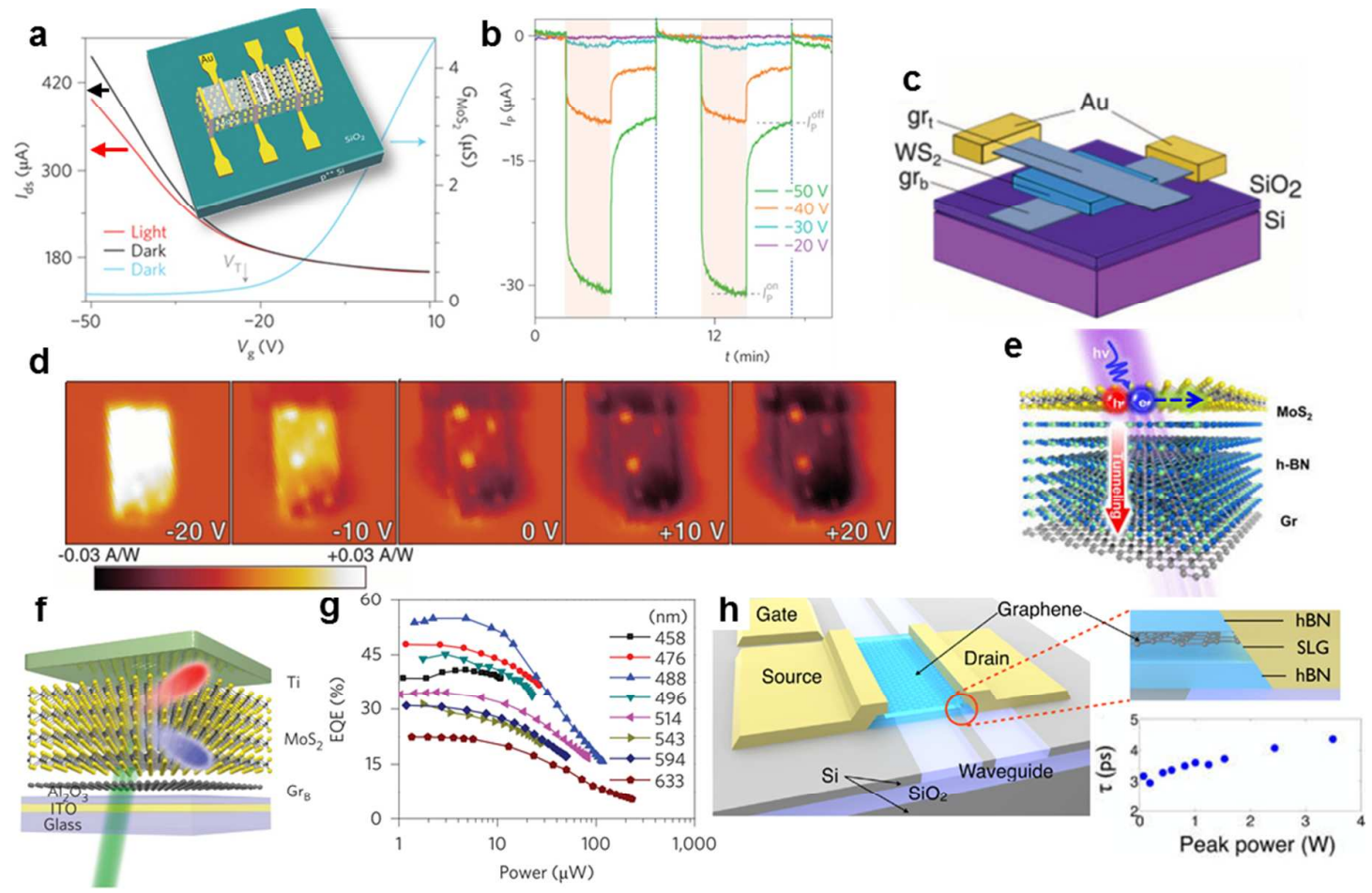

Figure 9. (a) Transfer curve of graphene on $\mathrm{MoS}_{2}$ with (red) and without (black) illumination. Inset is a schematics of the device. (b) Dependence of the photocurrent on the gate voltage for the device shown in (a). Shaded regions correspond to illumination periods. (c) Schematics of a photodetector composed of $\mathrm{WS}_{2}$ encapsulated with graphene electrodes and $h$-BN. (d) Photocurrent maps taken on the device shown in (c) for different gate voltages and without applied bias. Signal is only observed in the region were the layers overlap. (e) Schematic of the $\mathrm{MoS}_{2} / h-\mathrm{BN} /$ graphene photodetector with the $h-\mathrm{BN}$ tunnel barrier to decrease the dark current. $(f, g)$ Schematic of a single-gated graphene/MoS $\mathrm{S}_{2}$ with a metal electrode $(f)$, and laser power dependence of EQE for different excitation wavelengths $(\mathrm{g})$. (h) Schematic of a photodetector composed of graphene encapsulated in $h-\mathrm{BN}$, and coupled to a silicon waveguide. Right bottom inset: dependence of the response time of the graphene autocorrelator to the excitation power. (a,b) Adapted by permission from Macmillan Publishers Ltd: Nat. Nanotechnol. (ref. 39), copyright 2013. (c,d) From ref. 42. Adapted with permission from AAAS. (e) Reprinted with permission from ref. 128. Copyright 2017 American Chemical Society. (f,g) Reprinted by permission from Macmillan Publishers Ltd: Nat. Nanotechnol. (ref. 43), copyright 2013. (h) Adapted with permission from ref. 129. Copyright 2015 American Chemical Society. 


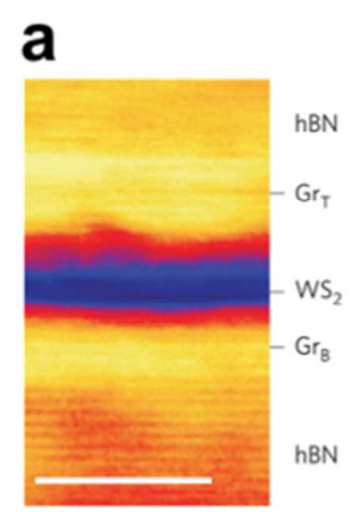

b
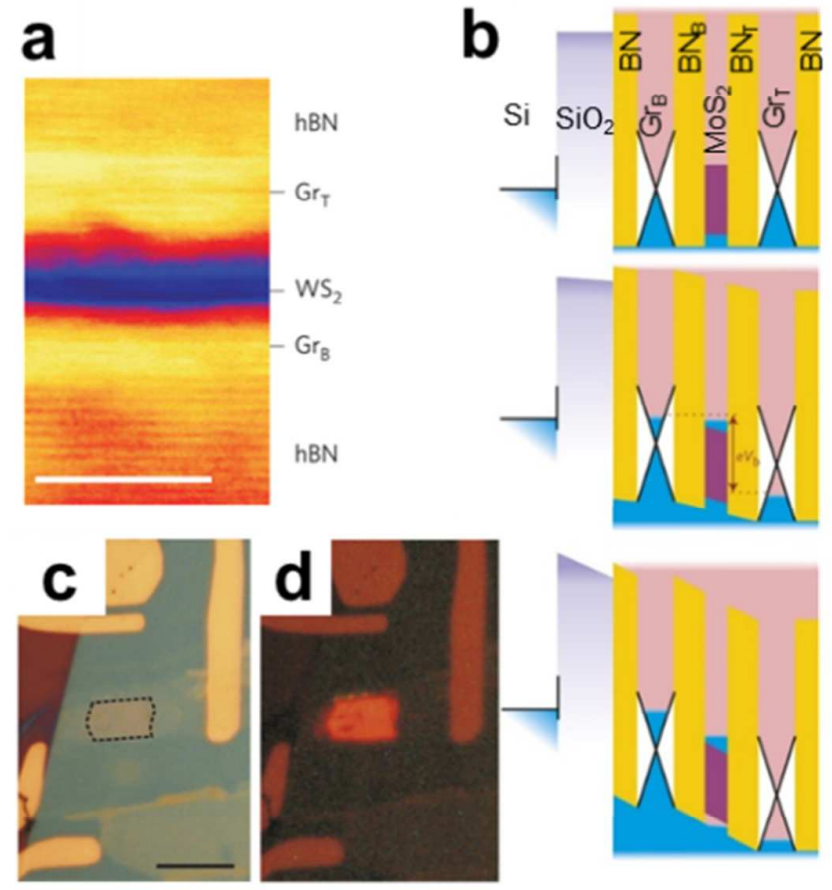

e

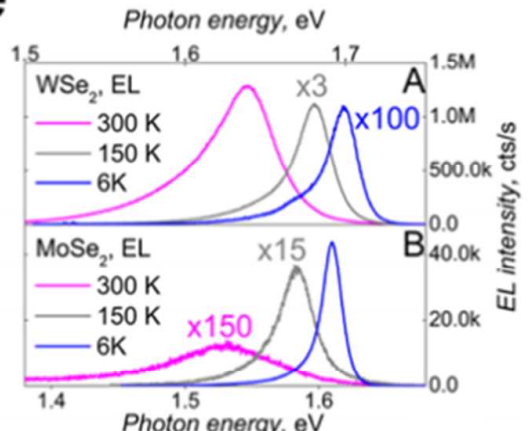

f

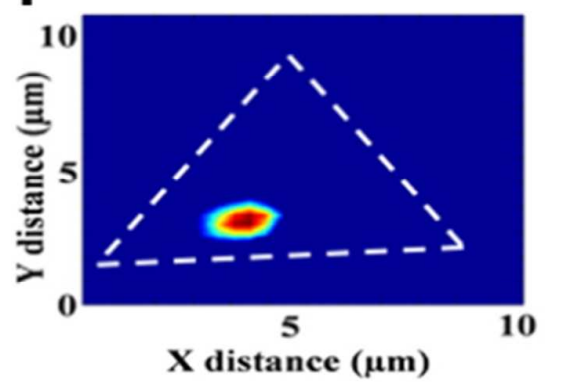

Figure 10. (a) False colored STEM image of a LED composed of a heterostructure with a WS 2 QW separated by $h-B N$ layers from the graphene electrodes. (b) Band structure of a LED composed of a single QW for zero (top), intermediate (middle) and high (bottom) applied bias. (c,d) Optical images of a working LED composed of a single QW in the off (c) and on (d) states. (e) Electroluminescence of heterostructure LEDs with WSe $\mathrm{S}_{2}$ (top) and $\mathrm{MoSe}_{2}$ (bottom) QWs measured at several temperatures, evidencing the increased electroluminescence with the temperature for the heterostructures with the $\mathrm{WSe}_{2} \mathrm{QW}$. (f) Localized electroluminescence from defects on WSe $\mathrm{W}_{2}$ on a LED heterostructure. (a-d) Adapted by permission from Macmillan Publishers Ltd: Nat. Mater. (ref. 133), copyright 2015. (e) Reprinted with permission from ref. 134. Copyright 2015 American Chemical Society. (f) Reprinted with permission from ref. 136. Copyright 2016 American Chemical Society. 

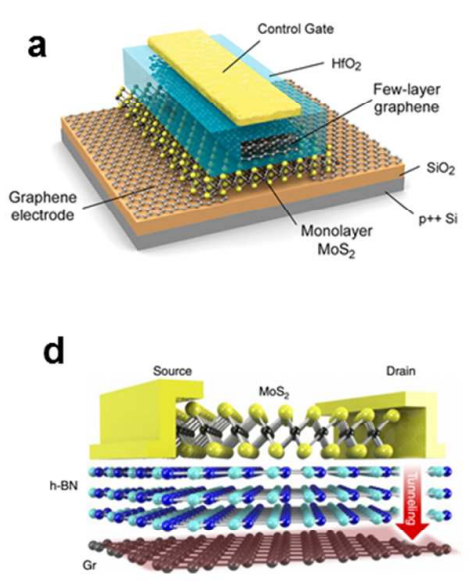
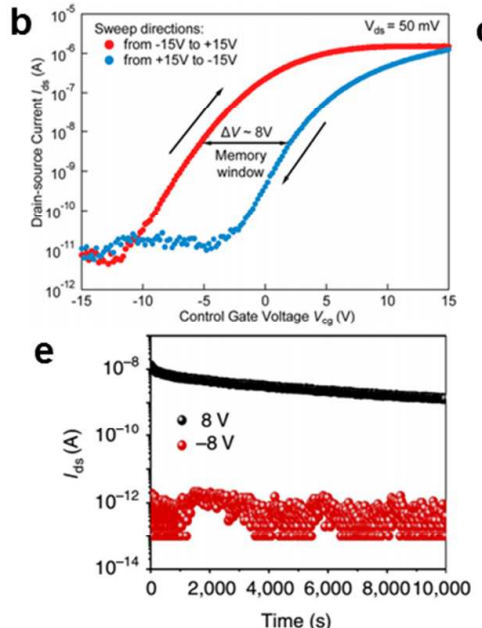
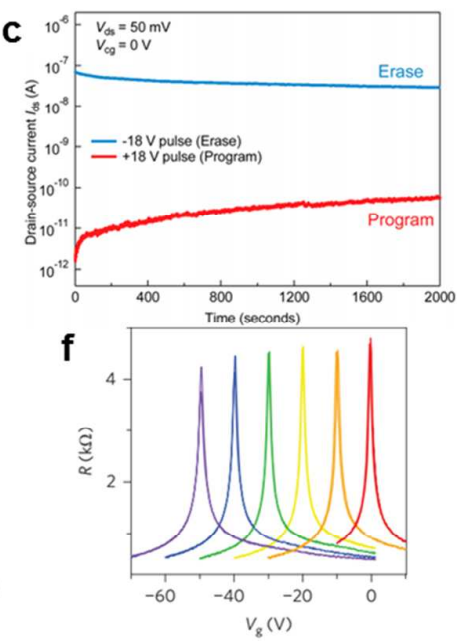

Figure 11. (a) Schematics of a floating-gate memory, with a graphene electrode, a $\mathrm{MoS}_{2}$ channel and a graphene floating-gate. (b) Transfer characteristics of the device in (a) collected in two different sweep directions for the gate. The hysteresis is a consequence of the charge accumulated in the graphene floating-gate. (c) Time dependence of the drain currents in the program and erase states. (d) Schematics of a two-terminal memory consisting of a $\mathrm{MoS}_{2}$ channel and a graphene floating gate separated by $h-B N$. (e) Time dependence of the program and erase states in device shown in (d). (f) Transfer characteristics of a memory based on the light-induced doping of graphene on $h-\mathrm{BN}$. The red trace is the pristine device, while the subsequent traces were collected after photodoping with different applied gates. (a-c) Reprinted with permission from ref. 40. Copyright 2013 American Chemical Society. (d,e) Reprinted with permission from ref. 140. (f) Reprinted by permission from Macmillan Publishers Ltd: Nat. Nanotechnol. (ref. 141), copyright 2014. 

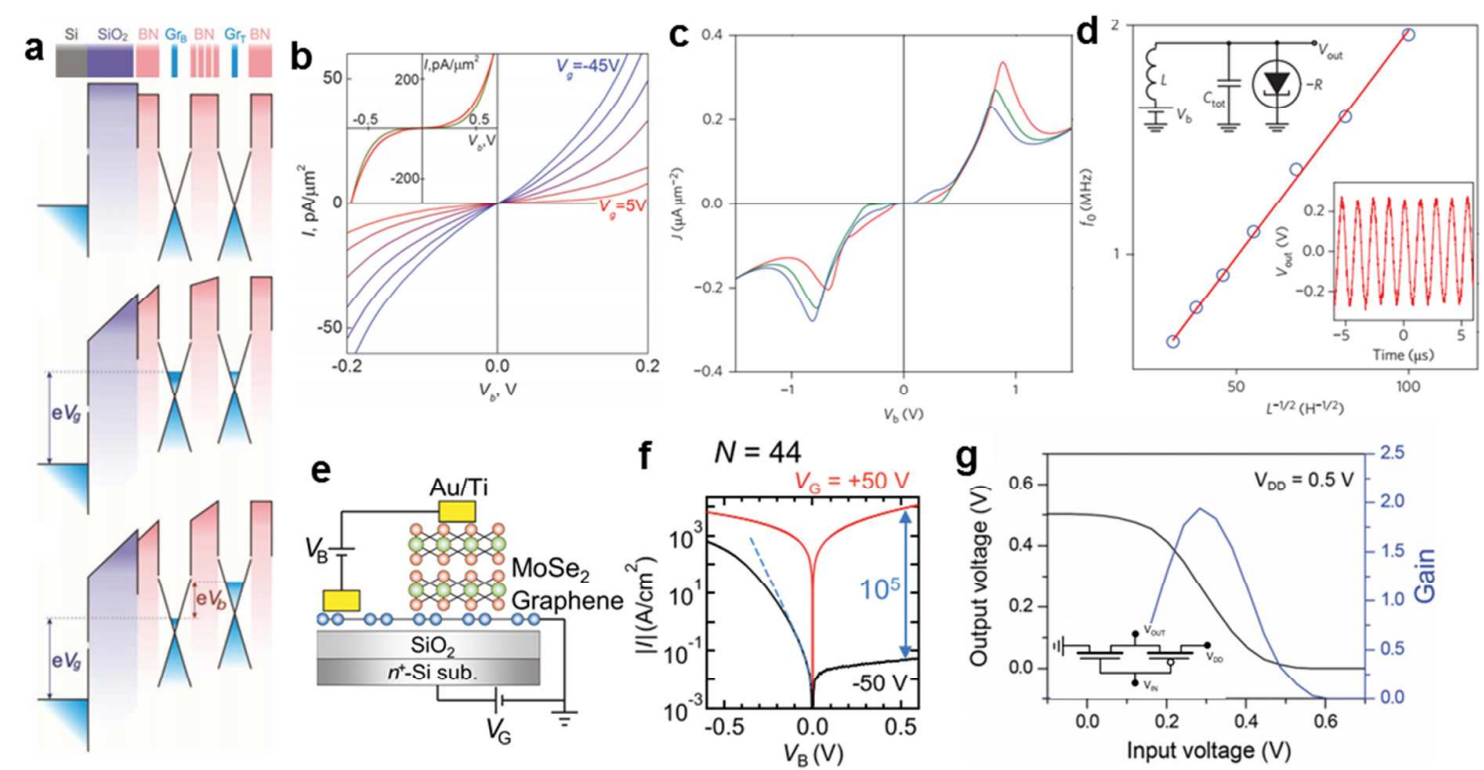

Figure 12. (a) Schematics of a graphene field-effect tunnel transistor (top) and band structure for zero gate and zero bias between the two graphene layers (top), for an applied gate (middle) and for applied bias and gate (bottom). (b) Tunneling current of a device such as that of (a) for different gate voltages. (c) Bias dependence of the current density of a tunnel VFET for large applied bias, and for different applied gates (+40, 0 and $-40 \mathrm{~V}$ for the red, green and blue curves respectively). (d) Schematics of a RF oscillator fabricated with a resonant VFET, and resonant frequency dependence on the inductance of the LC circuit. (e,f) Schematics (e) and I-V characteristics of a graphene/MoSe $/$ Ti vertical heterostructure. An on/off ratio of $10^{5}$ is achieved by tuning the gate voltage from +50 to $-50 \mathrm{~V}$. (g) Output and gain of an inverter device by combining $\mathrm{n}$-type (graphene/MoS $\mathrm{S}_{2}$ ) and ptype (WSe ${ }_{2}$ ) vertical FETs. (a,b) From ref. 24. Reprinted with permission from AAAS. (c,d) Adapted by permission from Macmillan Publishers Ltd: Nat. Nanotechnol. (ref. 147), copyright 2014. (e,f) Reprinted from ref. 149, with the permission of AIP Publishing. (g) Reproduced with permission from ref. 150. Copyright 2016, John Wiley and Sons. 

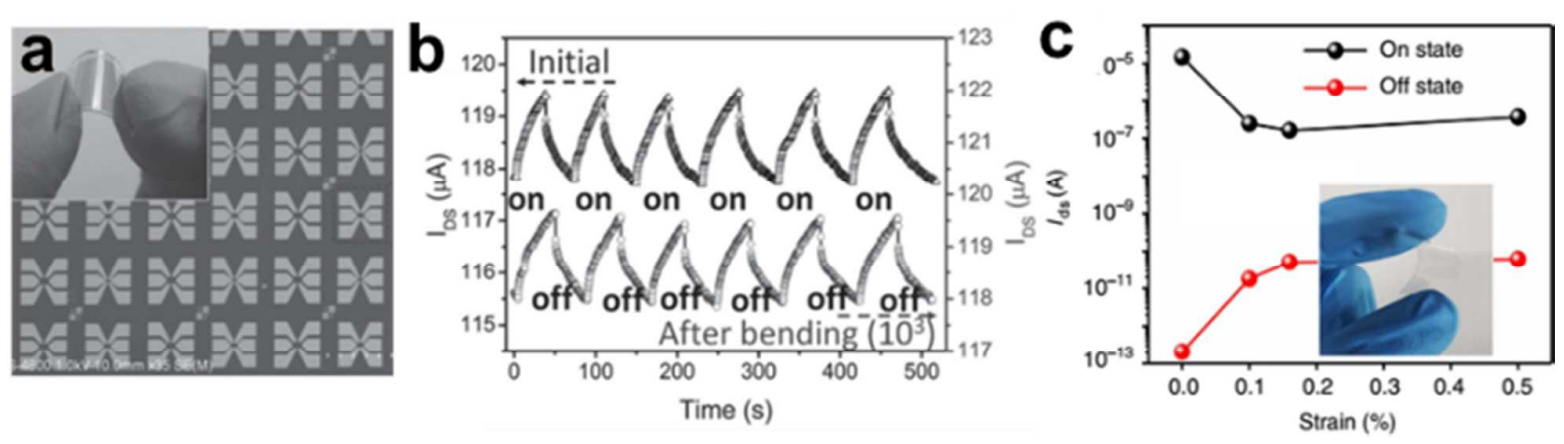

Figure 13. (a) SEM image of graphene/ $\mathrm{MoS}_{2}$ phototransistors on flexible PET. Inset: photograph of the sample. (b) Time traces of the drain current with light on and off measured before (top) and after (bottom) a bending test of 1000 cycles. (c) Flexible memory composed of a graphene heterostructures supported on PET, and strain dependence of the on and off currents. (a,b) Reproduced with permission from ref. 124. Copyright 2014, John Wiley and Sons. (c) Adapted with permission from ref. 140. 

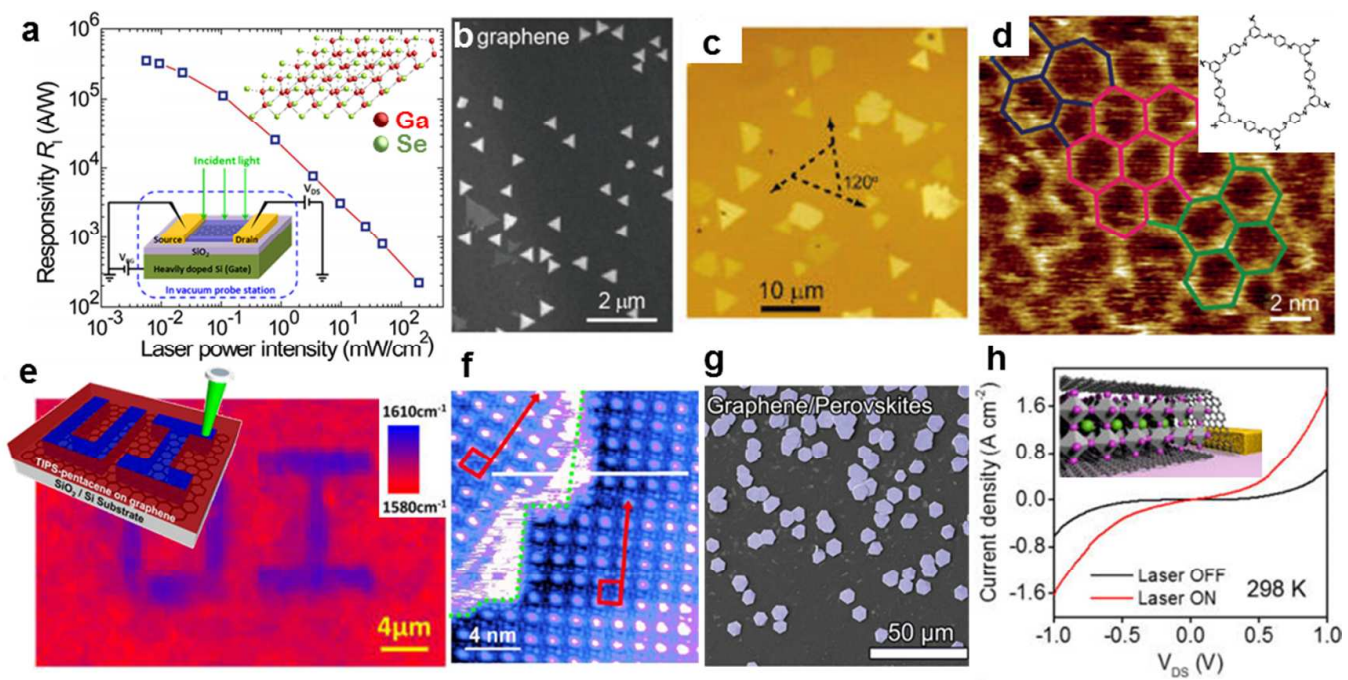

f
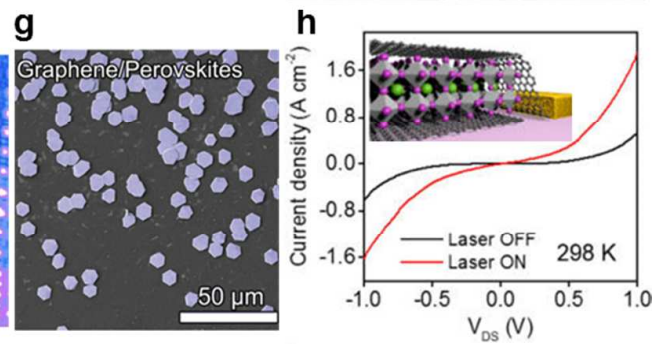

Figure 14. (a) Schematics of a graphene/GaSe FET, and photoresponsivity dependence on the laser power intensity. (b) SEM image of $\mathrm{In}_{2} \mathrm{Se}_{3}$ flakes grown on graphene. (c) Optical image of $\mathrm{Bi}_{2} \mathrm{Se}_{3}$ grown on graphene. (d) STM image of COF ${ }_{B T A-P D A}$ on graphene/Cu. (e) Schematic for laser writing of $p$-n junctions on TIPS-pentacene/graphene, and mapping of Raman $G$ band wavelength after writing "UI". (f) STM image of an assembled film of phthalocyanine on graphene/Cu(100), showing a boundary of different assembled regions. (g) False colored SEM image of a perovskite grown on graphene. (h) I-V curves with and without external illumination, of a device consisting of a perovskite encapsulated in two graphene electrodes. (a) Adapted with permission from ref. 156. (b) Reprinted with permission from ref. 94. Copyright 2013 American Chemical Society. (c) Reprinted with permission from ref. 93. Copyright 2010 American Chemical Society. (d) Adapted with permission from ref. 159. Copyright 2014, John Wiley and Sons. (e) Adapted with permission from ref. 162. Copyright 2014 American Chemical Society. (f) Reprinted with permission from ref. 164. Copyright 2013 American Chemical Society. (g) Reproduced with permission from ref. 97. Copyright 2015, John Wiley and Sons. (h) Adapted with permission from ref. 166. Copyright 2016 American Chemical Society. 

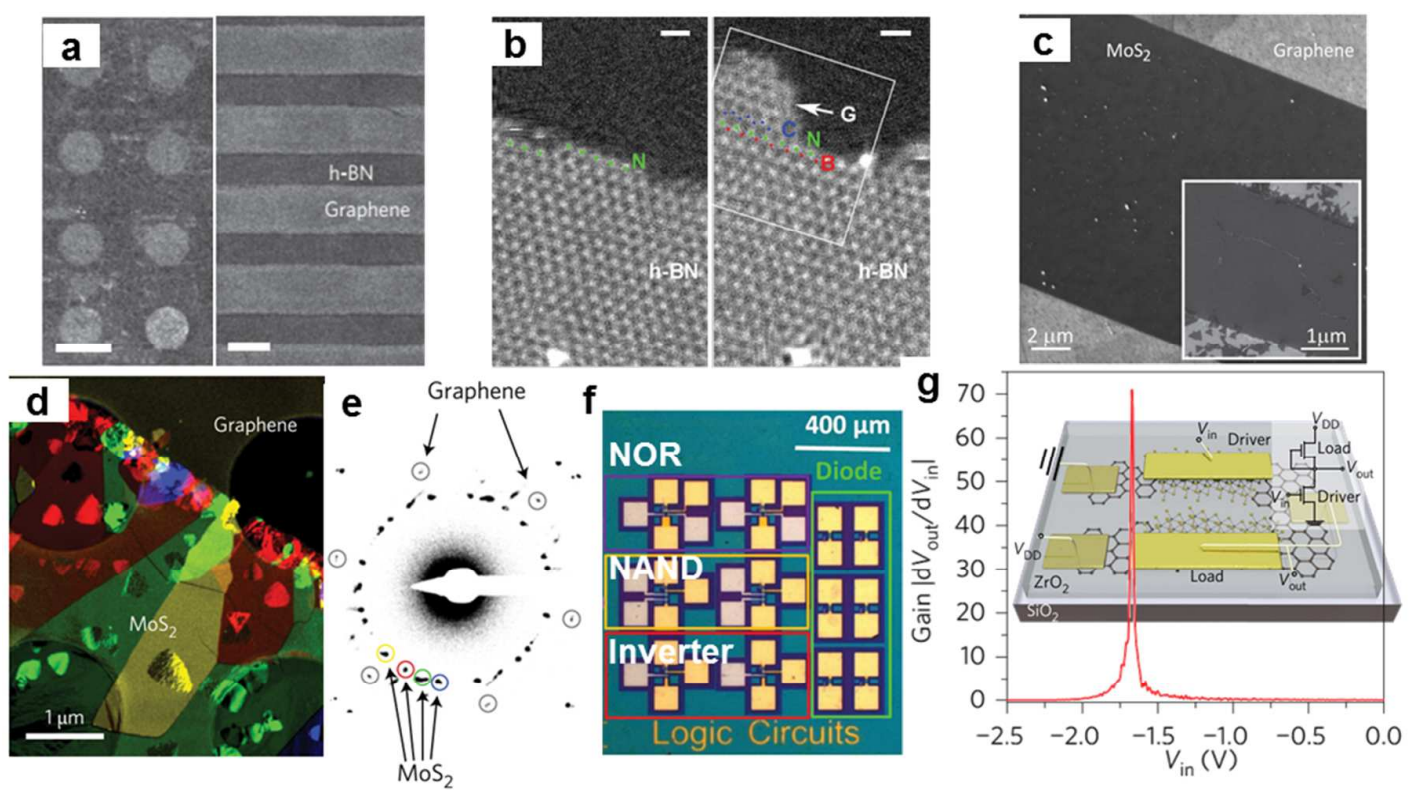

Figure 15. (a) SEM images of patterned $h$-BN/graphene in-plane heterostructures. Graphene circles (left, scale $50 \mu \mathrm{m}$ ) and lines (right, scale $10 \mu \mathrm{m}$ ) on $h-\mathrm{BN}$. (b) Lateral growth of graphene from an N-terminated edge of $h-\mathrm{BN}$ in a TEM chamber. Left and right sides correspond to before and after the graphene growth. (c) SEM images of patterned in-plane MoS $_{2} /$ graphene heterostructures. Inset shows the result for larger Mo pressure, resulting in some $\mathrm{MoS}_{2}$ grains growing also on top of the graphene. (d, e) False colored DF-TEM of polycrystalline $M_{2} S_{2}$ grown from an edge of graphene (d), and the correspondent diffraction pattern showing the single crystal nature of the graphene $(e)$. ( $f, g)$ In-plane logic circuits made from stitched graphene and $\mathrm{MoS}_{2}$. $(\mathrm{g}$ ) Logic inverter from two ntype graphene/MoS 2 lateral transistors. (a) Adapted by permission from Macmillan Publishers Ltd: Nat. Nanotechnol. (ref. 169), copyright 2013. (b) Adapted with permission from ref. 172. (c) Reprinted with permission from ref. 175. Copyright 2016 American Chemical Society. (d,e,g) Adapted by permission from Macmillan Publishers Ltd: Nat. Nanotechnol. (ref. 174), copyright 2016. (f) Adapted with permission from ref. 173. Copyright 2016, John Wiley and Sons. 

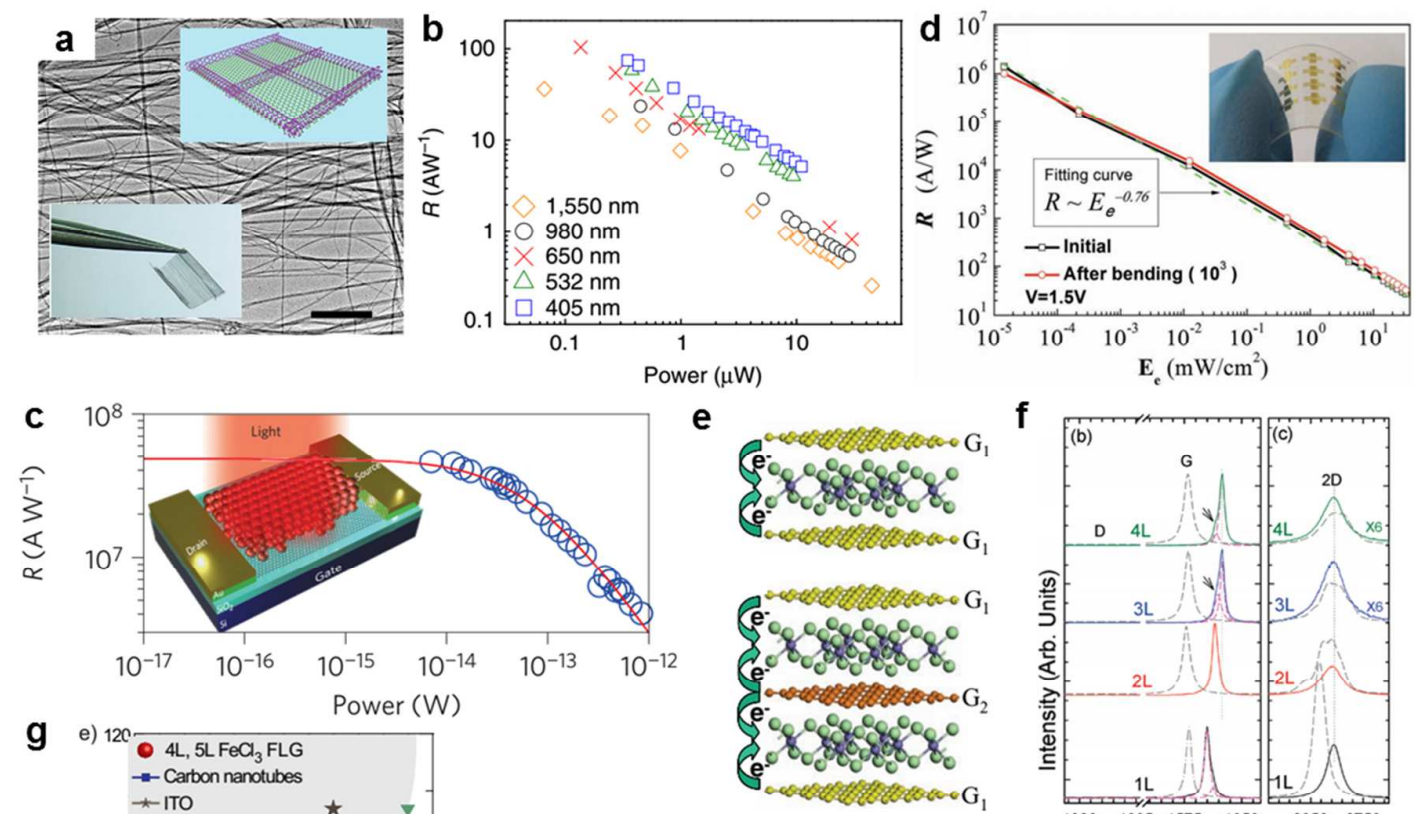

e

h

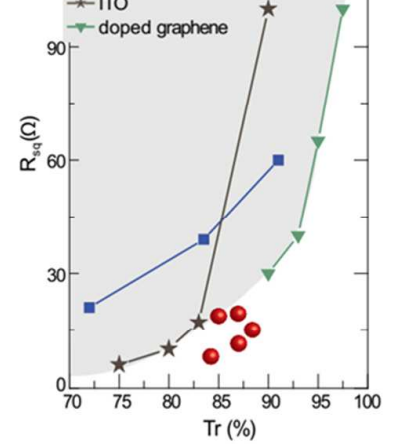

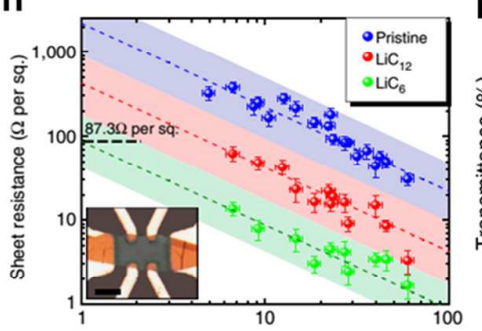

No. of layers (ML)

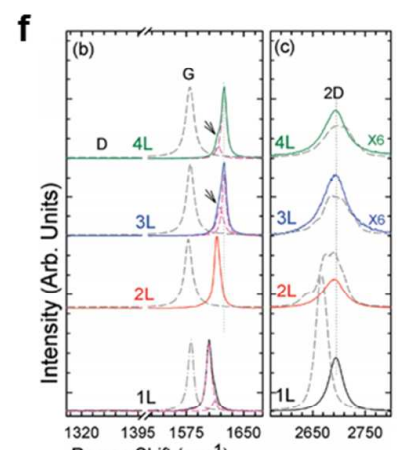

i

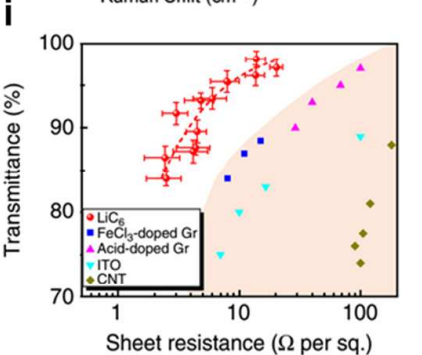

Figure 16. (a) TEM image of a graphene/CNT heterostructure (rebar). Top inset: schematic of the heterostructure. Bottom inset: handling of a free-standing heterostructure. (b) Responsivity of a graphene/CNT heterostructures for different light wavelengths. (c) Illumination power dependence of responsivity for a graphene transistor with a PbS deposited film. (d) Responsivity of a graphene device with deposited $\mathrm{PbS}$ before and after a bending test of 1000 cycles. (e) Schematics of FeCl $\mathrm{S}_{3}$ intercalation in double- (top) and triple-layer (bottom) graphene. (f) Raman spectra of pristine graphene (dotted lines) and that intercalated with $\mathrm{FeCl}_{3}$ (continuous lines) for different flake thicknesses. $(\mathrm{g})$ Sheet resistance versus optical transmittance of $4 \mathrm{~L}$ and $5 \mathrm{~L}$ graphene intercalated with $\mathrm{FeCl}_{3}$, compared with those of ITO and CNTs. (h) Sheet resistance versus number of layers for few-layer pristine graphene and after different stages of intercalation with Li. (i) Sheet resistance versus optical transmittance at $550 \mathrm{~nm}$ of Li-intercalated few-layer graphene compared with values for CNTs, ITO and acid-doped graphene. (a) Adapted by permission from Macmillan Publishers Ltd: Nat. Commun. (ref. 177), copyright 2013. (b) Reprinted with permission from ref. 181. (c) Adapted by permission from Macmillan Publishers Ltd: Nat. Nanotechnol. (ref. 183), copyright 2012. (d) Reproduced with permission from ref. 185. Copyright 2012, John Wiley and Sons. (e) Adapted with permission from ref. 192. Copyright 2010, John Wiley and Sons. (f) Adapted with permission from ref. 193. Copyright 2011 American Chemical Society. (g) Reproduced with permission from ref. 194. Copyright 2012, John Wiley and Sons. (h,i) Reprinted by permission from Macmillan Publishers Ltd: Nat. Commun. (ref. 197), copyright 2014. 


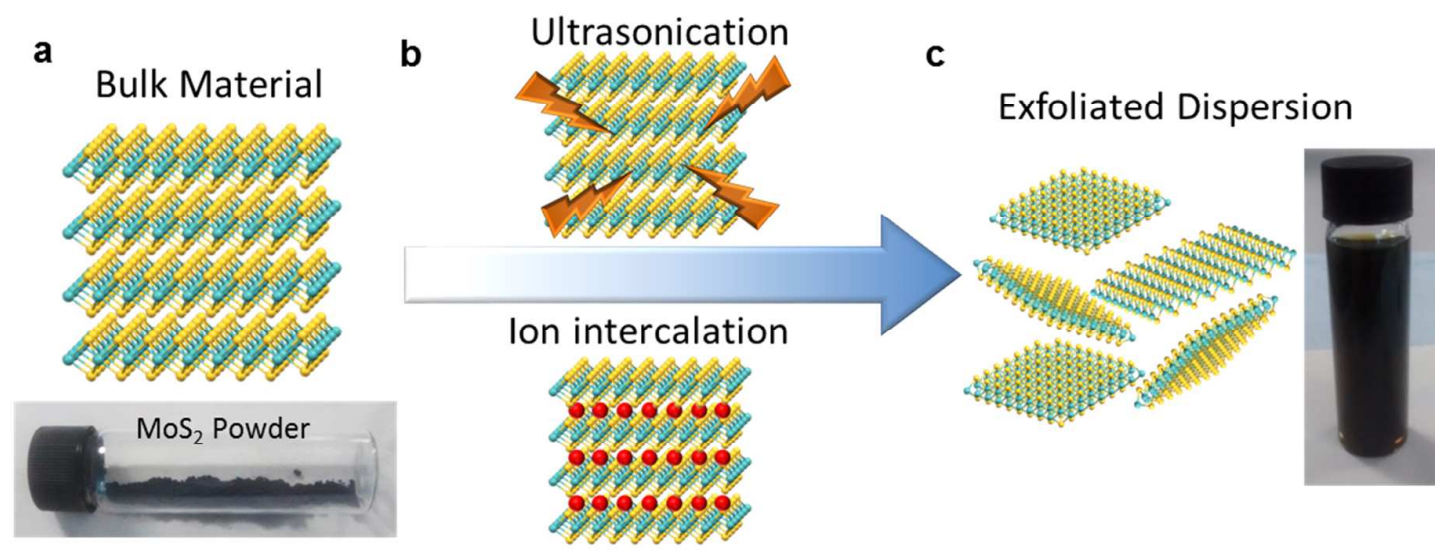

Figure 17. Overview of liquid exfoliation techniques. (a) Crystal structure of a bulk layered 2D materials $\left(\mathrm{MoS}_{2}\right)$ along with a photograph showing the bulk $\mathrm{MoS}_{2}$ powder. (b) Schematic showing two common liquid exfoliation techniques, direct ultrasonication in solvent, and ion intercalation. Both of these techniques are easily scalable and able to produce large amounts of exfoliated dispersed 2D materials. (c) Crystal structure of solvent-stabilised exfoliated $\mathrm{MoS}_{2}$ along with a photograph showing a $\mathrm{MoS}_{2}$ dispersion prepared by ultrasonication in NMP. 


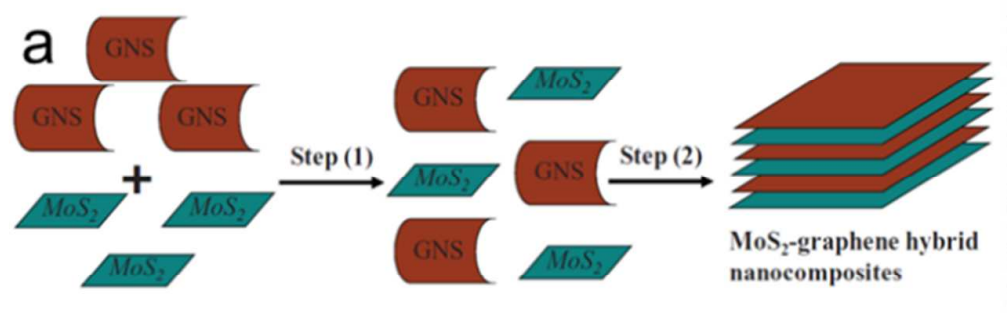

Step (1): Mixed under the assistance of ultrasonication

Step (2): Filtration under the assistance of vacuum

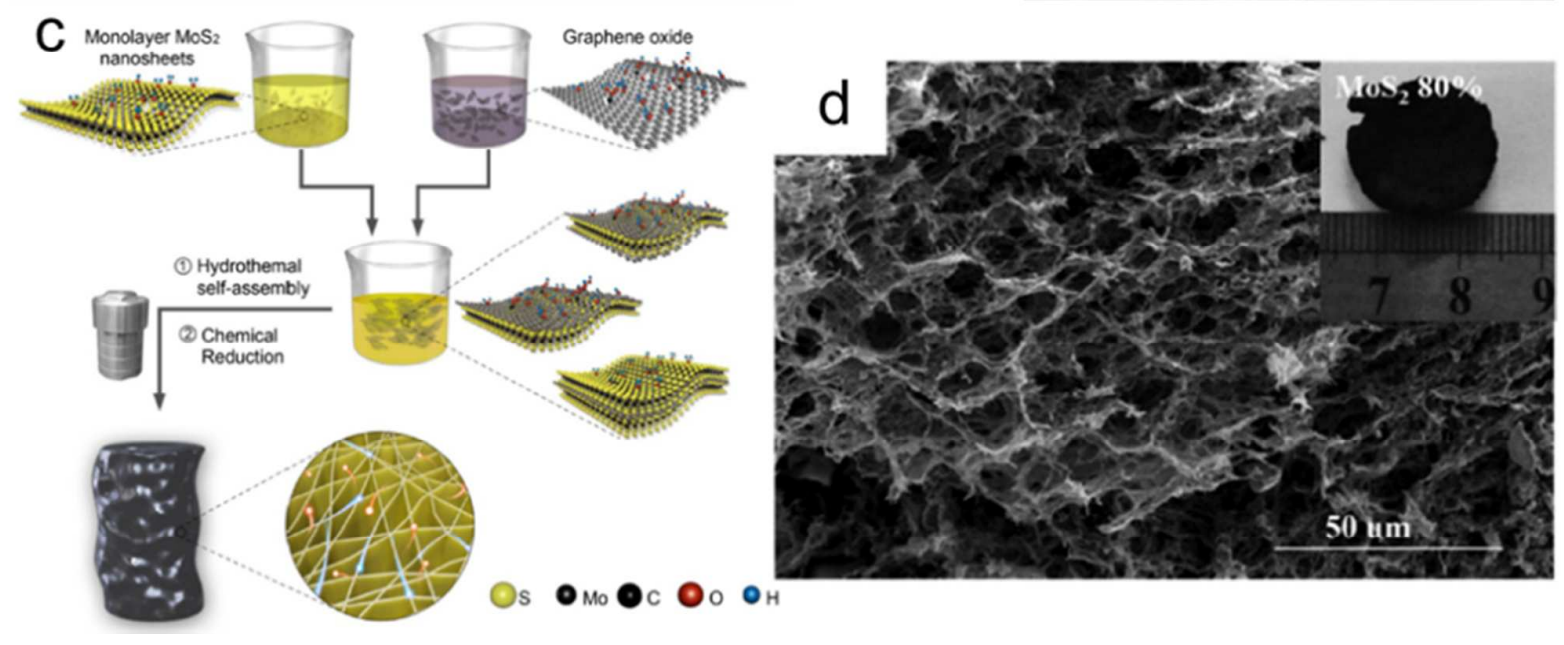

Figure 18. Comparison of hybrid composite synthesis procedures. (a) Schematic illustration showing the formation of a graphene nanosheets (GNS) and $\mathrm{MoS}_{2}$ composite by first mixing with ultrasonication and then forming a film by vacuum filtration. (b) SEM image showing a cross-section of the resultant $\mathrm{MoS}_{2}$-graphene hybrid composite with a laminar structure. $(\mathrm{a}, \mathrm{b})$ Modified from Hu et al.211 (c) Schematic illustration showing the formation of a MoS2/GO hybrid composite aerogel through hydrothermal self-assembly. (d) SEM image showing the morphology of the resulting MoS2/GO porous aerogel composite. Inset shows a photograph of the composite structure, the ratio of MoS2:GO is 80:20. (c,d) Adapted with permission from ref. 220. Copyright 2016 American Chemical Society. 

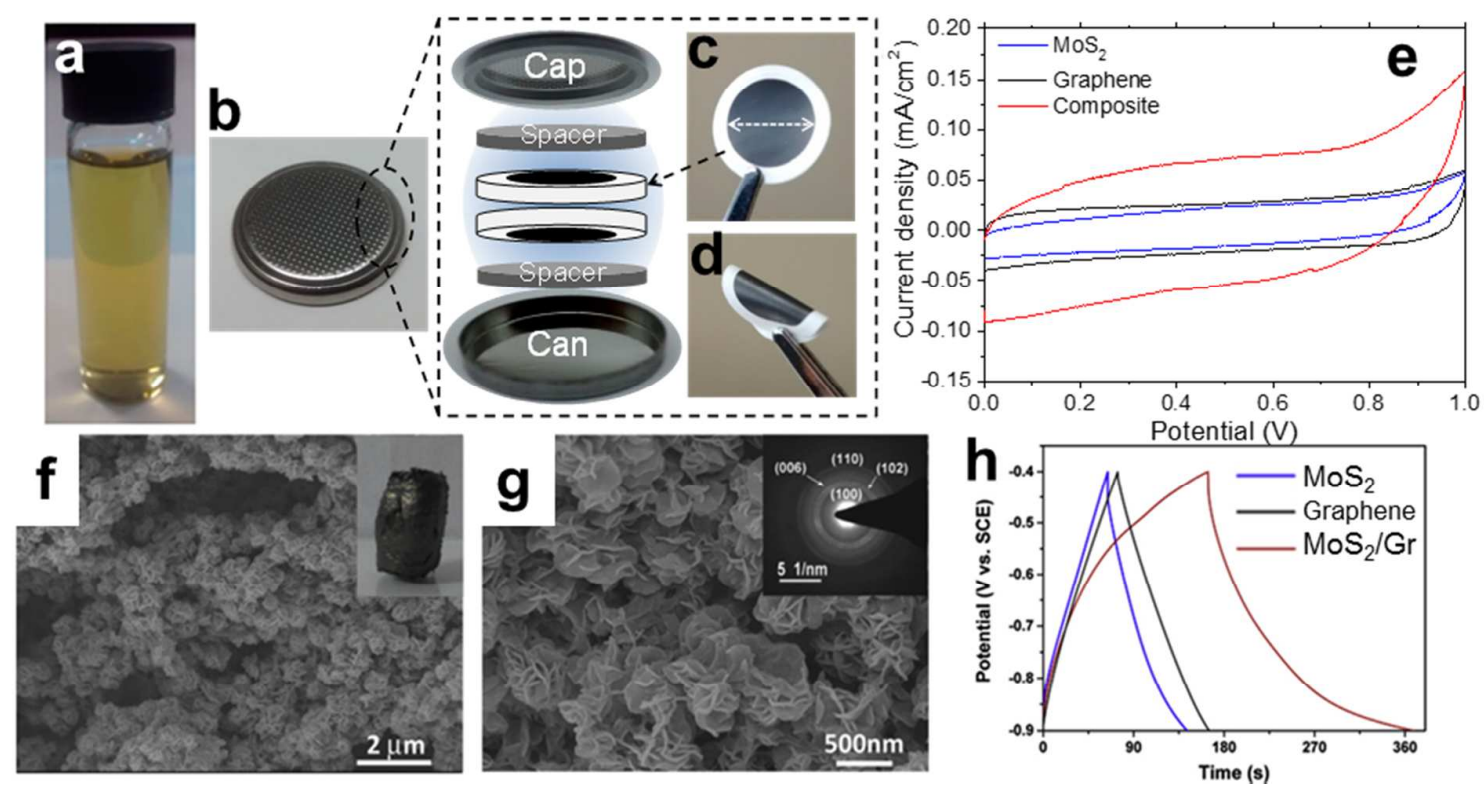

Figure 19. Examples of $\mathrm{MoS}_{2}$ /graphene hybrid composite supercapacitors. (a) Photograph showing a dispersion of exfoliated $\mathrm{MoS}_{2}$ flakes along with a schematic (b) showing the exploded view of an assembled symmetrical coin-cell supercapacitor. Photographs (c,d) show the $\mathrm{MoS}_{2} /$ graphene composite electrodes formed after filtration onto a supporting polymer membrane. (e) Comparison of the electrochemical performance of the single component $\mathrm{MoS}_{2}$, graphene, and the $\mathrm{MoS}_{2}$ /graphene composite illustrating the increased capacitance. Low (f) and high magnification (g) SEM images showing the morphology of a $\mathrm{MoS}_{2}$ /graphene aerogel structure. (h) Comparison of the supercapacitor charge/discharge behaviour again demonstrating the enhanced performance of the $\mathrm{MoS}_{2} /$ graphene composite. (a-e) Adapted with permission from ref. 215. (fh) Adapted from ref. 240, Copyright 2016, with permission from Elsevier. 

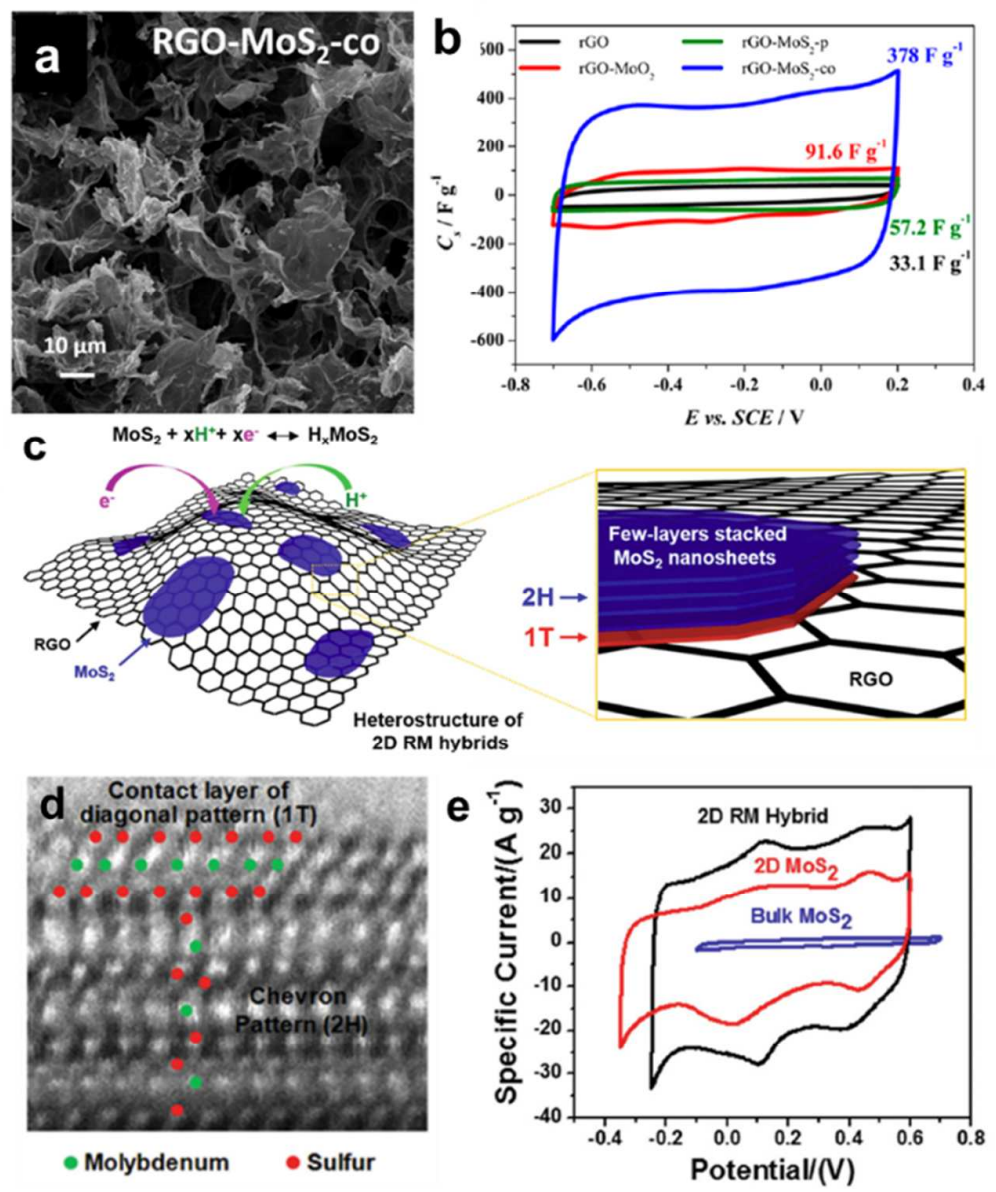

Figure 20. Hybrid composites of mixed $1 \mathrm{~T}-2 \mathrm{H}$ phase $\mathrm{MoS}_{2}$ /graphene for supercapacitors. (a) SEM image showing the morphology of a rGO-MoS ${ }_{2}$ composite that was formed by hydrothermal co-synthesis. The $\mathrm{MoS}_{2}$ is a mixture of both 1T and $2 \mathrm{H}$ phases. (b) Comparison of the electrochemical capacitance for each of the single component electrodes as well as the composite material demonstrating the enhanced performance of the co-synthesised composite which has the mixed phase structure. (c) Schematic diagram showing a $\mathrm{MoS}_{2} /$ graphene heterostructure formed via hydrothermal synthesis illustrating the phase transition that occurs for the $\mathrm{MoS}_{2}$ directly in contact with the $\mathrm{GGO}$. The charge storage mechanism is also illustrated where the $\mathrm{MoS}_{2}$ hybrid undergoes reversible proton $\left(\mathrm{H}^{+}\right)$adsorption. (d) Cross sectional HAADF-STEM image showing the interface between the $\mathrm{MoS}_{2}$ and rGO layers, where the layer directly in contact shows a 1T arrangement while those away from the interface are $2 \mathrm{H}$. (e) Comparison of the electrochemical capacitance for the composite compared to the bulk and exfoliated pure $\mathrm{MoS}_{2}$ illustrating the enhanced capacitance of the hybrid structure. (a,b) Adapted with permission from ref. 238. Copyright 2016 American Chemical Society. (c-e) Adapted with permission from ref. 256. Copyright 2016, John Wiley and Sons. 

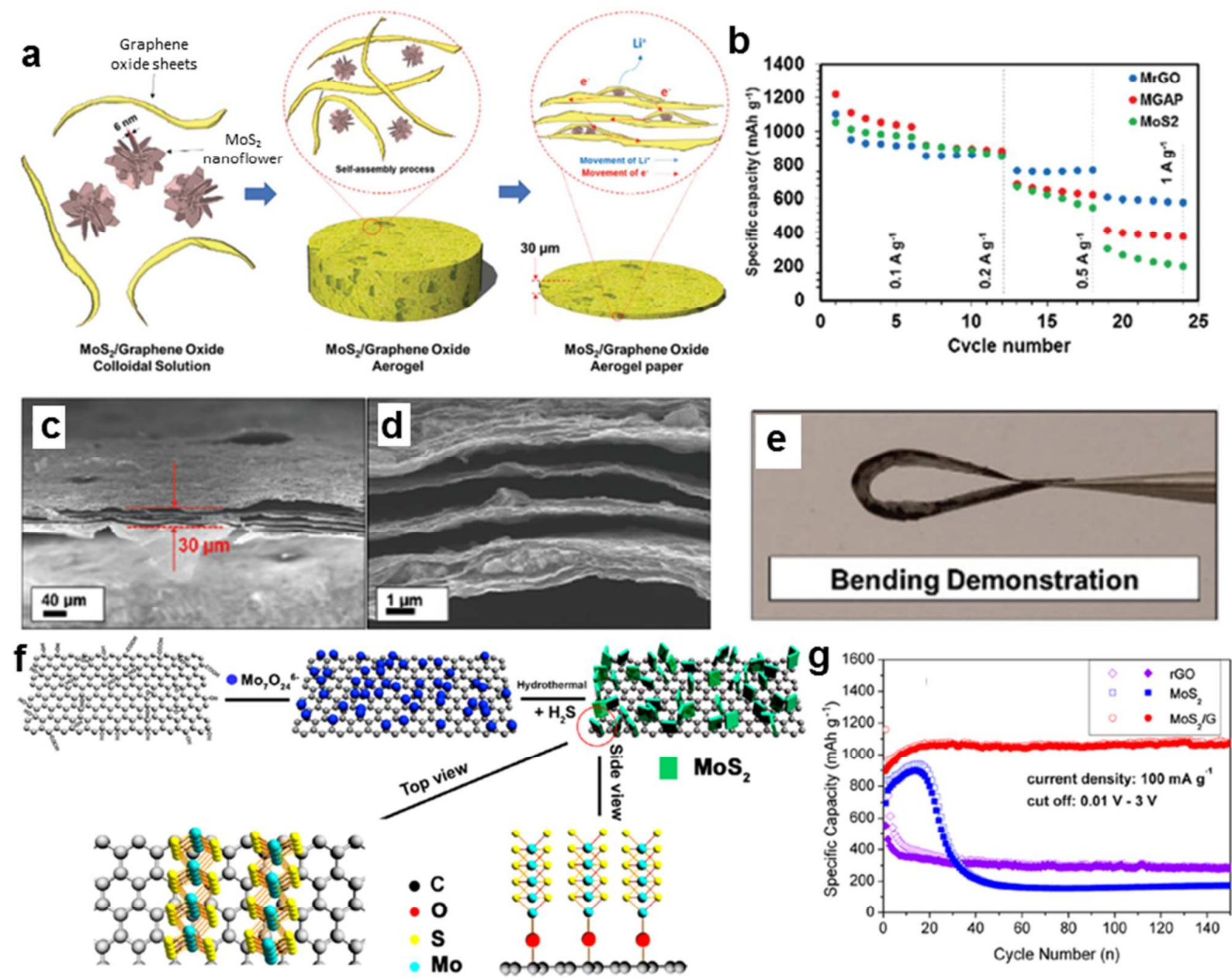

Figure 21. $\mathrm{MoS}_{2}$ /graphene hybrid composites for lithium ion batteries. (a) Schematic illustration showing the procedure for the formation of a highly porous $\mathrm{MoS}_{2} / \mathrm{GO}$ aerogel structure, along with the lithium intercalation pathways demonstrated. (b) Charge storage capacity for the single component and $\mathrm{MoS}_{2} / \mathrm{GO}$ hybrid aerogel structure with increasing current densities. (c, d) Crosssection SEM images showing the morphology of the compressed $\mathrm{MoS}_{2} / \mathrm{GO}$ aerogel electrode, illustrating the laminar structure which is ideal for ion intercalation. (e) Photograph demonstrating the flexibility of the $\mathrm{MoS}_{2} / \mathrm{GO}$ hybrid composite electrode, indicating its suitability for flexible energy storage. (f) Schematic illustration showing the synthetic procedure to form a perpendicularly aligned $\mathrm{MoS}_{2} / \mathrm{rGO}$ hybrid composite structure. (g) Comparison of the cycling performance (current density of 0.1 A. ${ }^{-1}$ ) of the single $\mathrm{MoS}_{2}$ and $\mathrm{rGO}$ electrodes to the hybrid composite material. Both single component electrodes suffer from poor stability and low capacity, while the composite has both high capacity and stability. (a-e) Modified from ref. 239 with permission from The Royal Society of Chemistry. (f-g) Adapted with permission from ref. 241. Copyright 2016 American Chemical Society. 

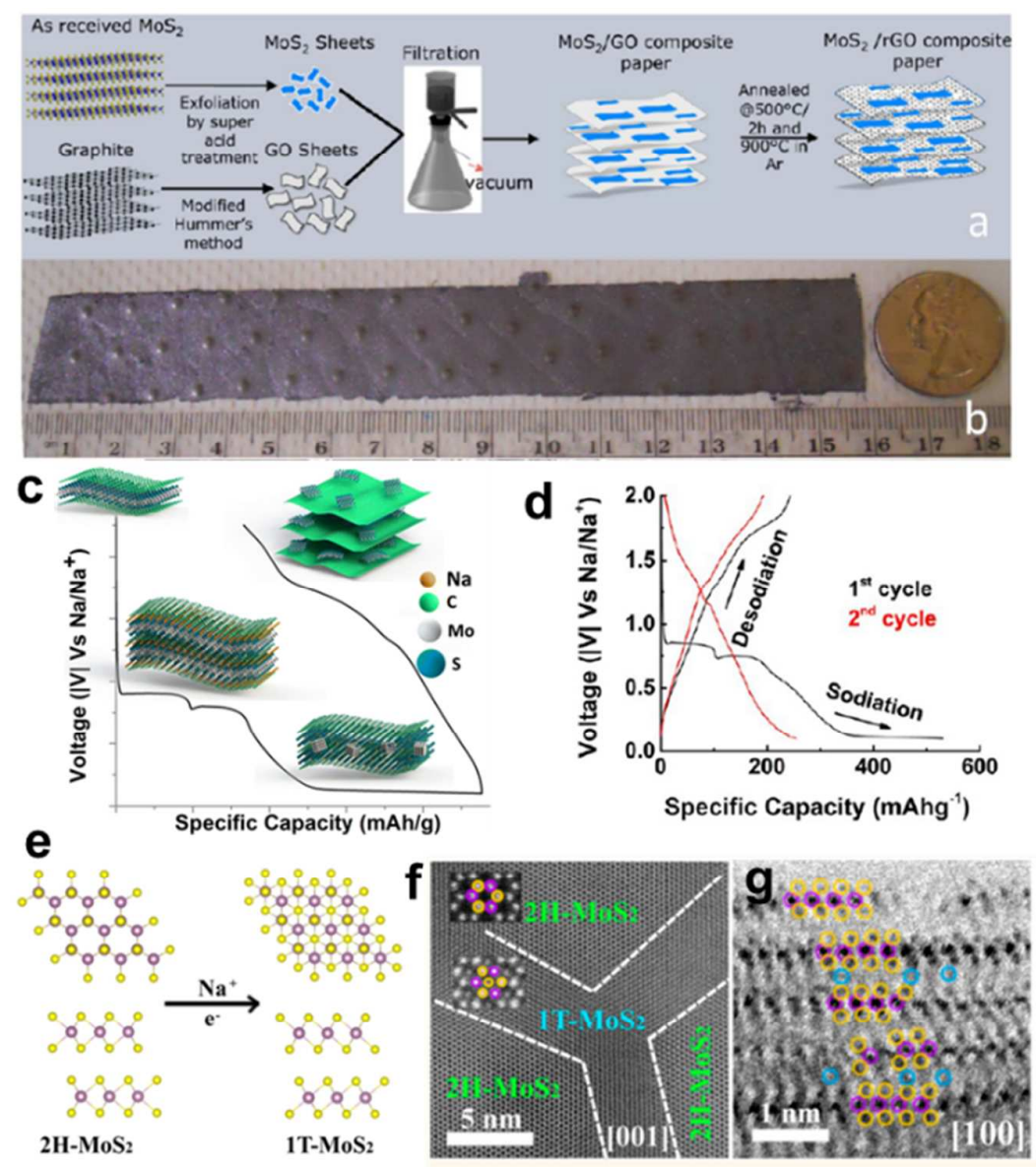

Figure 22. $\mathrm{MoS}_{2}$ /graphene hybrid composites for sodium ion batteries. (a) Schematic diagram illustrating the synthesis of $\mathrm{MoS}_{2} / \mathrm{rGO}$ composite paper. (b) Photograph of a large area composite paper electrode formed by vacuum filtration. (c) Plot demonstrating the process of intercalation and de-intercalation of sodium ions into the composite structure. (d) Graph showing the electrochemical performance of the $\mathrm{MoS}_{2} / \mathrm{rGO}\left(60 \% \mathrm{MoS}_{2}\right)$ composite for sodium intercalation/deintercalation over the first two cycles. (e) Schematic showing the phase transition that occurs in $2 \mathrm{H}-\mathrm{MoS}_{2}$ when sodium ions $\left(\mathrm{Na}^{+}\right)$are intercalated, forming $1 \mathrm{~T}-\mathrm{MoS}_{2}$. (f) High-angle annular dark-field (HAADF) image showing the different domains of $1 \mathrm{~T}$ - and $2 \mathrm{H}-\mathrm{MoS}_{2}$ formed after sodium intercalation. (g) Annular bright field (ABF) cross-section image showing the sodium ions (blue circles) intercalated between the layers of $M_{0} S_{2}$. (a-d) Adapted with permission from ref. 214 Copyright 2014 American Chemical Society. (e-g) Adapted with permission from ref. 270. Copyright 2014 American Chemical Society. 

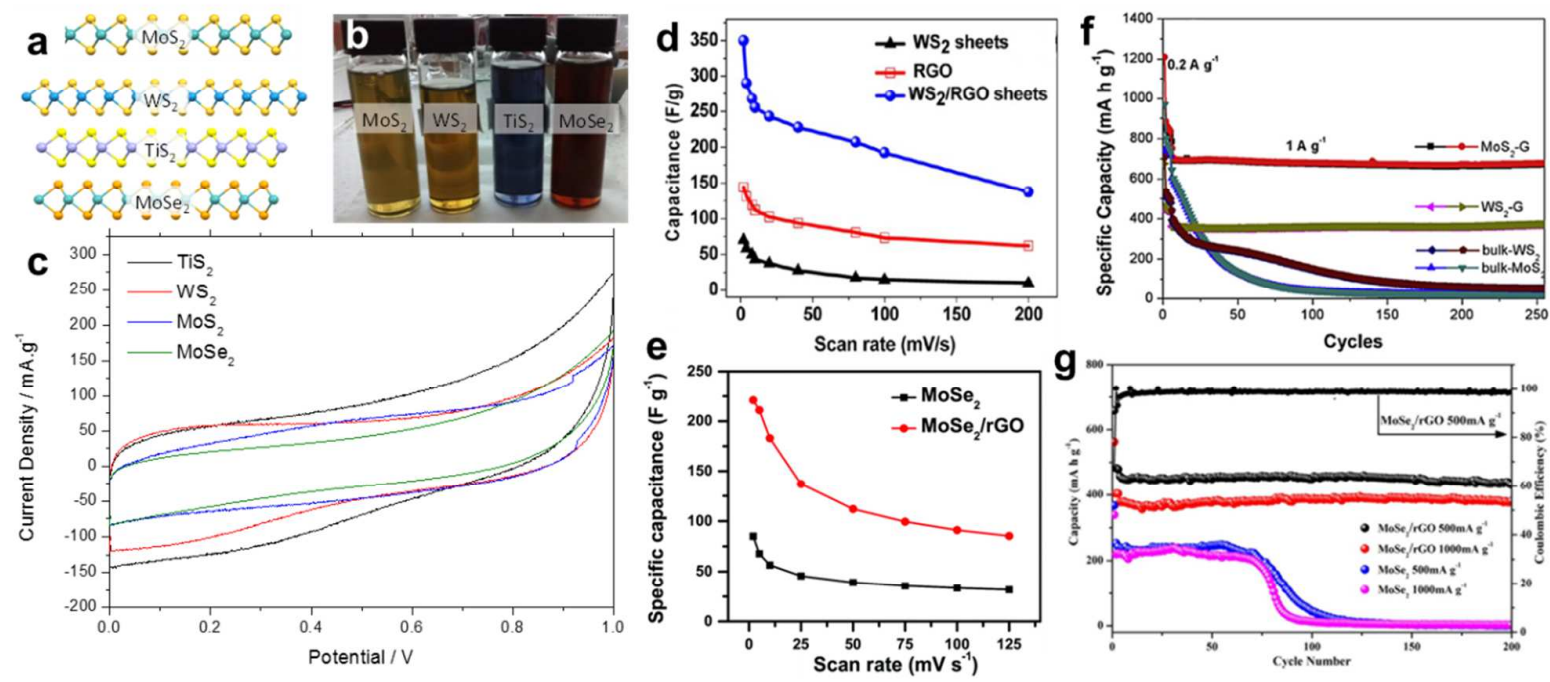

Figure 23. Hybrid composites of other TMDs. (a) Structure of the four most popular TMDs investigated for energy storage applications along with a photograph (b) of ultrasonication exfoliated dispersions of these materials. (c) Comparison of the electrochemical charge storage performance for each of these TMDs without any additives. Plots demonstrating the enhanced capacitance of a $\mathrm{WS}_{2} / \mathrm{rGO}$ (d) and $\mathrm{MoSe}_{2} / \mathrm{rGO}$ (e) hybrid composite compared to the single components. Plot showing the increased capacity and increased cycle lifetime of $\mathrm{MoS}_{2} /$ graphene and $\mathrm{WS}_{2} /$ graphene composites (f), and a $\mathrm{MoSe}_{2} / \mathrm{rGO}$ composite (g) for use in lithium ion batteries. (a-c) Adapted with permission from ref. 226. (d) Adapted with permission from ref. 218. Copyright 2013 American Chemical Society. (e) Adapted from ref. 273 with permission from The Royal Society of Chemistry. (f) Adapted from ref. 274, Copyright 2015, with permission from Elsevier. (g) Adapted from ref. 275 with permission from The Royal Society of Chemistry. 

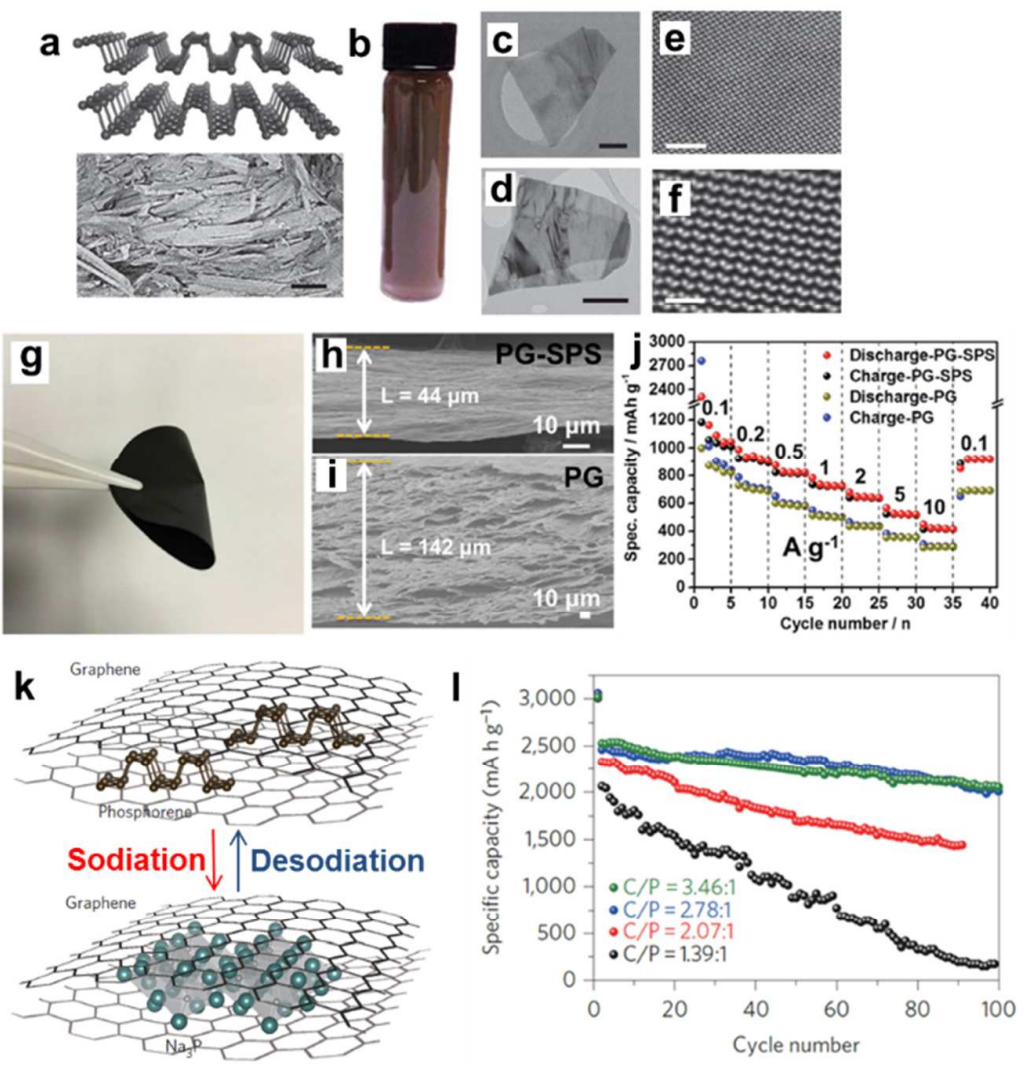

Figure 24. Phosphorene/graphene hybrid composites for energy storage. (a) Schematic and SEM image of the layered structure of black phosphorous. (b) Photograph showing a stable dispersion of ultrasonication exfoliated phosphorene. (c-f) TEM images showing the exfoliated structure and atomic lattice of exfoliated phosphorene. Scale bars $100 \mathrm{~nm}$ (c), $500 \mathrm{~nm}$ (d), $2 \mathrm{~nm}$ (e), $1 \mathrm{~nm}$ (f). (g) Photograph showing a flexible GO/phosphorene hybrid composite paper electrode. (h,i) SEM cross-section images showing the structure of the phosphorene/graphene composite (PG) paper, and that after spark plasma sintering (PG-SPS). (j) Comparison of the lithium-ion rate capability for the PG and PG-SPS composite electrodes with different current densities. (k) Schematic showing the process of sodiation/desodiation into a graphene/phosphorene composite. (I) Comparison of the charge storage capacity of hybrid composites containing different graphene (C) and phosphorene $(P)$ ratios with repeated cycling. (a-f) Adapted with permission from ref. 279. (g-j) Adapted with permission from ref. 280. Copyright 2016, John Wiley and Sons. (k,l) Adapted by permission from Macmillan Publishers Ltd: Nat. Nanotechnol. ref. 281, copyright 2015. 

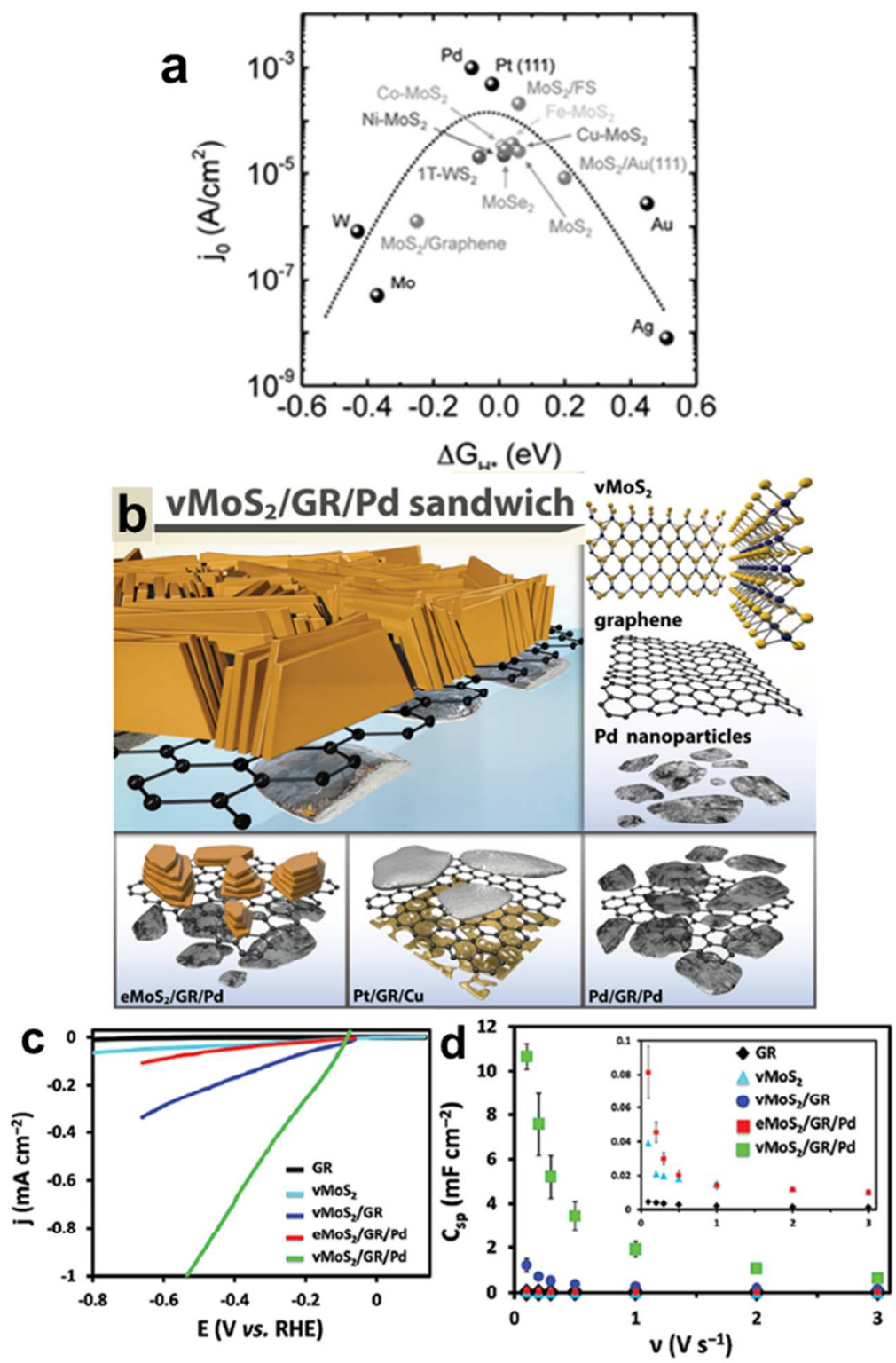

Figure 25. $\mathrm{MoS}_{2}$ /graphene composites for catalytic HER. (a) 'Volcano' plot showing the relative catalytic performance of the noble metals (Pt, Pd) compared to the TMDs $\left(\mathrm{MoS}_{2}, \mathrm{WS}_{2}\right.$ ), and hybrid composites (MoS $/$ graphene). (b) 3D illustration of the sandwich of vertically grown $\mathrm{MoS}_{2}\left(\mathrm{vMoS}_{2}\right)$, monolayer graphene and deposited metal nanoparticles. (c) Comparison of HER activity demonstrating the improved performance of the $\mathrm{vMoS}_{2} / \mathrm{Gr} / \mathrm{Pd}$ sandwich composite over the other configurations. (d) Plot comparing the capacitance of the sandwich structures after transfer to a supporting substrate, the composite $\mathrm{vMoS} / \mathrm{Gr} / \mathrm{Pd}$ structure again displays the highest capacitance and cycling stability. $\mathrm{eMoS}_{2}$ stands for liquid-phase exfoliated $\mathrm{MoS}_{2}$. (a) Reproduced with permission from ref. 282. Copyright 2016, John Wiley and Sons. (b-d) Modified with permission from ref. 287. 